\title{
Local cAMP dynamics in the SERCA2a
}

\section{signalling complex}

\author{
Doctoral Thesis
}

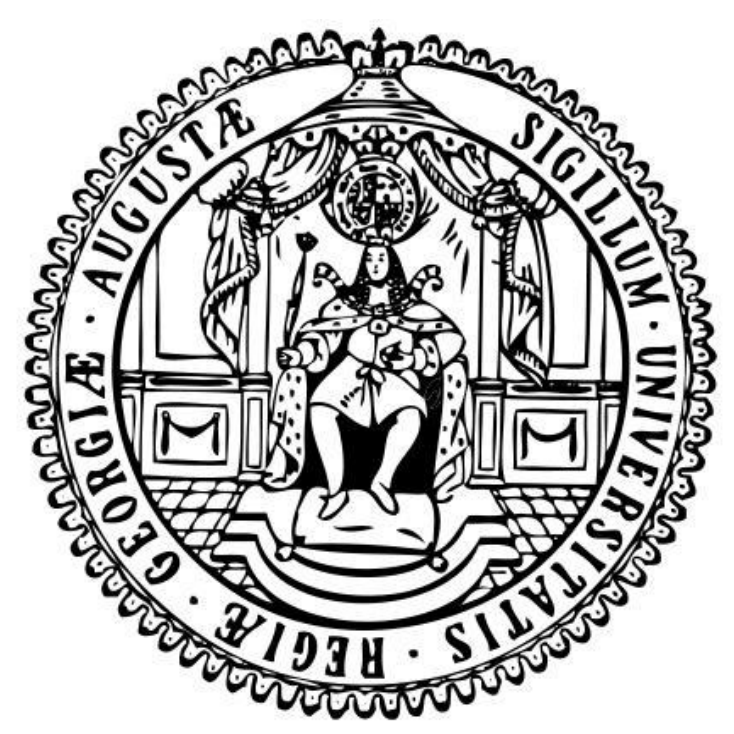

In partial fulfillment of the requirements for the degree

"Doctor rerum naturalium (Dr. rer. nat.)"

in the Molecular Medicine Study Program

at the Georg-August University Göttingen

submitted by

Dipl. Biol. Julia U. Sprenger

born in Düsseldorf, Germany

Göttingen, July 2014 


\section{Members of the Thesis Committee}

First member of the thesis committee/ Supervisor :

PD Dr. Viacheslav Nikolaev

Department of Cardiology and Pneumology

University Medical Centre, University of Göttingen

Second member of the thesis committee:

Dr. Sebastian Kügler

Department of Neurology

University Medical Centre, University of Göttingen

Third member of the thesis committee:

Prof. Dr. Peter Schu

Department of Biochemistry II

University Medical Centre, University of Göttingen

Date of Disputation: $23^{\text {rd }}$ of September 2014 


\section{Affidavit}

Here I declare that my doctoral thesis entitled

\section{"Local cAMP dynamics in the SERCA2a signalling complex"}

has been written independently with no other sources and aids than quoted.

Julia Sprenger

Göttingen, July 2014 


\section{List of Publications}

Sprenger, J.U., Perera, R.K., Gotz, K.R., and Nikolaev, V.O. (2012). FRET microscopy for real-time monitoring of signalling events in live cells using unimolecular biosensors. $\mathrm{J}$ Vis Exp, e4081.

Sprenger, J.U., and Nikolaev, V.O. (2013). Biophysical Techniques for Detection of cAMP and cGMP in Living Cells. International journal of molecular sciences 14, 8025-8046.

Gotz, K.R., Sprenger, J.U., Perera, R.K., Steinbrecher, J.H., Lehnart, S.E., Kuhn, M., Gorelik, J., Balligand, J.L., and Nikolaev, V.O. (2014). Transgenic Mice for Real-Time Visualization of cGMP in Intact Adult Cardiomyocytes. Circulation research 114, 1235-1245.

Under Revision for Nature Communications:

Sprenger, J.U., Perera, R.K., Steinbrecher, J.H., Lehnart, S.E., Maier, L.S., Hasenfuss, G., Nikolaev, V.O. (2014). In vivo model with a targeted cAMP biosensor reveals changes in receptor-microdomain communication in cardiac disease. 


\section{$\underline{\text { Table of Contents }}$}

\section{Abbreviations}

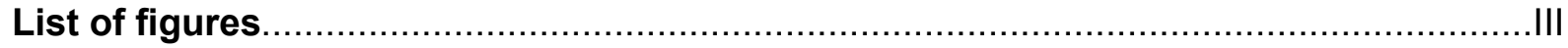

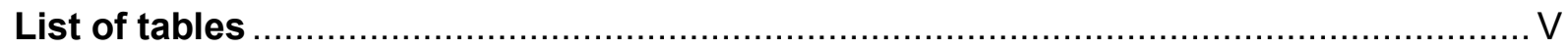

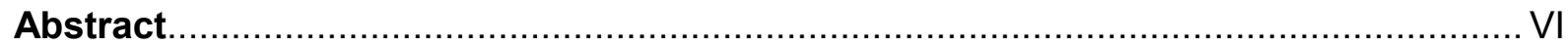

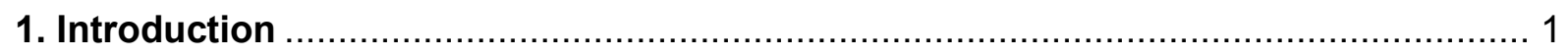

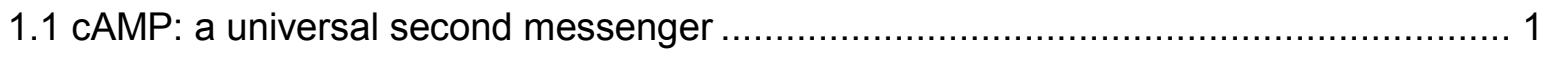

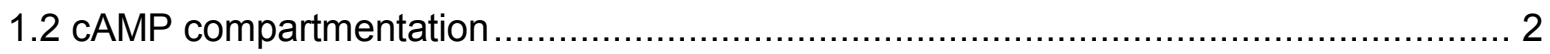

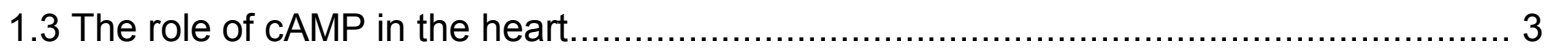

1.4 Mechanisms for cAMP compartmentation in the heart .......................................... 4

1.5 Composition and regulation of the SERCA2a microdomain ................................... 7

1.6 Changes in cAMP compartmentation during heart disease with a focus on alterations in

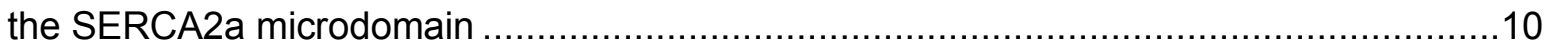

1.7 Methods for cAMP detection in subcellular microdomains ...................................13

1.7.1 Fluorescence resonance energy transfer based cAMP biosensors .......................14

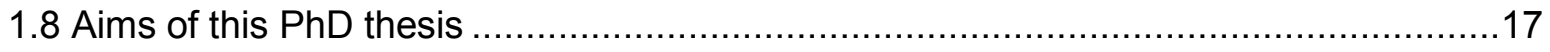

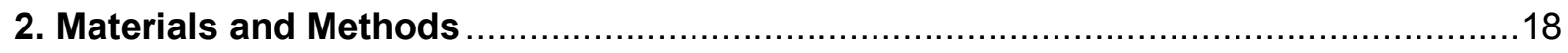

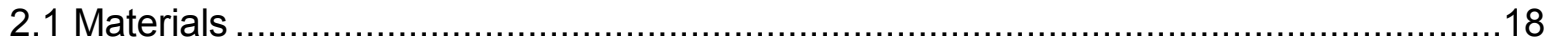

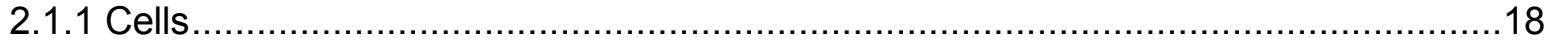

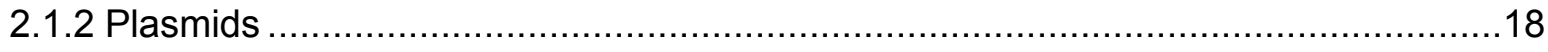

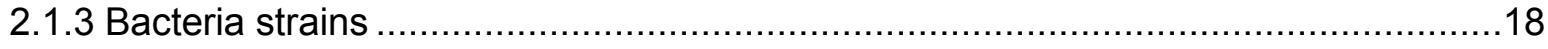

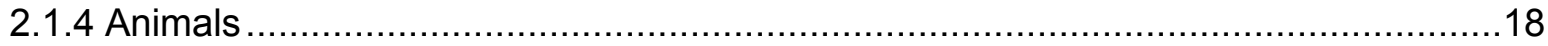

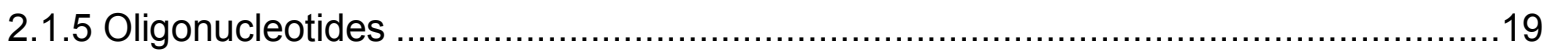

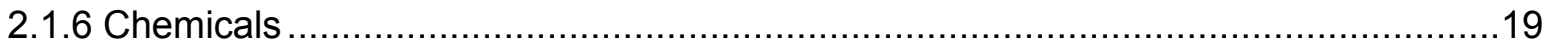

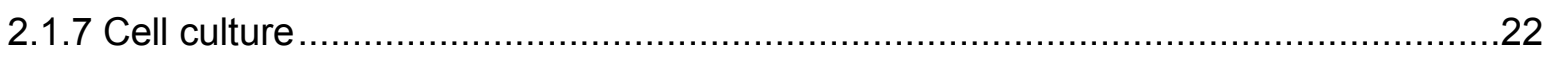

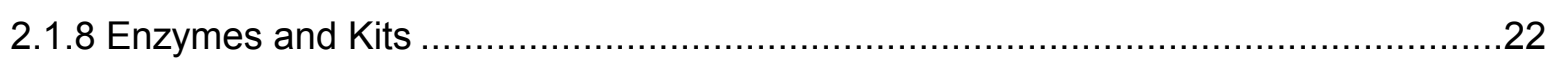

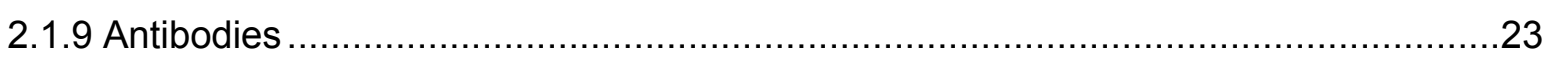

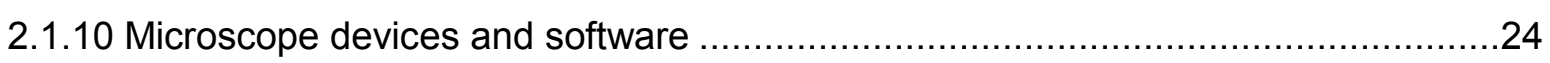

2.1.11 Calcium measurement devices and software .............................................25

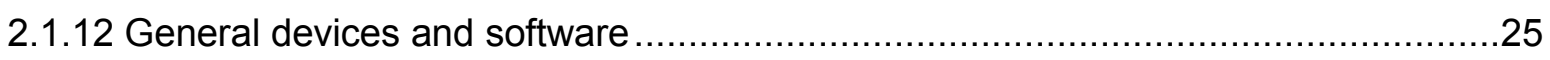

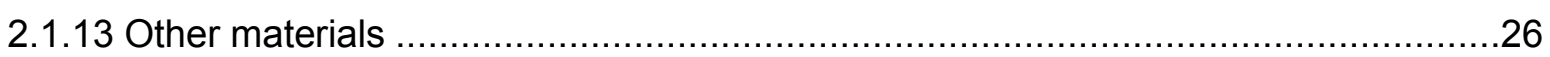

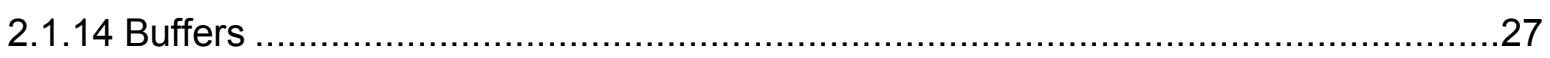

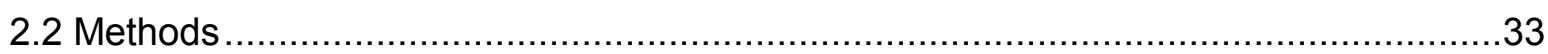




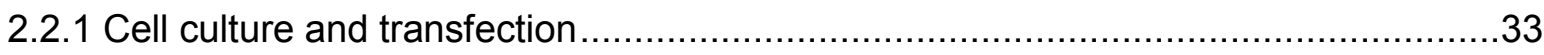

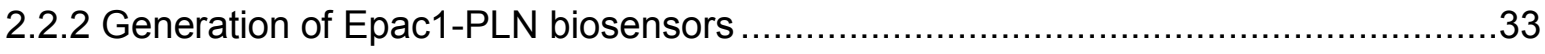

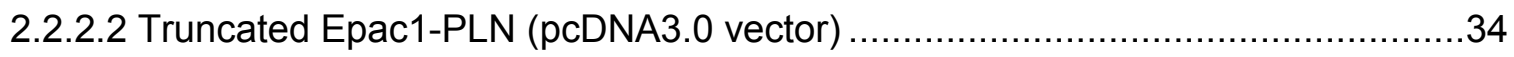

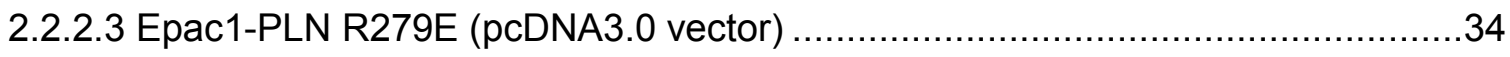

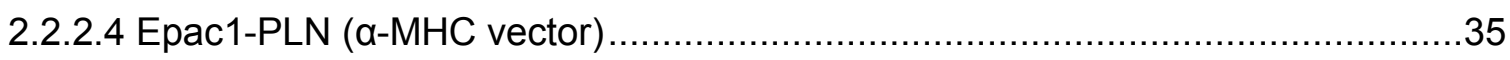

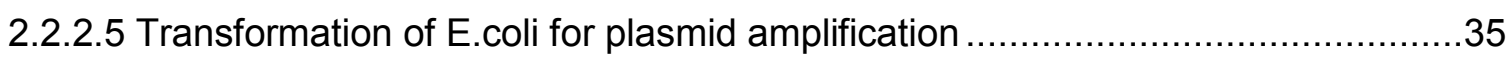

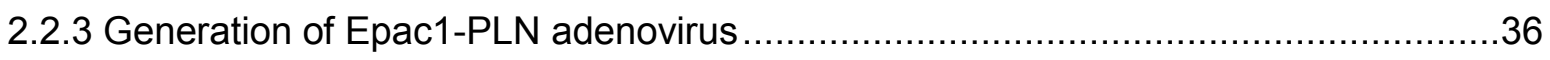

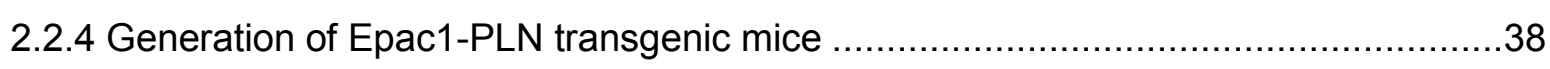

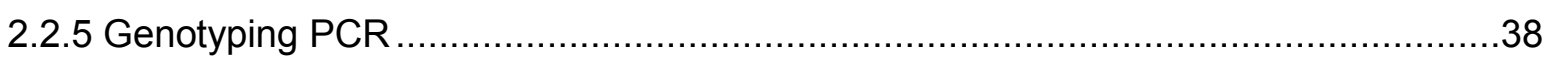

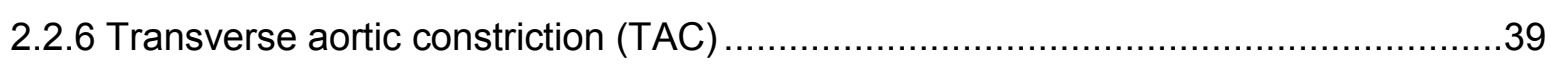

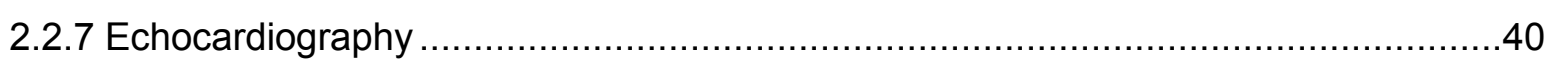

2.2.8 Heart weight to body weight and heart weight to tibia length calculation .................40

2.2.9 Cardiomyocyte isolation via Langendorff perfusion ..........................................40

2.2.10 FRET measurements in cardiomyocytes and data analysis ................................41

2.2.11 Quantitative real time PCR ..................................................................... 42

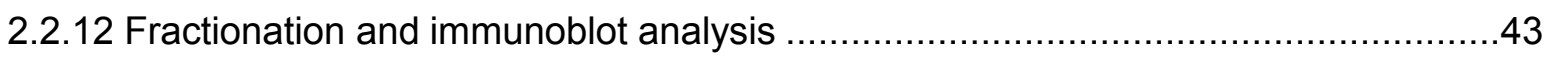

2.2.13 Histology and morphometric analysis ....................................................... 44

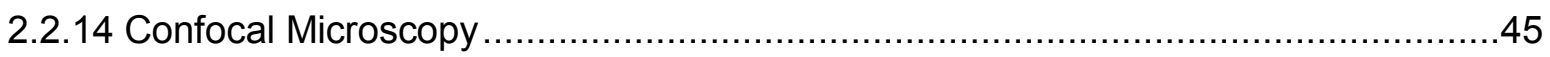

2.2.15 Calcium transients of single cardiomyocytes .............................................45

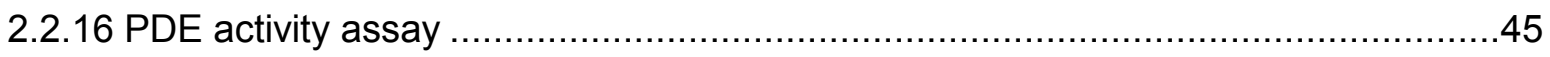

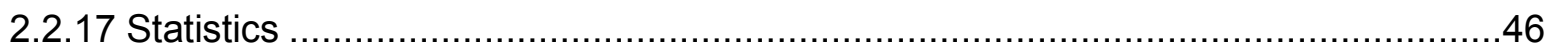

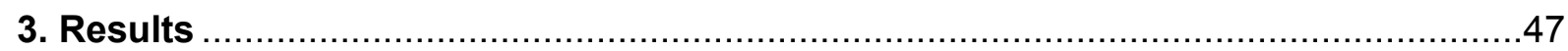

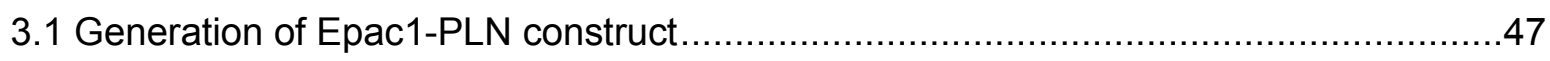

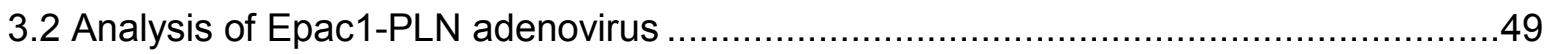

3.3 Generation and characterization of the transgenic Epac1-PLN mice ........................50

3.3.1 Generation of Epac1-PLN transgenic mice, sensor localisation and function ..........50

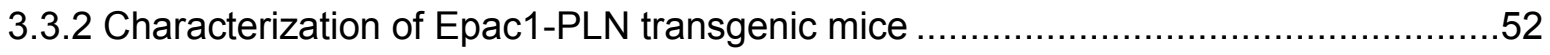

3.4 FRET measurements in Epac1-camps vs. Epac1-PLN cardiomyocytes.....................58

3.4.1 FRET measurements in Epac1-camps and Epac1-PLN cardiomyocytes reveal PDE

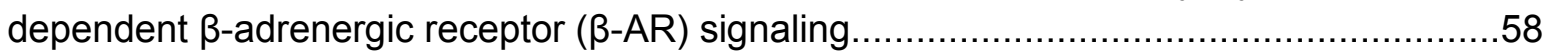

3.4.2 PDE profiles after $\beta$-adrenergic stimulation in Epac1-camps and Epac1-PLN cardiomyocytes analysed by FRET.

3.4.3 Basal PDE profiles in Epac1-camps and Epac1-PLN cardiomyocytes analysed by FRET

3.5 FRET measurements in Epac1-camps vs. Epac1-PLN cardiomyocytes after TAC surgery...... 
3.5.1 PDE contributions after TAC induced hypertrophy in the bulk cytosol vs. the SERCA2a compartment.

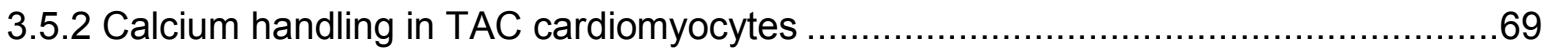

3.6 Influence of calcium on global cAMP FRET signals ............................................70

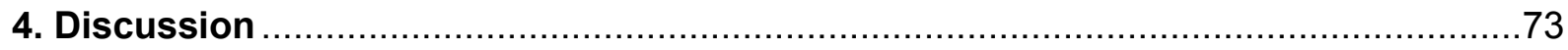

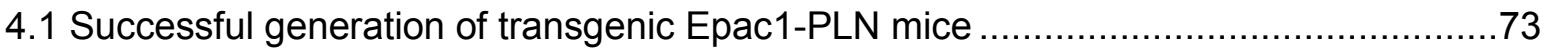

4.2 Localised FRET measurements reveal differentially regulated cAMP dynamics in healthy Epac1-camps and Epac1-PLN cardiomyocytes

4.2.1 Local PDE effects are essential for channelling cAMP from the $\beta 1-A R$ to the SERCA2a microdomain

4.2.2 PDE4 plays the predominant role in restricting cAMP signals after $\beta-A R$ stimulation and under basal conditions

4.3 Localised FRET measurements reveal differentially regulated cAMP dynamics in diseased Epac1-camps and Epac1-PLN cardiomyocytes

4.3.1 After $\beta$-AR stimulation, PDE4 and PDE2 contributions are altered in TAC treated cardiomyocytes from Epac1-camps and Epac1-PLN mice, respectively.

4.3.2 Under basal conditions, PDE4 contribution to cAMP degradation is decreased in the SERCA2a compartment after TAC surgery.

4.4 Calcium fluctuations do not influence FRET measurements via Epac1-camps .82

4.5 Summary and Conclusions 


\section{Abbreviations}

\begin{tabular}{|c|c|}
\hline AAV & Adeno-associated-virus \\
\hline$A C$ & Adenylyl cyclase \\
\hline AKAP & A kinase anchoring protein \\
\hline AWThd & Anterior wall thickness in diastole \\
\hline bpm & beats per minute \\
\hline$\beta_{1}-A R$ & $\beta_{1}$ - adrenergic receptor \\
\hline$\beta_{2}-A R$ & $\beta_{2}$ - adrenergic receptor \\
\hline BW & Body weight \\
\hline $\mathrm{Ca}^{2+}$ & Calcium \\
\hline CaMKII & $\mathrm{Ca}^{2+}$-calmodulin-dependent protein kinase \\
\hline cAMP & $3,-5,-$-cyclic adenosine monophosphate \\
\hline camps & cAMP sensor \\
\hline CFP & Cyan fluorescent protein \\
\hline cGMP & 3"-5"-cyclic guanosine monophosphate \\
\hline CNGC & Cyclic nucleotide gated channel \\
\hline CREB & cAMP response element binding protein \\
\hline ECC & Excitation contraction coupling \\
\hline EF & Ejection fraction \\
\hline Epac & Exchange protein directly activated by cAMP \\
\hline FAS & Fractional area shortening \\
\hline FRET & Förster resonance energy transfer \\
\hline FS & Fractional shortening \\
\hline fwd & forward \\
\hline $\mathrm{G}_{\mathrm{i}}$ & inhibitory G-protein \\
\hline $\mathrm{G}_{\mathrm{s}}$ & stimulatory G-protein \\
\hline GFP & Green fluorescent protein \\
\hline GPCR & G-protein coupled receptors \\
\hline HAX-1 & HS-1 associated protein X-1 \\
\hline HR & Heart rate \\
\hline HRC & Histidine-rich $\mathrm{Ca}^{2+}$ binding protein \\
\hline Hsp20 & Heat shock protein 20 \\
\hline HW & Heart weight \\
\hline $\mathrm{Hz}$ & Hertz \\
\hline $\mid-1$ & Inhibitor-1 \\
\hline $1-2$ & Inhibitor-2 \\
\hline
\end{tabular}


ISO

LTCC

LVEDD

LVESD

NCX

NRVMs

o.n.

PDE

PKA

PKC

PKG

PLM

PLN

rev

PP1

RT

RyR2

Ser

SERCA2a

SLN

SR

SUMO1

TG

Thr

Tnl

$\mathrm{V}$

WT

YFP
Isoproterenol

L-type calcium channel

Left ventricular end diastolic diameter

Left ventricular end systolic diameter

Sodium-calcium exchanger

Neonatal rat ventricular myocytes

over night

Phosphodiesterase

Protein kinase A

Protein kinase $\mathrm{C}$

cGMP dependent protein kinase

Phospholemman

Phospholamban

reverse

Phosphatase 1

Room temperature

Ryanodine receptor 2

Serine

Sarcoplasmic/endoplasmic reticulum $\mathrm{Ca}^{2+}$ ATPase 2a

Sarcolipin

Sarcoplasmic reticulum

Small ubiquitin-like modifier type 1

transgenic

Threonine

Troponin I

Volt

wildtype

Yellow fluorescent protei 


\section{List of figures}

Figure 1. Schematic representation of the cAMP signalling cascade. 2

Figure 2. Schematic representation of cardiac excitation contraction coupling and 6 the involvement of PKA within the different CAMP microdomains.

Figure 3. Regulation of SERCA2a activity via PLN modulation. 9

Figure 4. Proposed composition of the SERCA2a microdomain. 10

$\begin{array}{lll}\text { Figure 5. FRET mechanism using the CFP-YFP FRET pair. } & 14\end{array}$

Figure 6. Cartoon of the Epac1-PLN biosensor 17

Figure 7. Plasmid map of the pcDNA3.1 Epac1-PLN construct 33

Figure 8. FRET microscope setup. 42

Figure 9. FRET responses of the full length PLN and truncated Epac1-PLN sensors. 47

Figure 10. FRET responses of the Epac1-PLN under PKA inhibition and of the cAMP 48 insensitive Epac1-PLN R279E mutant.

Figure 11. The $\alpha-M H C$ Epac1-PLN construct. $\quad 49$

Figure 12. Epac1-PLN adenovirus. $\quad 49$

Figure 13. Representative Genotyping PCR. 50

Figure 14. Epac1-PLN (E1-PLN) localisation and function. 51

Figure 15. Morphometric characterization of Epac1-PLN transgenic mice. 52

Figure 16. Transgenic Epac1-PLN construct can form oligomers and becomes PKA 53 phosphorylated.

Figure 17. Basal PKA and CamKII dependent PLN phosphorylation is not altered in 54 transgenic (TG) vs. wildtype (WT) cardiomyocytes.

Figure 18. PLN and SERCA2a expression and calcium cycling. 56

Figure 19. Immunoblot analysis of wildtype (WT) vs. Epac1-PLN transgenic (TG) 57 cardiomyocytes regarding NCX and $\mathrm{Ca}_{\vee} 1.2$ expression.

Figure 20. Immunoblot analysis of wildtype (WT) vs. Epac1-PLN transgenic (TG) 57 cardiomyocytes regarding PDE expression.

Figure 21. Immunoblot analysis of wildtype (WT) vs. Epac1-PLN transgenic (TG) 58 cardiomyocytes regarding PKA expression.

Figure 22. $\beta_{1}$-AR stimulation induces stronger FRET changes in the SERCA2a 59 compartment compared to the bulk cytosol.

Figure 23. $\beta$-AR stimulation in Epac1-PLN cardiomyocytes under resting and field $\quad 60$ stimulated conditions. 
Figure 24. Sensitivity of Epac1-camps and Epac1-PLN to cAMP and increasing isoproterenol (ISO) concentrations.

Figure 25. The difference between Epac1-camps and Epac1-PLN in the magnitude of $\beta$-AR- cAMP signals is abolished after PDE pre-inhibition.

Figure 26. PDE4 plays the predominant role for cAMP degradation after $\beta$-AR stimulation in both the cytosol and the SERCA2a compartment.

Figure 27. PDE contribution to cAMP hydrolysis under basal conditions.

Figure 28. Epac1-PLN localisation is not altered in TAC mice.

Figure 29. Contributions of PDEs to cAMP hydrolysis after $\beta$-AR stimulation in sham and TAC cardiomyocytes.

Figure 30. TAC-associated changes in cAMP hydrolysis under basal conditions. 68

Figure 31. Whole-cell PDE activity and PDE protein levels are not significantly altered in TAC vs. sham mice.

Figure 32. Reduced SERCA2a expression and delayed calcium $\left(\mathrm{Ca}^{2+}\right)$ transient decay in TAC cells.

Figure 33. cAMP dynamics in adult mouse ventricular cardiomyocytes upon treatment with cAMP elevating reagents and PDE4 inhibition.

Figure 34. cAMP dynamics in adult mouse cardiomyocytes upon treatment with cAMP elevating agents and PDE1 inhibition.

Figure 35. Schematics showing proposed changes in local cAMP signalling occurring 84 in hypertrophy. 


\section{List of tables}

Table 1 Primary antibodies for Western Blot (WB) and Immunhistology (IH) 23

Table 2 Secundary antibodies for Western Blot (WB) and Immunhistology (IH) 24

Table 3 Buffer for plasmid dialysis 26

Table 4 Buffer and media for E.coli transformation 27

Table 5 Solutions for HEK293A cell transfection 27

$\begin{array}{lll}\text { Table } 6 & \text { Solutions for adenovirus purification } & 27\end{array}$

Table 7 Solutions for cardiomyocyte isolation 28

$\begin{array}{lll}\text { Table } 8 & \text { Tyrode solutions } & 29\end{array}$

Table 9 Immunoblot solutions $\quad 30$

Table 10 Solutions for PDE activity assay 32

Table 11 Echocardiographic phenotyping of the wildtype vs. Epac1-PLN transgenic 55 mice at 3 months of age.

Table 12 Echocardiographic phenotyping of wildtype, Epac1-PLN and Epac1-camps 66 transgenic mice 8 weeks after TAC vs Sham surgery. 


\section{Abstract}

3",5"-cyclic adenosine monophosphate (cAMP) is a ubiquitous second messenger that regulates multiple physiological functions by acting in distinct subcellular microdomains. Over the last few years, several targeted biosensors have been developed and used in cell lines or neonatal cardiomyocytes to investigate the molecular mechanisms behind cAMP compartmentation. However, it is unclear whether such biosensors can be successfully used for expression in vivo, especially in the context of disease such as cardiac hypertrophy.

Importantly, cAMP regulates cardiac function by acting in distinct subcellular microdomains which are independently regulated and confined from the bulk cytosol. Today, this phenomenon is a well accepted paradigm known as cAMP compartmentation. In the heart, one of these microdomains is believed to be located around the sarcoplasmic/endoplasmic reticulum calcium ATPase 2a (SERCA2a). SERCA2a is crucial for diastolic calcium $\left(\mathrm{Ca}^{2+}\right)$ reuptake and is negatively regulated by phospholamban (PLN). cAMP binding to PKA leads to increased PLN phosphorylation thereby relieving the inhibitory effect of PLN on SERCA2a. Interestingly, SERCA2a expression and activity are known to be downregulated during cardiac disease but cAMP dynamics in such microdomains and their alterations in cardiac disease such as hypertrophy are not well understood. Therefore, the first transgenic mouse model expressing a cardiac specific SERCA2a targeted fluorescence resonance energy transfer (FRET)-based cAMP sensor, namely Epac1-PLN, has been developed in this PhD study.

Freshly isolated adult cardiomyocytes of the transgenic mouse line have been used to directly monitor cAMP with high temporal and spatial resolution within the SERCA2a microdomain. To understand the molecular mechanisms that confine the SERCA2a microdomain from the bulk cytosol, FRET results gained in Epac1-PLN cardiomyocytes were compared to those obtained in cardiomyocytes expressing the cytosolic CAMP FRET sensor Epac1-camps. In healthy cells, local cAMP levels in the SERCA2a microdomain after $\beta$ adrenergic receptor $(\beta-A R)$ stimulation were $\sim 4$-fold higher compared to the bulk cytosol, which was due to direct phosphodiesterase (PDE)-dependent receptor-microdomain communication. Under basal conditions (in the absence of $\beta$-AR stimulation) PDE3 and PDE4 were crucial for confining the SERCA2a microdomain from the cytosol. However, in cardiac hypertrophy induced by transverse aortic constriction, the local basal PDE4-mediated CAMP degradation was significantly diminished, while the cytosolic cAMP dynamics were altered only after $\beta-A R$ stimulation. Strikingly, local cAMP degradation but not whole-cell changes in PDE activity in hypertrophy led to a dramatic loss of receptor-microdomain 
communication. In this study, the biocompatibility of the targeted Epac1-PLN biosensor and its potential for real-time monitoring of compartmentalized cAMP signalling in adult cardiomyocytes isolated from healthy mice and from in vivo cardiac disease model was confirmed. In particular, data show that real-time dynamics of cAMP in the SERCA2a microdomain are vastly different from bulk cytosolic cAMP due to local PDE effects and direct receptor-microdomain communication. In cardiac hypertrophy, these processes are dramatically altered which might explain impaired regulation of SERCA2a activity in disease.

$\mathrm{Ca}^{2+}$ and cAMP play a critical role for cardiac excitation-contraction-coupling and are known to interact with each other, for example via $\mathrm{Ca}^{2+}$-dependent modulation of PDE1 and adenylyl cyclases 5 and 6 activities. Currently, many FRET studies analyse cAMP signalling and its regulation in resting cardiomyocytes devoid of electrical stimulation to avoid contraction artefacts during the FRET measurements. However, it is not known how such data are comparable with the behaviour of cells under more physiologically relevant conditions during contraction. In this thesis, cAMP-FRET responses to $\beta$-AR stimulation and PDE1 inhibition were directly compared in resting vs. electrically stimulated adult mouse ventricular cardiomyocytes expressing Epac1-camps. Interestingly, no significant differences in cAMP dynamics could be detected, suggesting low impact of rapidly changing $\mathrm{Ca}^{2+}$ concentrations on cytosolic cAMP levels associated with $\beta-A R$ signalling measured with this FRET sensor. On the other hand, after direct adenylyl cyclase activation, PDE1 contribution to total PDEmediated CAMP hydrolysis increased significantly in field stimulated cardiomyocytes. This could be mimicked by pretreatment of the cells with $\mathrm{Ca}^{2+}$ elevating agents under resting conditions. However, since $\beta-A R$ stimulation reflects the more physiological situation that is used in the FRET experiments to analyse PDE contributions to cAMP hydrolysis, the use of resting cells for FRET-based cAMP measurements can be justified. 


\section{Introduction}

\section{1 cAMP: a universal second messenger}

3"-5"- cyclic adenosine monophosphate (cAMP) is a universal intracellular second messenger which regulates a large amount of different, independent cellular functions. Dependent on the cell type, these effects include for example the regulation of memory formation (Kandel, 2001; Morozov et al., 2003), insulin secretion (Holz, 2004; Leech et al., 2010; Tengholm and Gylfe, 2009), gene expression and metabolism (Altarejos and Montminy, 2011), immune reactions (Bodor et al., 2012; Brudvik and Tasken, 2012; Torgersen et al., 2002) and regulation of heart rate (Zagotta et al., 2003).

The cAMP signalling cascade starts with the extracellular activation of various G-protein coupled receptors (GPCRs) by their respective ligands. This process leads either to the activation or inhibition of cAMP-synthesizing enzymes adenylyl cyclases (ACs), via stimulatory $\left(G_{s}\right)$ or inhibitory $\left(G_{i}\right)$ G-proteins, respectively. $G_{s}$ induced AC dependent cAMP production happens within 2 seconds after receptor stimulation (Hein et al., 2006). Subsequently, cAMP activates three different downstream effectors such as cyclic nucleotide gated channels (CNGCs) (Biel et al., 1999; Craven and Zagotta, 2006), exchange protein directly activated by cAMP (Epac, isoforms are Epac1and Epac2) (Biel et al., 1999; Gloerich and Bos, 2010; Kawasaki et al., 1998) and cAMP-dependent protein kinase A (PKA) (Jurevicius and Fischmeister, 1996; Tasken and Aandahl, 2004; Taylor et al., 1990). In higher animals, PKA is a tetramer built of two regulatory (R)- and two catalytic (C)-subunits (Kim et al., 2005; Taylor et al., 1990). There are four different PKA-R isoforms (PKA-RI PKA-RI $\beta, P K A-R I_{\alpha}$ and PKA-RII ${ }_{\beta}$ ) which are all expressed in cardiomyocytes (Scholten et al., 2007). Upon cAMP binding, the C-subunits dissociate from the R-subunits (Krebs and Beavo, 1979; Taylor et al., 2008) and phosphorylate a myriad of downstream targets critical for the regulation of cellular physiology. One of these downstream targets is the transcripton factor cAMP response element- binding protein (CREB) (Altarejos and Montminy, 2011; Muller et al., 2001). In muscle cells, PKA phosphorylates several downstream effectors important for the regulation of calcium $\left(\mathrm{Ca}^{2+}\right)$ homeostasis and excitation/contraction coupling (ECC) (Lompre et al., 2010) such as troponin I (Tnl) (Bers, 2002), L-type $\mathrm{Ca}^{2+}$ channels (LTCCs) (Keef et al., 2001), the ryanodine receptor 2 (RyR2) (Takasago et al., 1989) and phospholamban (PLN) (Kirchberger et al., 1972; MacLennan and Kranias, 2003). cAMP hydrolysing enzymes phosphodiesterases (PDEs) are crucial for negatively regulating cAMP levels and therefore, to control the duration of each specific cAMP signalling event (Figure 1). 


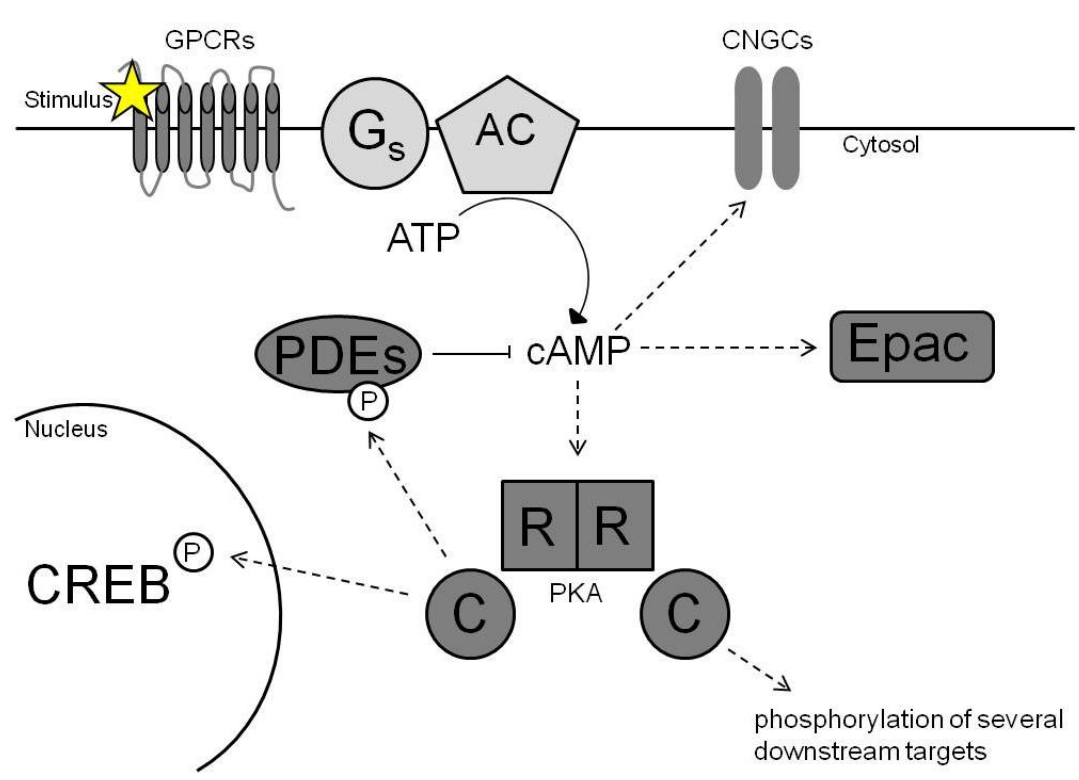

Figure 1. Schematic representation of the cAMP signalling cascade. Upon extracellular stimulation of a GPCR, CAMP production is induced via $\mathrm{G}_{\mathrm{s}}$ dependent $A C$ activation. CAMP activates three different downstream effectors: CNGCs, Epac and PKA. The catalytic (C) subunits of the PKA dissociate from the regulatory $(R)$ subunits and phosphorylate several downstream targets such as CREB and PDEs. PDEs are important for the degradation of CAMP and therefore, for the termination of the signalling cascade. Some PDEs become stimulated upon PKA dependent phosphorylation.

This whole cAMP cascade serves to amplify extracellular signals: a single GPCR, activated by nanomolar amounts of an extracellular hormone, can switch on several G-proteins, each leading to the activation of an AC and to the generation of micromolar amounts of the second messenger cAMP within a second (Lamb and Pugh, 1992). In the end of each cascade, an extracellular stimulus has been translated into an intracellular, physiological response.

\section{2 cAMP compartmentation}

Besides the different physiological effects of cAMP in different cell types, it has been shown over the last 35 years that cAMP can trigger different responses even within the same cell. The various effects depend on the nature of the extracellular stimulus and the associated receptor. For example, the $\beta$-adrenergic agonist isoproterenol (ISO) and the prostaglandin receptor agonist prostaglandin E1 both lead to an increase of cAMP within rabbit cardiomyocytes, but only ISO can trigger phosphorylation of TnI (Brunton et al., 1979; Hayes et al., 1979). Besides, ISO has been shown to stimulate cAMP in both membrane and cytosolic fractions of cardiomyocytes, whereas prostaglandin exclusively stimulated cAMP in the cytosolic fraction (Brunton et al., 1981; Buxton and Brunton, 1983). These results led to the hypothesis that cAMP signalling is organized in spatially defined subcellular 
compartments, so called microdomains, which are independent from the bulk cytosol regarding their receptor and enzyme composition or their specific regulation (Fischmeister et al., 2006; Zaccolo, 2009). Each cAMP microdomain contains a specific subset of differentially localised receptors and A-kinase anchoring proteins (AKAPs) which target protein kinases, PDEs and phosphatases in close proximity to their substrates (Diviani et al., 2011; Mauban et al., 2009; Scott et al., 2013; Troger et al., 2012). Besides AKAPs, PDEs are most important for maintaining the subcellular cAMP microdomains by shaping intracellular cAMP gradients and by confining the local cAMP pools from the bulk cytosol (Conti and Beavo, 2007; Fischmeister et al., 2006; Houslay et al., 2007).

Today, the cAMP microdomain hypothesis is a well-accepted paradigm known as cAMP compartmentation. Cardiac specific cAMP compartmentation is described in more detail in section 1.4 .

\subsection{The role of cAMP in the heart}

In the mammalian heart, cAMP primarily regulates chronotropic (beating frequency), inotropic (force of contraction) and lusitropic (force of relaxation) effects in the so called "fight or flight" response (Perera and Nikolaev, 2013). As already described in 1.1, stimulation of GPCRs such as $\beta$-adrenergic receptors ( $\beta$-ARs) via adrenaline or noradrenaline, leads to the regulation of intracellular cAMP production. There are three different types of $\beta$-ARs expressed in mammalian hearts: $\beta_{1^{-}}, \beta_{2^{-}}$and $\beta_{3}$-ARs. The $\beta_{1}$-ARs represent $70-80 \%$ of total cardiac $\beta$-ARs and couple to $G_{s}$ proteins, thereby leading to increased cAMP production. However, chronic $\beta_{1}$-adrenergic stimulation leads to congestive heart failure accompanied by a downregulation of the $\beta_{1}$ AR and a loss of cardiac pump function (Lohse et al., 2003; Michel et al., 1990). $\beta_{2}$-ARs represent $20-30 \%$ of total $\beta$-ARs and couple to both, $G_{s}-$ and $G_{i-}$ proteins.. A switch from $G_{s}$ to $G_{i}$ coupled $\beta_{2}$-AR signalling occurs upon prolonged agonist application to decrease the cAMP signal (Xiao, 2001). $\beta_{2}$-AR stimulation has been shown to have more beneficial effects on the heart as it protects from cardiac hypertrophy, heart failure or apoptosis (Communal et al., 1999) via $\mathrm{G}_{\mathrm{i}}$-mediated activation of protein kinase B (Akt) and phosphoinositide 3 kinases (Bernstein et al., 2005; Patterson et al., 2004). $\beta_{3}$-ARs represent less than $10 \%$ of total $\beta$-ARs and are associated with the $G_{i} /$ nitric oxide pathway to suppress cardiac contractile function (Lohse et al., 2003).

In the heart, increased cAMP production leads to the activation of CNGCs, Epac1 and PKA which in turn phosphorylates several downstream targets crucial for cardiac contraction. PKA downstream targets involve $\mathrm{Ca}^{2+}$ handling proteins crucial for the cardiac ECC. These proteins include LTCCs, the RyR2, contractile proteins as Tnl and PLN. PLN phosphorylation relieves its inhibition from the Sarcoplasmic/endoplasmic reticulum $\mathrm{Ca}^{2+}$ ATPase $2 \mathrm{a}$ 
(SERCA2a) $\mathrm{Ca}^{2+}$ pump (Lompre et al., 2010; Strang et al., 1994) and facilitates $\mathrm{Ca}^{2+}$ reuptake into the sarcoplasmic reticulum (SR). Besides SERCA2a, the sodium-Ca ${ }^{2+}$ exchanger (NCX) is important for the extrusion of $\mathrm{Ca}^{2+}$ from the cytosol by exchanging one $\mathrm{Ca}^{2+}$ against $3 \mathrm{Na}^{+}$(Schlotthauer and Bers, 2000). The driving force for this reaction is built up by the $\mathrm{Na}^{+} / \mathrm{K}^{+}$ATPase which is negatively regulated by phospholemman (PLM). Upon PKA phosphorylation of PLM, this inhibition is relieved (Despa et al., 2005; Fuller et al., 2004; Pavlovic et al., 2007; Silverman et al., 2005). Plasma membrane $\mathrm{Ca}^{2+}$ ATPase provides the third mechanism for cytosolic $\mathrm{Ca}^{2+}$ extrusion but with very minor contribution (Mohamed et al., 2013). For an overview of cardiac ECC and the involvement of PKA see Figure 2.

In vivo, mouse hearts show a contraction rate of 500-600 beats per minute (bpm) associated with a constant and rapid $\mathrm{Ca}^{2+}$ cycling within each cardiomyocyte during each contraction cycle. Besides its crucial role for cardiac contraction, $\mathrm{Ca}^{2+}$ is also known to dynamically regulate intracellular cAMP levels. This occurs for example via the stimulation of the $\mathrm{Ca}^{2+} /$ calmodulin-dependent PDE1 (Sonnenburg et al., 1993) or by inhibition of cardiac AC 5/6 activity (Willoughby and Cooper, 2007). However, it is still unclear whether or not fluctuations in cytosolic $\mathrm{Ca}^{2+}$ levels during contraction cycles may directly affect intracellular cAMP levels.

In addition to $\beta$-ARs, other cardiac receptors involved in cAMP signalling include the prostaglandin and glucagon receptors, whose cAMP pools are not associated with the regulation of cardiac contractility (Buxton and Brunton, 1983; Di Benedetto et al., 2008; Vila Petroff et al., 2001).

\subsection{Mechanisms for cAMP compartmentation in the heart}

In cardiomyocytes, several cAMP microdomains have been proposed which are located, for example, around $\mathrm{Ca}^{2+}$ handling proteins such as LTCCs, RyR2 and SERCA2a (Figure 2) (Fischmeister et al., 2006; Lompre et al., 2010). The intracellular regulation of these $\mathrm{Ca}^{2+}$ handling proteins is crucial for proper cardiac contraction and function. Therefore, alterations in CAMP microdomain regulation and organization might be associated with cardiac disease such as hypertrophy which could ultimately lead to heart failure.

PDEs are critical for the maintenance of subcellular microdomains by shaping intracellular cAMP gradients and restricting local cAMP pools within the cell. At least five families of cAMP hydrolysing enzymes PDEs (PDE1-4 and 8) are expressed in mammalian cardiomyocytes (Conti and Beavo, 2007; Fischmeister et al., 2006; Houslay et al., 2007; Zaccolo and Movsesian, 2007). PDE1, 2 and 3 are dual specific for cAMP and 3 "-5"-cyclic guanosine monophosphate (cGMP), whereas PDE4 and 8 hydrolyse cAMP exclusively. PDE activity can be regulated by several molecular mechanisms. For example, PDE1 plays an 
important role in the cAMP/Ca ${ }^{2+}$ crosstalk (Miller and Yan, 2010) as it can be activated by $\mathrm{Ca}^{2+} /$ calmodulin (Sonnenburg et al., 1993). PDE2 is a homodimer and its cAMP hydrolysing activity increases upon cGMP binding to the enzyme"s GAF domains via an allosteric mechanism (Martinez et al., 2002). In contrast, the PDE3 family (PDE3A and PDE3B subfamilies) gets inhibited by CGMP and therefore, is believed to play an important role in the regulation of cAMP/cGMP crosstalk (Stangherlin et al., 2011). The PDE3A subfamily is proposed to be the most important cAMP- degrading PDE in human cardiomyocytes and the second most important in rodent cardiomyocytes (Abi-Gerges et al., 2009; Molina et al., 2012; Weishaar et al., 1987). However, PDE4 is the predominant CAMP- degrading enzyme in mouse cardiomyocytes (Leroy et al., 2008). Four genes (PDE4A-D), which encode 20 isoforms, have been described in the mammalian system. PDE4A, $B$ and $D$ have been shown to be expressed in human and rodent hearts (Kostic et al., 1997; Richter et al., 2011). Due to their unique $\mathrm{N}$-terminal regions, these different isoforms show specific subcellular localisation (Houslay and Adams, 2003) making them crucial for cAMP compartmentation within cardiomyocytes. For example, PDE4D8 has been shown to directly interact with $\beta_{1}$ ARs via the receptor"s C-terminus, thereby regulating cAMP concentrations within this microdomain under basal conditions (Richter et al., 2008). On the other hand, PDE4D8 dissociates from the complex upon $\beta_{1}$-AR stimulation (De Arcangelis et al., 2010; Richter et al., 2008). PDE4D5, together with $\beta$-arrestin, has been shown to be recruited to the $\beta_{2}-A R$ upon receptor stimulation. This leads to enhanced PDE associated CAMP degradation and a switch from $G_{s}$ to $G_{i}$ coupled signalling via $\beta$-arrestin (Baillie et al., 2003; Perry et al., 2002; Richter et al., 2008). Furthermore, PDE4 activity can be stimulated by PKA dependent phosphorylation, leading to a negative feedback loop of cAMP regulation (MacKenzie et al., 2002).

The second cAMP specific PDE is the PDE8 family which is encoded by two genes (PDE8A$B$ ) of which only PDE8A has been shown to be expressed in the mouse and human heart (Soderling et al., 1998). Similar to PDE4, PDE8 can be phosphorylated and activated by PKA (Brown et al., 2012).

As already mentioned in section 1.2, AKAPs are crucial for building up functional cAMP microdomains. They localise to subcellular membrane locations for example due to electrostatic attraction of positively charged amino acids to negatively charged membrane lipids (Horner et al., 2012) to bring together PKA, other kinases, PDEs and phosphates (Diviani et al., 2011; Mauban et al., 2009; Scott et al., 2013; Troger et al., 2012) (Figure 2). It is believed that AKAPs anchor PKA due to a tight protein-protein interaction between an amphipathic helix and the dimerization and docking domain of the PKA-R subunit (Wong and Scott, 2004). Besides, PKA dependent autophosphorylation of the PKA-R subunit enhances the interaction between AKAPs and PKA (Zakhary et al., 2000a). However, different PKA-R 
isoforms have been shown to locate in different subcellular regions with PKA-RI mainly located in the cytosol and PKA-RII in the particulate fraction (Brunton et al., 1981; Corbin et al., 1977). It has been described that this compartment specific localisation is mediated via interaction of PKA-R subunits with different endogenous AKAPs, thereby creating distinct cAMP compartments that are controlled by specific PDE subsets (Di Benedetto et al., 2008). For example, AKAP79 interacts with the LTCC via a LTCC leucine zipper motif (Gao et al., 1997) and mediates anchoring of PKA and $A C 5 / 6$ in caveolin-3 rich membrane compartments close to the LTCC (Nichols et al., 2010). Here, PKA phosphorylates the LTCC which leads to increased $\mathrm{Ca}^{2+}$ influx after $\beta$.AR stimulation. PDE4B has been shown to restrict cAMP signals in this microdomain, thereby regulating PKA dependent LTCC phosphorylation (Leroy et al., 2011). Recently, experiments in cardiomyocytes of PDE8A knockout mice revealed an involvement of PDE8A in the regulation of $\mathrm{Ca}^{2+}$ homeostasis associated with the LTCC (Patrucco et al., 2010). Besides, AKAP18 $\alpha$ acts together with AKAP79 to regulate $\beta$ AR dependent $\mathrm{Ca}^{2+}$ transients (Hulme et al., 2003; Scott and Santana, 2010).

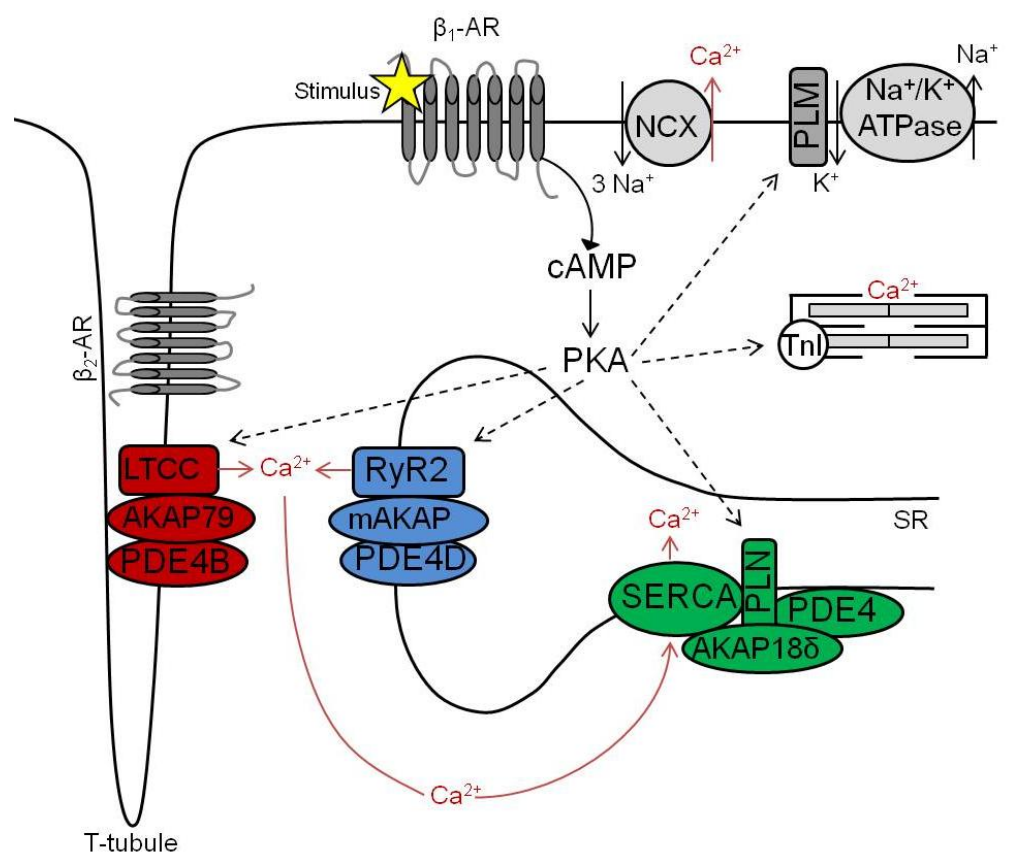

Figure 2. Schematic representation of cardiac excitation contraction coupling and the involvement of PKA within the different cAMP microdomains. With each action potential, voltage gated LTCCs open and $\mathrm{Ca}^{2+}$ flows into the cell, thereby facilitating $\mathrm{Ca}^{2+}$ release from the RyR2 (called Ca ${ }^{2+}$ induced $\mathrm{Ca}^{2+}$ release (Fabiato and Fabiato, 1977) ). $\mathrm{Ca}^{2+}$ then binds for example to $\mathrm{Tnl}$ to increase the sensitivity of the myofilaments to $\mathrm{Ca}^{2+}$. SERCA2a and NCX are most important for the removal of intracellular $\mathrm{Ca}^{2+}$. Upon $\beta_{1}$-AR stimulation, cAMP gets produced and activates PKA. PKA phosphorylates several downstream targets important for $\mathrm{Ca}^{2+}$ handling such as LTCC, RyR2, PLN, PLM and Tnl, thereby increasing chronotropy, inotropy and lusitropy. The different $\mathrm{Ca}^{2+}$ handling proteins are part of so called cAMP microdomains, which also contain different pools of PKA (not shown here for simplicity) 
It has been shown that mAKAP is responsible for targeting PKA and PDE4D3 to the RyR2 microdomain in close proximity to the protein phosphatases $1 / 2$ and calstabin. This whole complex is crucial for the regulation of $\mathrm{Ca}^{2+}$ release from the $\mathrm{SR}$ during each contraction of the heart (Dodge et al., 2001; Marx et al., 2000). The microdomain associated PDE4D3 is important for the control of local cAMP levels thereby preventing excessive RyR2 phosphorylation that else would be associated with arrhythmia and heart failure (Lehnart et al., 2005). Besides, experiments in PDE8A knockout cardiomyocytes showed a leaky RyR2 phenotype (Patrucco et al., 2010), similar to that described in PDE4D deficient mice (Lehnart et al., 2005).

The SERCA2a microdomain was the object of extensive studies within this PhD thesis and will be described in more detail in the next section.

\subsection{Composition and regulation of the SERCA2a microdomain}

The SR is the $\mathrm{Ca}^{2+}$ storage in cardiomyocytes and is crucial for the regulation of intracellular $\mathrm{Ca}^{2+}$ concentrations during ECC and therefore, contractile function. After each contraction cycle, $\mathrm{Ca}^{2+}$ is extruded from the cytosol and pumped back into the SR by SERCA2a, so that it would be available for the next contraction round.

The SERCA family consists of three gene products (ATP2A1-3) each giving rise to at least two differentially spliced isoforms. In the heart, SERCA2a isoform is the most abundant protein in the SR (Anger et al., 1994; Lompre et al., 1994; Lompre et al., 1991) and is responsible for the extrusion of $90-95 \%$ of $\mathrm{Ca}^{2+}$ during diastole in mouse and rat ventricular cardiomyocytes (Bers, 2002). SERCA2a, a P-type ATPase of 110 kDa (MacLennan, 1970), is activated by cytosolic $\mathrm{Ca}^{2+}$ concentrations greater than $100 \mathrm{nM}$ and pumps $\mathrm{Ca}^{2+}$ back into the SR against a concentration gradient at the expense of ATP hydrolysis (Arai, 2000).

PLN, a small transmembrane protein in the SR consisting of 52 amino acids (Zamoon et al., 2003), is known to be the negative regulator of SERCA2a (Limas et al., 1987). It does so by decreasing SERCA2a"s apparent $\mathrm{Ca}^{2+}$ affinity (James et al., 1989; Kim et al., 1990). Under basal conditions, $\mathrm{Ca}^{2+}$ binding to SERCA2a leads to a dissociation of the SERCA2a/PLN complex, possibly due to a $\mathrm{Ca}^{2+}$ induced conformational change within SERCA2a (Asahi et al., 2003). In addition to that, PLN activity in vivo is critically regulated by the phosphorylation of two residues, the PKA site Serine-16 (Ser-16) and the $\mathrm{Ca}^{2+}$-calmodulin-dependent protein kinase (CaMKII) site Threonine-17 (Thr-17) (Movsesian et al., 1984; Simmerman et al., 1986), thereby relieving PLN inhibition on SERCA2a and leading to enhanced SR $\mathrm{Ca}^{2+}$ transport (Inui et al., 1986; Tada and Katz, 1982). Upon catecholaminergic stimulation of $\beta_{1^{-}}$ ARs, PLN gets phosphorylated at both sites, whereby Thr-17 phosphorylation has been shown to depend on the intracellular increase of cAMP that leads to a rapid rise of 
intracellular $\mathrm{Ca}^{2+}$ which is necessary for CamKIl activation (Kuschel et al., 1999a; Lindemann et al., 1983; Lindemann and Watanabe, 1985; Mundina-Weilenmann et al., 1996; Vittone et al., 1990). However, Ser-16 phosphorylation upon $\beta_{1}$-AR stimulation is sufficient enough for relieving PLN dependent inhibition of SERCA2a and for mediating maximal cardiac response (Chu et al., 2000). Therefore, the physiological importance of Thr-17 phosphorylation is still controversial (Mattiazzi et al., 2005). In vitro experiments could show additional phosphorylation of PLN at Ser-10 by protein kinase C (PKC) and at Ser-16 by cGMPdependent protein kinase (PKG) (Frantz et al., 2013). PLN is supposed to be in a dynamic equilibrium between a monomeric and a pentameric form in which the monomer is believed to be the active, SERCA2a inhibiting form, and the pentamer the inactive storage form (Cornea et al., 1997; MacLennan and Kranias, 2003; Reddy et al., 1999). Upon phosphorylation, PLN has been believed to dissociate from SERCA2a, thereby relieving its inhibitory effect (Chen et al., 2010; James et al., 1989; Mueller et al., 2004). However, other studies showed that PLN pentamers are also able to inhibit SERCA2a activity (Zhai et al., 2000). Interestingly, latest studies on the interaction between SERCA2a and PLN showed that SERCA2a binds to completely phosphorylated PLN (Dong and Thomas, 2014) and that PLN exists in a conformational equilibrium between different intramolecular states which are phosphorylation dependent and differ in their SERCA2a inhibition characteristics (Gustavsson et al., 2013). Therefore, SERCA2a inhibition might be mediated by PLNphosphorylation induced conformational changes within the SRECA2a-PLN complex rather than by dissociation of the complex.

Furthermore, latest studies in Epac1 knockout mice identified Epac1 as an important regulator of PKA-independent PLN phosphorylation via a phospholipase $\mathrm{C} /$ protein kinase $\mathrm{C}$ pathway, thereby mediating cardiac responses to stress (Okumura et al., 2014). PLN mainly gets dephosphorylated by phosphatase 1 (PP1), thus restoring its SERCA2a inhibiting nature (MacDougall et al., 1991; Steenaart et al., 1992). PP-1 itself is regulated by its endogenous inhibitors, inhibitor-1 (I-1) and inhibitor-2 (I-2). Upon $\beta_{1}$-adrenergic stimulation, I-1 gets PKA phosphorylated at Thr-35. In cardiomyocytes that overexpress I-1 adenovirally, this Thr-35 phosphorylation led to I-1 induced PP-1 inhibition and sustained PLN-phosphorylation at Ser-16, which ultimately led to enhanced SERCA2a activity (EIArmouche et al., 2003). Sarcolipin (SLN), a small (31 amino acids) PLN homologue (Hellstern et al., 2001), regulates SERCA2a activity in a similar mechanism as PLN and has been originally co-purified with SERCA1a from skeletal muscle (Odermatt et al., 1998). In the heart, SLN is mainly expressed in the atria but significantly less in ventricular cardiomyocytes (Babu et al., 2007; Minamisawa et al., 2003). As this PhD thesis discusses the SERCA2a regulation within ventricular cardiomyocytes, there is no detailed description of the SLNSERCA2a interaction given at this point. 
Another protein located within the SERCA2a microdomain is the small heat shock protein 20 (Hsp20). It has been shown to have cardioprotective effects (Fan et al., 2005; Fan et al., 2006) and is the only small Hsp containing a consensus motif for PKA/PKG dependent phosphorylation (Chu et al., 2004). Indeed, sustained $\beta$-adrenergic stimulation induces PKA dependent Hsp20 phosphorylation at its Ser-16 site (Qian et al., 2009). This leads to a direct interaction between Hsp20 and PP-1, thereby inhibiting PP-1 enzymatic activity (Qian et al., 2011) and increasing SERCA2a activity as described for I-1 induced PP-1 inhibition (Figure $3)$.

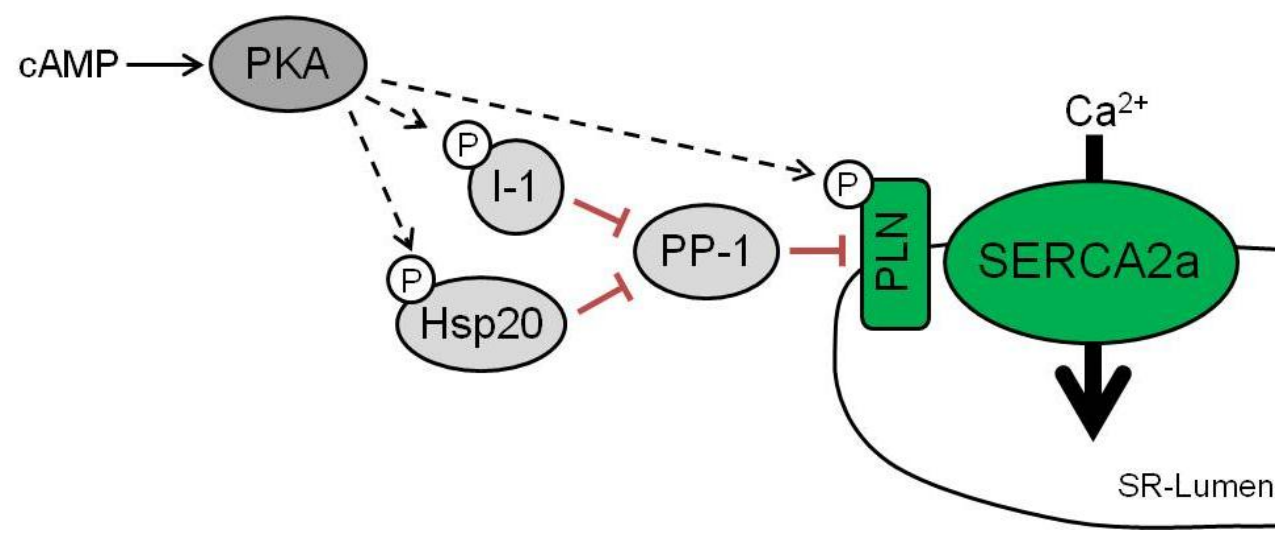

Figure 3. Regulation of SERCA2a activity via PLN modulation. CAMP leads to the activation of PKA, hereupon phosphorylating PLN (at Ser-16), I-1 (at Thr-35) and Hsp20 (at Ser-16). Phosphorylation of $\mathrm{I}-1$ and Hsp20 leads to inhibition of PP-1, thereby preventing PLN dephosphorylation. SERCA2a activity is enhanced and $\mathrm{Ca}^{2+}$ is pumped from the cytosol back to the SR lumen.

Recently, it has been shown that the HS-1 associated protein X-1 (HAX-1), a mitochondrial protein with anti-apoptotic function (Han et al., 2006), localises to the SR via direct interaction with PLN. The interaction occurs in the PLN region including the regulatory sites Ser-16 and Thr-17 and is diminished upon PLN phosphorylation by PKA or CaMKII. Hence, HAX-1 is believed to regulate PLN function in the heart (Vafiadaki et al., 2007). Indeed, binding of HAX-1 to non-phosphorylated PLN seems to increase PLN monomer formation, thereby having an inhibitory effect on SR $\mathrm{Ca}^{2+}$ reuptake by SERCA2a (Zhao et al., 2009). Besides, HAX-1 has been shown to directly interact with SERCA2a and to modulate its protein levels to promote cell survival (Vafiadaki et al., 2009).

Another protein involved in the SERCA2a microdomain regulation is a low affinity $\mathrm{Ca}^{2+}$ binding protein within the SR lumen, namely the histidine-rich $\mathrm{Ca}^{2+}$ binding protein (HRC). HRC regulates SERCA2a function in a $\mathrm{Ca}^{2+}$ dependent manner (Arvanitis et al., 2007), and direct interaction between HRC and SERCA2a inhibits SR $\mathrm{Ca}^{2+}$ uptake (Gregory et al., 2006). 
Moreover, it has been shown that that SERCA2a lifetime and activity could be prolonged and increased due to reversible covalent linking of a cytosolic protein called small ubiquitin related modifier-1 (SUMO1) in a process called sumoylation (Kho et al., 2011). This is possibly another essential mechanism for the regulation and maintenance of the SERCA2a microdomain.

PDEs are crucial for creating local cAMP pools, and different PDEs are supposed to restrict cAMP within the SERCA2a microdomain. For example, studies in PDE4D ${ }^{-1-}$ mice could detect PDE4D as a critical regulator of baseline $\mathrm{SR} \mathrm{Ca}^{2+}$ release associated with increased PLN phosphorylation (Beca et al., 2011). Besides, PDE3A has been shown to interact with SERCA2a, thereby regulating basal myocardial contractility in studies using PDE $3 A^{-/-}$and PDE3B $^{-/-}$mice (Beca et al., 2013).

As already mentioned, AKAPs are most important for the generation and maintenance of cAMP microdomains. For the association of the SERCA2a microdomain, AKAP18ס has been shown to play a critical part as it enables PKA dependent phosphorylation of PLN, thereby enhancing $\mathrm{Ca}^{2+}$ reuptake into the SR (Lygren et al., 2007). In addition, AKAP18ס has been shown to associate with $\mathrm{I}-1$ and PP-1, bringing them in close proximity to allow direct regulation of PLN function (Singh et al., 2011) .

For a summary of SERCA2a microdomain composition see Figure 4.

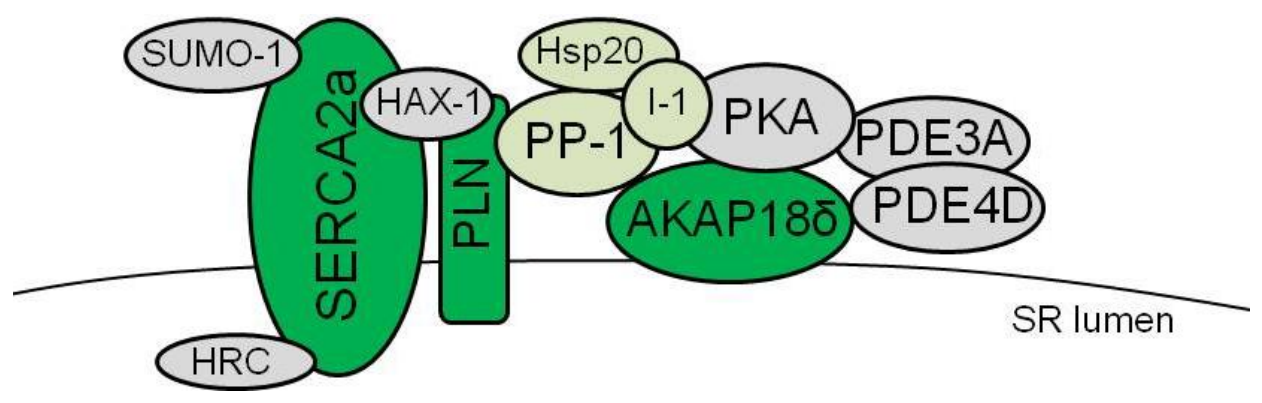

Figure 4. Proposed composition of the SERCA2a microdomain. SERCA2a activity is regulated by PLN phosphorylation status and interaction with SUMO-1, HRC and HAX-1 proteins. PLN gets dephosphorylated by PP-1 which in turn is inhibited by $\mathrm{I}-1$ and Hsp20. PDE3A and PDE4D subfamilies have been shown to be associated with this microdomain. AKAP18ס mediates the localisation of SERCA2a microdomain associated proteins such as PKA, PP-1 and I-1.

\subsection{Changes in CAMP compartmentation during heart disease with a focus on alterations in the SERCA2a microdomain.}

Today, cardiovascular diseases are the leading cause of mortality worldwide (Global status report on noncommunicable diseases 2010. Geneva, World Health Organization, 2011). For example, pathological cardiac hypertrophy is the main adaptive response to hypertension 
(Brilla et al., 1990; Devereux et al., 1987). It is characterized by cardiac remodelling which leads to the progression of compensated to decompensated hypertrophy and heart failure (Ganau et al., 1992). Compensated hypertrophy is characterized by a significant increase in wall thickness due to cardiomyocyte enlargement associated by increased cardiomyocyte protein expression and assemby of additional sarcomeric units (Bernardo et al., 2010). At this point, cardiac function is mostly preserved. On the other hand, progression to decompensated hypertrophy is associated with a drop in cardiac performance provoked by left ventricular dilatation due to cardiomyocyte apoptosis, necrosis and increased fibrosis (Diwan and Dorn, 2007). It is accompanied by reduced contractility, which, if the heart fails to provide proper blood flow to fulfil the physiological needs of the body, finally leads to heart failure (Levy et al., 1996; Rosca et al., 2013). On the molecular level, heart failure is associated with a desensitization and decreased expression of $\beta_{1}$-ARs which impairs cardiac performance even more (Bristow et al., 1982; Ungerer et al., 1993).

During cardiac disease, many changes in cAMP compartmentation have been described. In general, disrupted interaction between AKAPs and their binding partners leads to the collapse of important microdomains in diseased cardiomyocytes.

For example, PKA interaction with AKAPs is significantly decreased in the diseased human heart due to decreased PKA-R subunit phosphorylation (Zakhary et al., 2000b) which leads to decreased phosphorylation of proteins involved in cardiac $\mathrm{Ca}^{2+}$ handling. However, a disruption of the mAKAP/RyR2 complex has been reported caused by a distinct dissociation of calstabin from this complex due to RyR2 hyperphosphorylation (Huang et al., 2006; Marx et al., 2000; Shan et al., 2010). This PKA dependent hyperphosphorylation might result from a PDE4D depletion from the RyR2 complex under chronic $\beta$-AR stimulation (Lehnart et al., 2005) and leads to spontaneous $\mathrm{Ca}^{2+}$ release from „leaky" RyR2 channels (Wehrens et al., 2003). Increased CaMKII activity increases RyR2 „leakiness" even more (Maier et al., 2007; Neef et al., 2010). In addition to PDE4D depletion in the RyR2 complex, whole cell changes in PDE3A, PDE4A and PDE4B expression and activity were observed in hypertrophied rat cardiomyocytes (Abi-Gerges et al., 2009). On the other hand, expression of PDE1, 2, 4 and 5 have been shown to be increased in early cardiac hypertrophy induced by chronic angiotensin II perfusion of rat hearts (Mokni et al., 2010).

Recently, it has been shown that $\beta_{1}$ AR stimulation leads to far reaching cAMP signals within cardiomyocytes, whereas $\beta_{2} A R$ stimulation remains locally defined at the T-tubular membranes (Nikolaev et al., 2006; Nikolaev et al., 2010). Besides, $\beta_{2}$ AR stimulation has no effect on PLN phosphorylation (Kuschel et al., 1999b). Combined scanning ion conductance microscopy (SICM) with FRET revealed that in failing rat cardiomyocytes, $\beta_{2}$-AR redistributed from the T-tubules to the call surface whereas $\beta_{1}$ AR localisation remained unchanged. This receptor redistribution was accompanied with diffuse $\beta_{2}$ cAMP signals throughout the cytosol, 
which might lead to increased PLN phosphorylation (Nikolaev et al., 2010).

Heart failure has been associated with a decrease in SR $\mathrm{Ca}^{2+}$ transport (Arai et al., 1993; Hasenfuss et al., 1994; Nagai et al., 1989) which might be due to decreased SERCA2a expression and activity and/or enhanced SERCA2a inhibition via PLN. Indeed, a dramatic drop in SERCA2a expression levels and activity has been observed in failing cardiomyocytes (Hasenfuss et al., 1994). SERCA2a gene transfer using a recombinant adeno-associated virus (AAV) has been shown to increase SERCA2a expression in rats, leading to an improvement of intracellular $\mathrm{Ca}^{2+}$ handling in vitro and in vivo in a rat model of heart failure (Houser et al., 2000; Jaski et al., 2009; Lyon et al., 2011). Furthermore, isolated cardiomyocytes from patients with end stage heart failure showed restored contraction and $\mathrm{Ca}^{2+}$ handling after SERCA2a adenoviral gene transfer and patients treated with AAV carrying SERCA2a showed improvement of heart parameters (Jessup et al., 2011). This AAV approach is now in phase 3 clinical trials.

PLN protein levels are not altered during cardiac disease (Meyer et al., 1995), whereas PLN phosphorylation is diminished, probably because of attenuated $\beta$-adrenergic signalling due to receptor desensitization, downregulation and uncoupling, which enhance SERCA2a inhibition via PLN (Barki-Harrington et al., 2004; Ferguson, 2001; Schwinger et al., 1999). Additionally, increased activation of PP-1 in human failing heart and experimental models of heart failure (Boknik et al., 2000; Neumann et al., 1997) has been shown to contribute to diminished PLN phosphorylation (Huang et al., 1999; Sande et al., 2002). This altered PP-1 activity might be due to differential regulation via $\mathrm{I}-1$. Indeed, studies in human and rat failing hearts uncovered a depressed phosphorylation of $\mathrm{I}-1$ at its Thr-35 site (El-Armouche et al., 2004; Gupta et al., 2005), possibly due to attenuated PKA activity. In addition to alterations in I-1 phosphorylation, decreased I-1 levels on the mRNA and protein level have been detected in human failing hearts (El-Armouche et al., 2004; El-Armouche et al., 2003) and canine and rat models for heart failure (El-Armouche et al., 2007; Gupta et al., 2003). These data suggest that $\mathrm{I}-1$ downregulation and inactivation might be a crucial event for increased PP-1 activity and hence, decreased PLN phosphorylation.

Interestingly, increased Hsp20 protein levels and increased Hsp20 phosphorylation in human diseased hearts have been detected (Qian et al., 2009). This might serve as a protective mechanism for counterbalancing increased PP-1 activity. Moreover, it has been shown that Hsp20 directly interacts with PDE4D. Disruption of this interaction leads to PKA dependent phosphorylation of Hsp20, thereby protecting against the hypertrophic response in neonatal rat cardiomyocytes after chronic $\beta$-AR stimulation (Sin et al., 2011).

All these mechanisms lead to decreased $\mathrm{SR} \mathrm{Ca}^{2+}$ content and less $\mathrm{Ca}^{2+}$ availability for each contraction round, thereby promoting the progression of cardiac disease to heart failure. 
Although alterations in global PDE activity have been reported in a rat model of cardiac hypertrophy and in human failing hearts (Abi-Gerges et al., 2009; Mehel et al., 2013), nothing is known about local changes in PDE composition around the SERCA2a microdomain. Besides, alterations in cAMP signalling and subsequent alterations in PKA activity seem to be crucial for the subsequent abnormalities in $\mathrm{Ca}^{2+}$ handling during cardiac disease. Therefore, cAMP dynamics in the SERCA2a microdomain and their differential regulation during cardiac disease such as hypertrophy are the object of intensive examination in this $\mathrm{PhD}$ thesis.

\subsection{Methods for cAMP detection in subcellular microdomains}

The widely accepted paradigm of compartmentalized cAMP signalling emphasizes the need for efficient detection methods that can resolve subcellular cAMP dynamics with high temporal and spatial resolution.

Biochemical assays such as radioimmunoassays or immunoblot analysis can be used for reliable direct or indirect measure of cAMP concentrations (Brooker et al., 1979; Harper and Brooker, 1975; Williams, 2004). However, these methods do not provide any insight into real time cAMP dynamics with spatial resolution under physiologically relevant conditions and consume a vast amount of cell material or tissue.

Jurevicius and colleagues used LTCC currents as an indirect read out for CAMP/PKA activity in frog cardiomyocytes. They could show that $\beta_{2} A R$ stimulation leads to locally confined CAMP signals that depend on PDE activity, while direct activation of ACs with forskolin led to a global increase in intracellular cAMP (Jurevicius and Fischmeister, 1996).

Another elegant tool for indirect visualization of cAMP increases within a cell are cyclic nucleotide gated channels (CNGCs) based sensors. Sarcolemmal CNGCs are activated upon cyclic nucleotide binding, thereby promoting a cation current and an increase in intracellular $\mathrm{Ca}^{2+}$ (Frings et al., 1995) which can be measured via patch clamp technique or using $\mathrm{Ca}^{2+}$ sensitive dyes, respectively (Abi-Gerges et al., 2009; Ghigo et al., 2012; Rochais et al., 2004). CNGCs based sensors contributed significantly to the clarification of some molecular mechanisms behind cAMP compartmentation at the plasma membrane, such as PDE3 and PDE4 dependent regulation of $\beta_{2} A R$ associated cAMP signals (Rochais et al., 2004) but their restriction to only one cellular compartment points out the necessity for more diverse localised cAMP biosensors.

One promising tool for direct visualization of different subcellular cAMP compartments in living cells are fluorescence resonance energy transfer (FRET) based cAMP biosensors that are described in more detail in the next section. 


\subsubsection{Fluorescence resonance energy transfer based cAMP biosensors}

Fluorescent resonance energy transfer (FRET) is a non-radiative energy transfer, whereby a donor fluorophore is excited by light of a specific wavelength and transfers its emission energy to an acceptor fluorophore (Förster, 1948). This leads to a reduction of the donor fluorescence and an increase in the acceptor emission intensity. A crucial requirement for this phenomenon to occur is that the emission spectrum of the donor fluorophore should overlap with the excitation spectrum of the acceptor fluorophore (Figure 5A). To allow proper energy transfer, the two fluorophores, usually variants of the green fluorescent protein (GFP) (Tsien, 1998), need to be in a favourable spatial orientation with a close proximity of less than $10 \mathrm{~nm}$ (Wu and Brand, 1994). According to the Jablonski-diagram (Figure 5B), photon absorption by the donor fluorophore lifts the donor electrons from the singlet state $\left(\mathrm{S}_{0}\right)$ to an excited state $\left(S_{1}\right)$. As the excited electrons "fall back" to their ground state, energy is emitted as fluorescence that can be partially absorbed by an acceptor fluorophore to lift the acceptor electrons from $S_{0}$ to the $S_{1}$ state. Again, fluorescence is emitted as the acceptor electrons return to the $S_{0}$ state.

A

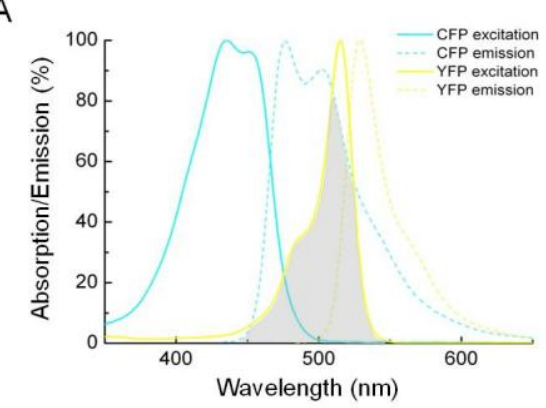

B

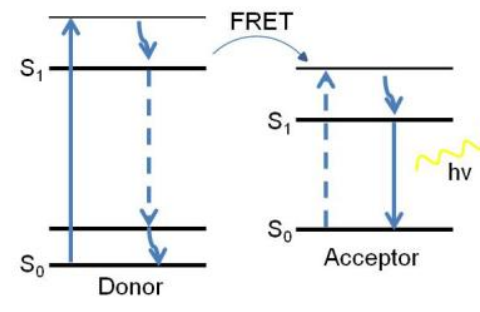

C

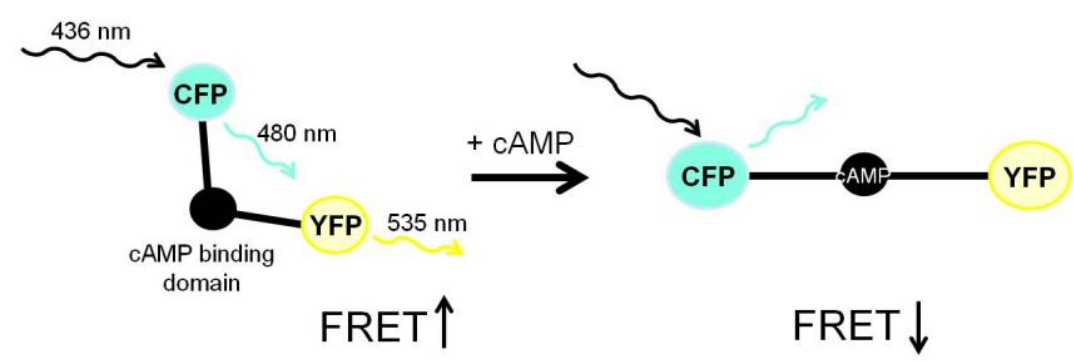

Figure 5. FRET mechanism using the CFP-YFP FRET pair. (A) The emission of the donor fluorophore (CFP) has to overlap with the excitation spectrum of the acceptor fluorophore (YFP) to allow fluorescence resonance energy transfer (FRET). The overlapping spectra are coloured grey. (B) Schematic representation of the Jablonski-diagram: after absorption of photon energy, the donor electrons are transitioned from a ground state $\left(S_{0}\right)$ to an excited state $\left(S_{1}\right)$. The donor fluorophore can partly transfer its energy from its lowest $S_{1}$ level to the electrons of an acceptor fluorophore with a lower energetic excitation spectrum (fluorophore distance must be $\leq 10 \mathrm{~nm}$ ). Acceptor electrons are lifted from their $S_{0}$ to the $S_{1}$ state and fluorescence (hv) is emitted when the acceptor electrons "fall back" into their $\mathrm{S}_{0}$ state. (C) Cartoon of FRET between CFP (donor) and YFP (acceptor). In the absence of CAMP, the two fluorophores are in close proximity and FRET occurs leading to quenched CFP emission and increased YFP emission. Upon cAMP binding to the sensor, the two fluorophores move apart, FRET is disrupted and YFP emission decreases while CFP emission increases. 
One prominent FRET pair that meets this requirement are CFP (cyan fluorescent protein) and YFP (yellow fluorescent protein), where CFP represents the donor and YFP the acceptor fluorophore that quenches CFP emission (Figure 5C). The degree of FRET can be measured via ratiometry by detecting the emission intensities of the donor and acceptor fluorophores and calculating a donor/acceptor or acceptor/donor ratio which describes the FRET signal. Several biosensors for cAMP have been generated based on this technique (Sprenger and Nikolaev, 2013).

PKA is one of the essential CAMP downstream targets which mediates phosphorylation of several effectors in different cellular compartments. The first cAMP FRET biosensor named FICRhR (abbreviation for "fluorescin-labelled PKA catalytic subunit and rhodamine-labelled regulatory subunit", "flicker") was produced by Roger Tsien and co-workers as a huge sensor complex consisting of chemically labelled R and C PKA subunits (Adams et al., 1991). To circumvent complex purification procedures and sensor microinjection, genetically encoded PKA based biosensors, using R and C PKA subunits fused to CFP and YFP, respectively, were first developed by Zaccolo and co-workers (Zaccolo et al., 2000; Zaccolo and Pozzan, 2002). This sensor allowed the first cAMP measurements in subcellular compartments at the Z-lines in neonatal rat ventricular myocytes (NRVMs), thereby uncovering the importance of PDE3 and PDE4 in cAMP compartmentation within NRVMs (Mongillo et al., 2004). Besides, measurements in adult rat cardiomyocytes were possible due to adenoviral transduction with this biosensor (Warrier et al., 2005). In another study, differentially localised PKA as well as Epac1 based FRET sensors expressed in HEK293A cells showed that PDEs might act as CAMP sinks creating different, independent subcellular pools of different cAMP concentrations (Terrin et al., 2006). To gain reasonable results with the tetrameric PKA based FRET sensors, equal expression of the sensor subunits is an inescapable requirement and the cooperative binding of cAMP to the different subunits results in relatively slow sensor kinetics. Besides, sensor subunits might associate with wildtype (WT) PKA subunits, thereby attenuating and/ or decelerating the actual FRET responses (Diller et al., 2001; Nikolaev et al., 2004). To avoid these problems, single chain cAMP biosensors were generated which contain just one single cAMP binding domain of the PKA-R subunit, such as PKA-camps (PKA-cAMP sensor) (Nikolaev et al., 2004). Another group of cAMP FRET biosensors are the A-kinase activity reporters (AKAR1-4) which contain a PKA phosphorylation site. Phosphorylation at this site leads to a change in the FRET signal and gives an indirect measure of PKA activity and cAMP changes in the cell (Allen and Zhang, 2006; Depry et al., 2011; Zhang et al., 2005; Zhang et al., 2001). The AKAR3 sensor has been fused to the transmembrane domain of PLN, leading to a localisation of this SR-AKAR3 to the SR membrane. Here, high PDE4 activity was detected which regulates PKA activity under basal conditions in NRVMs and adult rat ventricular myocytes transduced with SR-AKAR3 
adenovirus (Liu et al., 2011).

In addition to the PKA dependent FRET biosensors, further single chain CAMP FRET sensors have been developed based on the Epac protein. For example, the CFP-Epac-YFP sensors contain either a full-length or truncated Epac1 sequence and are located in the cytosol and to some extend at the nuclear envelope when expressed in mammalian cells (Ponsioen et al., 2004). The group around Jin Zhang also used full length Epac1 or truncated Epac2 and fused it to CFP and Citrine to generate ICUE (indicator of cAMP using Epac) FRET probes. The ICUE1 sensor construct localised to the cytosol and was also used to generate several targeted versions for subcellular specific cAMP measurements (DiPilato et al., 2004). The ICUE2 sensor represents an improved version of the ICUE1 construct showing larger FRET signals (Violin et al., 2008). Another prominent group of Epac based single chain cAMP biosensors are the Epac1-camps and Epac2-camps sensors that use a single CAMP binding domain of either human Epac1 or murine Epac2, respectively, fused to CFP and YFP (Nikolaev et al., 2004). Epac1-camps showed a slightly lower affinity for cAMP than Epac2-camps (2 and $1 \mu \mathrm{M}$, respectively) but larger FRET changes in Epac1-camps made it the preferred tool for subsequent studies. Epac1-camps is evenly distributed throughout the cytosol of mammalian cells and was used to detect cAMP diffusion in neurons and macrophages with a speed amounted to $40 \mu \mathrm{m} / \mathrm{s}$. This sensor was also used to generate a transgenic (TG) mouse model (CAG-Epac1-camps) with ubiquitous sensor expression to enable the detection of cAMP dynamics under highly physiological conditions (Calebiro et al., 2009). Several fusion proteins with Epac1-camps have been generated. For example, when fused to Hsp20, the new Epac1-camps biosensor uncovered the existence of a PDE4-Hsp20 complex in NRVMs (Sin et al., 2011). In another study, Epac1-camps was fused to PKA-RI (RI-epac) and PKA-RII (RII-epac) N-terminal dimerization and docking domains to directly monitor cAMP dynamics in the localisation sites of the different PKA isoforms within NRVMs. Here, $\beta$-adrenergic stimulation led to FRET changes exclusively in the RII-epac sensor associated with PLN and Tnl phosphorylation, whereas stimulation with PGE1 only stimulated RI-epac molecules (Di Benedetto et al., 2008). Epac1-camps was also combined with PDE3 and PDE4 to monitor cAMP dynamics in direct vicinity to these PDEs (Herget et al., 2008).

The group around Viacheslav Nikolaev developed another TG mouse model expressing the so called HCN2-camps sensor cardiac specific. This sensor contains a single cAMP binding domain from the murine hyperpolarization activated cyclic nucleotide gated potassium channel 2 (HCN2) fused to CFP and YFP. Using freshly isolated cardiomyocytes from this sensor mouse, far reaching $\beta_{1}$-AR-associated cAMP signals have been detected, whereas $\beta_{2}$-AR signals were found strictly confined at the cell membrane (Nikolaev et al., 2006). 
In the current PhD study, the cytosolic Epac1-camps cAMP biosensor was fused to the $\mathrm{N}$ terminus of full length PLN to allow the direct detection of CAMP dynamics within the SERCA2a compartment of adult ventricular mouse cardiomyocytes (Figure 6). Upon cAMP binding, the two fluorophores CFP and YFP move apart leading to a decrease of the FRET signal represented in this thesis as an increase of the donor/acceptor ratio (CFP/YFP).

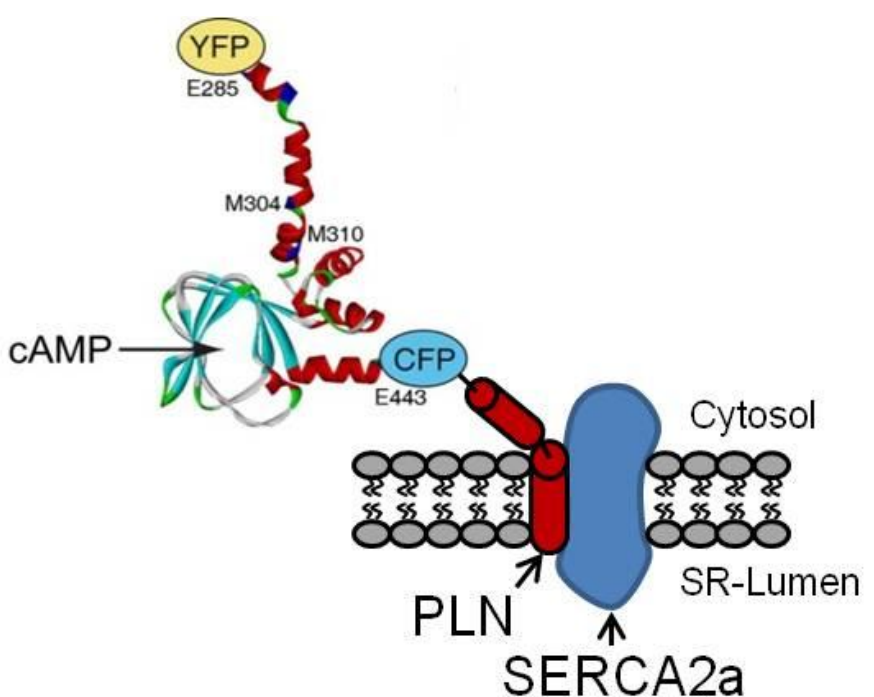

Figure 6. Cartoon of the Epac1-PLN biosensor and its estimated localisation to the SERCA2a microdomain within TG cardiomyocytes.

\subsection{Aims of this PhD thesis}

The aim of this $\mathrm{PhD}$ thesis was to generate a novel mouse model expressing the SERCA2a targeted cAMP FRET biosensor Epac1-PLN in a cardiac specific manner. Freshly isolated cardiomyocytes of this mouse line should be used for real time FRET measurements of local cAMP dynamics in healthy and diseased cardiomyocytes. As molecular alterations in the SERCA2a microdomain are associated with cardiac disease, the novel mouse line could be used as an in vivo model for cardiac hypertrophy to analyse altered SERCA2a-associated cAMP signals in the diseased cardiomyocytes. To understand the molecular mechanisms behind CAMP compartmentation in the SERCA2a microdomain, FRET results gained in Epac1-PLN cardiomyocytes should be compared with those gained in cardiomyocytes expressing the cytosolic Epac1-camps cAMP FRET sensor. These measurements would help to shed light on the role of local cAMP changes for disease progression.

In a side project, $\mathrm{Ca}^{2+}$ influence on FRET cAMP measurements using the cytosolic FRET sensor Epac1-camps should be investigated. It is known, that $\mathrm{Ca}^{2+}$ influences several important cAMP regulating enzymes within the cell. Therefore, it was important to analyse whether rapid $\mathrm{Ca}^{2+}$ changes during diastole and systole translate into cAMP changes recorded using the FRET measurements. 


\section{Materials and Methods}

\subsection{Materials}

\subsubsection{Cells}

HEK293A

Invitrogen, \#R705-07

\subsubsection{Plasmids}

pcDNA3.0

Invitrogen (Life Technologies)

$\alpha-\mathrm{MHC}$

Nikolaev et al., 2006

Epac1-PLN (pcDNA3.0)

AG Lohse, Würzburg

truncated Epac1-PLN (pcDNA3.0)

AG Lohse, Würzburg

Epac1-camps R279E (pcDNA3.0)

AG Lohse, Würzburg

Epac1-PLN R279E (pcDNA3.0)

this $\mathrm{PhD}$ thesis

Epac1-PLN dark YFP (pcDNA3.0)

this $\mathrm{PhD}$ thesis

Epac1-PLN dark CFP (pcDNA3.0)

this $\mathrm{PhD}$ thesis

Epac1-PLN ( $\alpha-\mathrm{MHC})$

this $\mathrm{PhD}$ thesis

Gateway® pDONR ${ }^{\text {TM }} 221$

Invitrogen

Gateway ${ }^{\circledR}$ pAd/CMV/V5-DEST ${ }^{\mathrm{TM}}$

Invitrogen

\subsubsection{Bacteria strains}

One Shot ${ }^{\circledR}$ TOP10 chemically competent $E$. coli Invitrogen One Shot® OmniMax2T1 Invitrogen

\subsubsection{Animals}

FVB/NRj mice were obtained from Janvier Labs (Saint Berthevin, France) and used for pronuclear injection with the Epac1-PLN construct by the Max Planck Institute of Experimental Medicine. All animal experiments were performed in accordance with institutional and governmental guidelines. 


\subsubsection{Oligonucleotides}

The rabbit PLN sequence was kindly provided by Dr. med. Joachim Schmitt, Würzburg.

Rabbit PLN sequence

5"- ATG GAG AAA GTT CAA TAC CTC ACT CGC TCT GCT ATA AGA AGG GCC TCA ACC ATT GAA ATG CCT CAA CAA GCA CGT CAA AAC CTC CAG AAC CTA TTT ATC AAT TTC TGT CTC ATC TTG ATA TGT CTC CTG CTG ATC TGC ATC ATC GTC ATG CTT CTC TGA-3"

All other oligonucleotides were purchased from MWG Biotech $\mathrm{GmbH}$, Ebersberg

CFPXbalFor

CFPBamHIRev

GAPDHFor

GAPDHRev

MHCseqFor

pcDNA3attBFor

pcDNA3attBRev

PLBBamP4linHaseFor

PLBXhostHaseRev

PLNFor

PLNRev

TruncNhelFor

YFPRev
5"-AAATCTAGAGTGAGCAAGGGCGAGG-3"

5"-AAAGGATCCCTTGTACAGCTCGTCCATG-3"

5"-GTTGTCTCCTGCGACTT-3"

5"- GGTCCAGGGTTTCTTACT-3"

5"-TGACAGACAGATCCCTCCTAT-3"

5"-GGGGACAAGTTTGTACAAAAAAGCAGGCTGACTC ACTATAGGGAGACCC-3"

5"-GGGGACCACTTTGTACAAGAAAGCTGGGTAGCGA GCTCTAGCATTTAGG-3"

5"-AAGGATCCATGCCCTTGGTGGATTTCTTCTGCGA GAAAGTTCAATAC-3"

5"-AAACTCGAGTCAGAGAAGCATGACGAT-3"

5"-CAATACCTCACTCGCTC-3"

5"-ATGATGCAGATCAGCAG-3"

5"- AAAGCTAGCGAAATGCCTCAGCAAGCAC-3, 5"-CATGGCGGACTTGAAGAAGT-3"

\subsubsection{Chemicals}

AG 1-X8 Resin

Albumin Fraction $\mathrm{V}$

Ammonium persulfate

Ampilcillin

Ampuwa ${ }^{\circledR}$ water

BAY 60-7550

BES buffer grade

$\beta$-Mercaptoethanol
Biorad, \# 140-1441

Applichem, \# A1391.0100

Sigma, \# A3678

Roth, \# K029.1

Fresenius Kabi Deutschland $\mathrm{GmbH}$

Santa Cruz, \# sc-205219

Applichem, A1062

Sigma, \# M3148 
Bromphenol Blue sodium salt

8-Br-2"-O-Me-cAMP-AM

2,3- Butanedione monoxime

Caffeine

Calcium chloride dihydrat

Calcium ionophore A23187

cAMP

CAMP $\left[5,8-{ }^{3} \mathrm{H}\right]$

Cesium chloride

CGP-20712A methanesulfonate salt

Cilostamide

Crotalus atrox Snake venom

Dimethyl Sulphoxide HYBRI-MAX ${ }^{\circledR}$

DirectPCR-Tail

$\mathrm{D}(+)$ Sucrose

dNTPs

EDTA

EGTA

Ethanol Rotipuran $>99,8 \%$

Ethidium bromide - Solution 1\%

Fluo3-AM

Forskolin

Fura2-AM

Glucose

Glycerol

Glycine

$\mathrm{H}-89$ dihydrochloride hydrate

Hematoxylin

HEPES

Hydrochloride acid 37\%

ICl-118.551 hydrochloride

Iron(III) chloride hexahydrate

3-Isobutyl-1-methylxanthin

Isoproterenol hydrochloride

Laminin

LB- Agar powder Miller

LB- Medium powder Miller

Loading buffer DNA IV (for Agarose gels)

Magnesium chloride hexahydrate
Applichem, \# A1120

Biolog, \# B028

Sigma, \# B0753

Sigma, \# C0750

Merck, \# 17257

Sigma \# C7522

Sigma, \# A9501

Hartmann analytic $\mathrm{GmbH}$, \# 1790

Sigma, \# C3032

Sigma, \# C231

Sigma, \# C7971

Sigma, \# V7000

Sigma, \# D2650

Peqlab, \# 31-102-T

Roth, \# 4621.2

Promega, \# U1240

Roth, \# 8040.3

Sigma, \# E4378

Roth, \# 9065.1

Applichem, \# A1152

Invitrogen, \# F-1242

Sigma, \# F6886

Invitrogen, \# F-1201

Sigma, \# G7021

Sigma, \# G8773

Roth, \# 3908.3

Sigma, \# B1427

Fluka \# 51260

Sigma, \# H4034

Sigma, \# 84422

Sigma, \# I127

Roth, \# P742.1

Applichem, \# A0695

Sigma, \# 16504

Sigma, \# L2020

Applichem, \# A0927

Applichem, \# A0954

Applichem, \# A3481

Applichem, \# A1036 
Magnesium sulfate heptahydrate

MDL-12,330A hydrochloride

Methanol

8-methoxymethyl-3-isobutyl-1-methylxanthine

Milkpowder

$N, N, N^{\prime}, N^{\prime}$-Tetramethylethylenediamine

peqGOLD Universal Agarose

PhosStop

Ponceau S

Potassium bicarbonate

Potassium chloride

Potassium dihydrogen phosphate

RNAse free water

Phenol red sodium salt

Protease Inhibitor Cocktail

Protein Marker V

Quick-Load $^{\circledR}$ 100bp DNA ladder

Quick load $^{\circledR} 1$ kb DNA ladder

Rolipram

Roticlear $^{\circledR}$

Roti-Histofix ${ }^{\circledR} 4 \%$

Rotiphorese ${ }^{\circledR}$ Gel 30

Sodium azide

Sodium bicarbonate

Sodium chloride

Sodium dodecyl sulfate solution $20 \%$

Sodium hydroxide

Sodium phosphate dibasic

Sodium phosphate dibasic dihydrate

Sucrose

TAE-buffer (50x)

Target Retrieval Solution, Citrate pH6 (10x)

Taurine

Thapsigargin

TRIS

Triton- $X^{\circledR} 100$

Tween-20 ${ }^{\circledR}$

Vectashield ${ }^{\circledR}$ Mounting Medium

Lectin from Triticum vulgaris (wheat)
Sigma, \# M2773

Sigma, \# M182

Roth, \# HN41.2

Sigma, \# M2547

Roth, \# T145.1

Sigma, \# T9281

Peqlab, \# 35-1020

Roche, \# 04906837001

Sigma, \# P3504

Sigma, \# P7682

Sigma, \# P5405

Merck, \# 4873

Ambion, \# AM9937

Sigma, \# P5530

Roche, \# 11872580001

Peqlab, \#27-2211

Biolabs, \# NO467S

Biolabs, \# NO468S

Sigma, \# R6520

Roth, \# A538.5

Roth, \# P087.5

Roth, \# 3029.1

Sigma, \# S2002

Sigma, \# S5761

Sigma, \# S5886

Fluka, \# 05030

Roth, \# 6771.3

Sigma, \# 255793

Sigma, \# 71643

Sigma, \# S0389

Applichem, \# A1691

Dako, \# S2369

Sigma, \# T8691

Sigma, \# T9033

Roth, \# 4855.3

Applichem, \# A1287.0025

Sigma, \# P1379

Vector Laboratories, \# H-1000

Sigma, \# L5266 


\subsubsection{Cell culture}

Antibiotic-Antimycotic, 100x

DMEM, $4.5 \%$ glucose

FCS

Glutamine

Iscove Basal Medium

Lipofectamine ${ }^{\circledR} 2000$ Reagent

OPTI-MEM ${ }^{\circledR}$

PBS Phosphate Buffered Saline (Dulbecco)

Penicillin/Streptomycin

Plaque GP Agarose

Trypsin/ EDTA solution
Gibco, \# 15240062

Biochrom, \# F0445

Biochrom, \# S0615

Biochrom, \# K0283

Biochrom, \# FG 0465

Invitrogen, \# 11668

Gibco, \# 11058

Biochrom, \# L1825

Biochrom, \# A2213

Biozym, \# 850110

Biochrom, \# L2143

\subsubsection{Enzymes and Kits}

EcoRI

Gateway® BP Clonase II Enzyme Mix

Gateway ${ }^{\circledR}$ LR Clonase ${ }^{\text {TM }}$ II Enzyme Mix

GoTaq DNA Polymerase, $500 U$

iScript cDNA Synthesis Kit

Kpnl

Liberase DH

Notl

Pacl

Pfu DNA Polymerase

Pierce BCA Protein Assay Kit

Plasmid Midi Kit

Plasmid Mini Kit

Proteinase $\mathrm{K}$

QiaShredder

QIAquick Gel Extraction Kit

QIAquick PCR purification Kit

Qproteome Cell compartment Kit

RNeasy Fibrous Tissue Mini Kit

Sodiumacetate solution $3 \mathrm{M}$
New England Biolabs, \# R0101

Invitrogen, \# 11789-020

Invitrogen, \# 11791-020

Promega, \# M3175

Biorad, \# 170-8890

New England Biolabs, \# R3142

Roche, \# 05401054001

New England Biolabs, \# R3189

New England Biolabs, \# R0547

Promega, \# M774B

Thermo Scientific, \# 23227

Qiagen, \# 12945

Qiagen, \# 12125

Applichem, \# A3830-0500

Qiagen, \# 79654

Qiagen, \# 28704

Qiagen, \# 28104

Qiagen, \# 37502

Qiagen, \# 74704

Applichem. \# 3947 
Super Signal West Pico Chemol. Substrate SYBR $^{\circledR}$ Green Super Mix for $\mathrm{iQ}^{\mathrm{TM}}$

T4 DNA Ligase

Trypsin 2,5\%

Xhol
Thermo Scientific, \# 34080

Quanta Biosciences, \# 95053

NEB, \# M0202S

Gibco \#15090

New England Biolabs, \# R0146

\subsubsection{Antibodies}

Table 1 Primary antibodies for Immunoblot (WB) and Immunhistology (IH). Buffer for antibody dilution was prepared in TBS-T (WB) or in PBS $(\mathrm{IH})$ including milk powder or BSA

\begin{tabular}{|c|c|c|}
\hline Antibody & Dilution & \\
\hline anti- Calsequestrin & $\begin{array}{l}\text { WB } 1: 10.000 \\
\text { in } 3 \% \text { BSA }\end{array}$ & Thermo Scientific \# PA1-913 \\
\hline anti- Cav1.2a & $\begin{array}{l}\text { WB 1:160 } \\
\text { in 5\% milk }\end{array}$ & Alomone Labs \# ACC-013 \\
\hline anti- GAPDH & $\begin{array}{l}\text { WB 1:36.000 } \\
\text { in } 5 \% \text { milk }\end{array}$ & HyTest Ltd, \# 5G4 \\
\hline anti- GFP & $\begin{array}{l}\text { WB 1:500 } \\
\text { in } 5 \% \text { milk } \\
\text { IH } 1: 500 \\
\text { in } 1 \% \text { BSA }\end{array}$ & Santa Cruz, \# sc-9996 \\
\hline anti- NCX & $\begin{array}{l}\text { WB 1:5000 } \\
\text { in 5\% milk }\end{array}$ & Swant, \# R3F1 \\
\hline anti- PDE2A & $\begin{array}{l}\text { WB 1:750 } \\
\text { in } 3 \% \text { BSA }\end{array}$ & Fabgennix, \# 101AP \\
\hline anti- PDE4D & $\begin{array}{l}\text { WB 1:200 } \\
\text { in 5\% milk }\end{array}$ & Santa Cruz, \# sc-25814 \\
\hline anti- PDE4D8 & $\begin{array}{l}\text { WB 1:2000 } \\
\text { in 5\% milk }\end{array}$ & Gift by M. Conti, San Francisco \\
\hline anti- PKA-RI & $\begin{array}{l}\text { WB 1:500 } \\
\text { in 5\% milk }\end{array}$ & BD Transduction, \# 610165 \\
\hline Anti- PKA-RII & $\begin{array}{l}\text { WB 1:500 } \\
\text { in 5\% milk }\end{array}$ & BD Transduction, \# 612242 \\
\hline anti- PLN Phospho Serine-16 & $\begin{array}{l}\text { WB 1:5000 } \\
\text { in 3\% BSA }\end{array}$ & Badrilla, \# A010-12 \\
\hline anti- PLN Phospho Threonine-17 & $\begin{array}{l}\text { WB 1:5000 } \\
\text { in 5\% milk }\end{array}$ & Badrilla \# A010-13 \\
\hline anti- PLNA1 & $\begin{array}{l}\text { WB 1:5000 } \\
\text { in 5\% milk }\end{array}$ & Badrilla, \# A010-14 \\
\hline anti- PMCA Clone 5F10 & $\begin{array}{l}\text { WB 1:500 } \\
\text { in 5\% milk }\end{array}$ & Sigma \# P6363 \\
\hline anti- SERCA2a anti-Serum & $\begin{array}{l}\text { WB 1:5000 } \\
\text { in } 5 \% \text { milk } \\
\text { IH } 1: 100 \\
\text { in } 1 \% \text { BSA }\end{array}$ & Badrilla, \# A010-20 \\
\hline
\end{tabular}


Table 2 Secondary antibodies for Immunoblot (WB) and Immunhistology (IH). Buffer for antibody dilution was prepared as for the associated primary antibody

\begin{tabular}{l|l|l}
\hline Antibody & Dilution & \\
\hline & & \\
Alexa Fluor $~$ & & \\
Alexa Fluor $₫ 514$ Goat Anti-Rabbit IgG & IH $1: 300$ & Invitrogen, \# A21070 \\
Immun-Star Goat Anti-Mouse (GAM)-HRP & IH 1:500 & Invitrogen, \# A31555 \\
Immun-Star Goat Anti-Rabbit (GAR)-HRP & WB 1:5000 & Biorad, \# 170-5047 \\
& & Biorad, \# 170-5046 \\
\hline
\end{tabular}

\subsubsection{Microscope devices and software}

Arduino I/O board

Attofluor® cell chamber

AxioObserver A1 epifluorescence microscope

AxioCam ICc1

Axiovert 200 microscope

Axio Vision software

Binocular macroscope

CFP/YFP filter set

CoolLED $440 \mathrm{~nm}$

CooISNAP-HQ CCD-camera

DualView filter slider

DV2 DualView (505dcxr filter)

ImageJ Software

Inverted fluorescent microscope

710 NLO microscope

Microsoft Office Picture Manager

Oil immersion $63 x$ objective

ORCA-03G camera

Polychrome $\mathrm{V}$ light source

Stemi 2000-C microscope binocular

ZEN 2010 Software
Sparkfun Electronics

Invitrogen

Carl Zeiss Microlmaging

Carl Zeiss Microlmaging

Carl Zeiss Microlmaging

Carl Zeiss Microlmaging

Olympus

Chroma Technology

CoolLED

Visitron Systems

Photometrics

Photometrics

National Institutes of Health

Nikon

Carl Zeiss Microlmaging

Microsoft Corporation

Carl Zeiss Microlmaging

Hamamatsu Photonics

TILLPhotonics

Carl Zeiss Microlmaging

Carl Zeiss Microlmaging 


\subsubsection{Calcium measurement devices and software}

Fluorescence System Interface

Fluo-3 Filter

Fura-2 Filter

Hyper Switch Light Source

IonWizard- Core and Analysis

MyoCam-S

MyoPacer Cell Stimulator

Sarcomere Length Acquisition Module
IonOptix

Chroma Technology

Chroma Technology

IonOptix

IonOptix

IonOptix

IonOptix

IonOptix

\subsubsection{General devices and software}

Alphalmager ${ }^{\circledR}$ software

Biotek Reader (for BCA assay)

Centrifuges

MicroBeta $^{2}$

MicroBeta ${ }^{2}$ Windows Workstation

MS-400 MicroScan Transducer

Multilmage Light Cabinet

NanoDrop 2000

Powerpac HC

Thermocycler

ThermoMix compact

iCycler

Microtom Leica RM 2165

Mini-PROTEAN ${ }^{\circledR}$ Electrophoresis System

Mupid-One Gel Electrophoresis Unit

Origin Pro 8.5 Software

$\mathrm{pH}$ meter

Tracheal tube

Ultracentrifuge L-70

Ultra-Turrax MicraD-1

UV Table IL-350-M

Vevo 2100
ProteinSimple

BIOTEK Instruments

Thermo Scientific

Perkin Elmer, Inc.

Perkin Elmer, Inc.

Linear Array Technology

Alpha Innotech Corporation

Thermo Scientific

Biorad

Sensoquest

Eppendorf

Biorad

Leica

Biorad

ADVANCE Co., Ltd.

OriginLab Corporation

Inolab

Hugo Sachs Electronic

Beckman

Art-Labortechnik

Bachofer

VisualSonics (Toronto, Canada) 
Ventilator Minivent

X-Ray Film processor SRX 101A
Hugo Sachs Electronic

Konica

\subsubsection{Other materials}

Dialysis Tubing for virus dialysis

Elca ${ }^{\circledR}$ med

Eppendorf tubes

Ethilon suture 9-0

Falcon tubes

Fiber pads for Western blot

Filter Unit 0.2 RC Spartan $130.2 \mu \mathrm{m}$ (DNA filtration)

Forene $^{\circledR}$

21-gauge needle

26-gauge needle

Gauze

Glass Cover Slips 24 mm

Microscope Slides Thermo Scientific,

Medical X-Ray Film

Prolene suture 6-0

Protran Nitrocellulose Transfer Membrane

Quickseal Centrifuge Tubes (virus centrifugation)

Scintilation Liquid Lumasafe Plus

Serological pipettes

Slide-A-Lyzer Dialysis Cassettes, 10K MWCO

Spacer Plates for Western blot

Steriflips

Short Plates for Western blot

Temgesic $®$

U-40 Insulin 30Gx1/2

U-40 Insulin Omnifix Solo

Water bath

6 Well Plates

96 Well Plates

96 Well Plate for MicroBeta
Medical International Ltd.

Asid Bonz GmbH

Eppendorf

Ethicon

BD Falcon

Bio Rad, \#1703933

Whatman, \# 10463040

Abbott

BD Microlane

BD Microlane

Th Geyer, \# 9.068291

Thermo Scientific, \# 004710781

\# J1800AMNZ

Fujifilm, \# 4014403

Ethicon

Whatman, \# 4018650

Beckmann, \# 342413

Lumac LSC, \# 3097

Sarstedt

Thermo Scientific, \# $663830.5 \mathrm{ml}$

Bio Rad, \#1653311

Millipore, \# SCGP00525

Bio Rad, \#1653308

Essex Pharma GmbH

Braun, \# 40012525

Braun, \# 9161309v

Julabo

Starlab, \# CC7682-7506

Nunc, \# 167008

Perkin Elmer, Inc. \#1450-401 


\subsubsection{Buffers}

All buffers were prepared in aqua dest.

Table 3 Buffer for plasmid dialysis

\begin{tabular}{l|ll}
\hline Buffer & Composition \\
\hline \multirow{3}{*}{ TE Buffer } & Tris, 1M, pH 7.4 & $5 \mathrm{ml}$ \\
& EDTA, 0.5 M, pH 8 & $0.2 \mathrm{ml}$ \\
& Ampuwa & $1000 \mathrm{ml}$ \\
& sterile under hood! & \\
\hline
\end{tabular}

Table 4 Buffer and media for E.coli transformation

\begin{tabular}{|c|c|c|}
\hline Buffer & Composition & \\
\hline $5 \times$ KCM buffer & $\begin{array}{l}\mathrm{CaCl}_{2} \\
\mathrm{MgCl}_{2} \\
\mathrm{KCL}\end{array}$ & $\begin{array}{l}150 \mathrm{mM} \\
250 \mathrm{mM} \\
500 \mathrm{mM}\end{array}$ \\
\hline LB medium & $\begin{array}{l}\text { LB medium powder } \\
\text { sterilized by autoclaving } \\
\text { Ampicillin }\end{array}$ & $\begin{array}{l}25 \mathrm{~g} / \mathrm{l} \\
100 \mu \mathrm{g} / \mathrm{ml}\end{array}$ \\
\hline LB plates & $\begin{array}{l}\text { LB agar powder } \\
\text { sterilized by autoclaving } \\
\text { Ampicillin }\end{array}$ & $\begin{array}{l}40 \mathrm{~g} / \mathrm{l} \\
100 \mu \mathrm{g} / \mathrm{ml}\end{array}$ \\
\hline
\end{tabular}

Table 5 Solutions for HEK293A cell transfection

\begin{tabular}{l|ll}
\hline Buffer & Composition & \\
\hline & & \\
2x BBS & $\mathrm{Na}_{2} \mathrm{HPO}_{4}$ & $1.5 \mathrm{mM}$ \\
sterile filtrate & $\mathrm{BES}$ & $50 \mathrm{mM}$ \\
& $\mathrm{NaCl}$ & $280 \mathrm{mM}$ \\
& $p H$ & 6.95 with $\mathrm{NaOH}$ \\
\hline
\end{tabular}




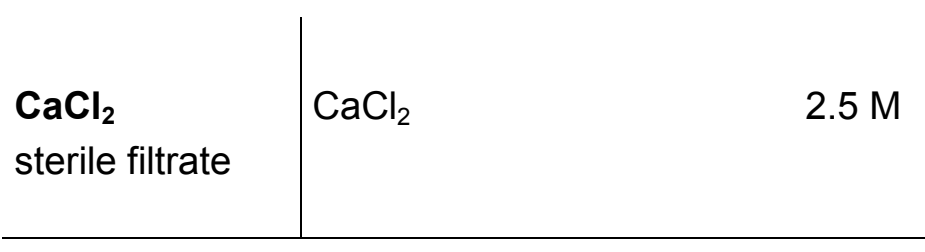

Table 6 Solutions for adenovirus purification

\begin{tabular}{l|ll}
\hline Buffer & Composition \\
\hline \multirow{3}{*}{ 10x Sucrose buffer } & Tris & $24.22 \mathrm{~g}$ \\
sterile filtrate & $\mathrm{MgCl}_{2} \times 6 \mathrm{H}_{2} \mathrm{O}$ & $8.13 \mathrm{~g}$ \\
& $\mathrm{D}(+)$ Sucrose & $800 \mathrm{~g}$ \\
& $\mathrm{pH}$ & 8 \\
& Aqua dest. & ad $2000 \mathrm{ml}$ \\
\hline
\end{tabular}

Table 7 Solutions for cardiomyocyte isolation

\begin{tabular}{|c|c|c|}
\hline Buffer & Composition & \\
\hline $\begin{array}{l}\text { Stock Perfusion Buffer } \\
10 x \\
\text { sterile filtrate }\end{array}$ & $\begin{array}{l}\mathrm{NaCl} \\
\mathrm{KCl} \\
\mathrm{KH}_{2} \mathrm{PO}_{4} \\
\mathrm{Na}_{2} \mathrm{HPO}_{4} \times 2 \mathrm{H}_{2} \mathrm{O} \\
\mathrm{MgSO}_{4} \times 7 \mathrm{H}_{2} \mathrm{O} \\
\mathrm{Phenol} \mathrm{red} \\
\mathrm{NaHCO}_{3} \\
\mathrm{KHCO}_{3} \\
\mathrm{HEPES} \\
\text { Taurine }\end{array}$ & $\begin{array}{l}1.13 \mathrm{M} \\
47 \mathrm{mM} \\
6 \mathrm{mM} \\
6 \mathrm{mM} \\
12 \mathrm{mM} \\
0.32 \mathrm{mM} \\
120 \mathrm{mM} \\
100 \mathrm{mM} \\
100 \mathrm{mM} \\
300 \mathrm{mM}\end{array}$ \\
\hline $\begin{array}{l}\text { Perfusion Buffer } 1 \mathbf{x} \\
\text { sterile filtrate }\end{array}$ & $\begin{array}{l}\text { Stock Perfusion Buffer } 10 x \\
\text { BDM solution } \\
\text { Glucose } \\
\text { Aqua dest. }\end{array}$ & $\begin{array}{l}10 \mathrm{ml} \\
2 \mathrm{ml} \\
100 \mathrm{mg} \\
\text { ad } 100 \mathrm{ml}\end{array}$ \\
\hline BDM Solution & BDM & $500 \mathrm{mM}$ \\
\hline $\begin{array}{l}\text { BSA Solution } \\
\text { sterile filtrate }\end{array}$ & BSA & $10 \%(w / v)$ \\
\hline
\end{tabular}




\begin{tabular}{|c|c|c|}
\hline $\begin{array}{l}\mathrm{CaCl}_{2} \text { Solution } 100 \mathrm{mM} \\
\text { sterile filtrate }\end{array}$ & $\mathrm{CaCl}_{2}$ & $100 \mathrm{mM}$ \\
\hline $\begin{array}{l}\mathrm{CaCl}_{2} \text { Solution } 10 \mathrm{mM} \\
\text { sterile filtrate }\end{array}$ & $\mathrm{CaCl}_{2}$ & $10 \mathrm{mM}$ \\
\hline $\begin{array}{l}\text { Liberase Solution } \\
\text { sterile conditions }\end{array}$ & $\begin{array}{l}\text { Liberase DH } \\
\text { Aqua dest. }\end{array}$ & $\begin{array}{l}50 \mathrm{mg} \\
12 \mathrm{ml}\end{array}$ \\
\hline Digestion Buffer & $\begin{array}{l}\text { Perfusion Buffer } 1 \mathrm{x} \\
\mathrm{CaCl}_{2} \text { solution } 100 \mathrm{mM} \\
\text { Trypsin } 2.5 \% \\
\text { Liberase Solution }\end{array}$ & $\begin{array}{l}2.6 \mathrm{ml} \\
3.75 \mu \mathrm{l} \\
200 \mu \mathrm{l} \\
300 \mu \mathrm{l}\end{array}$ \\
\hline Stopping Buffer 1 & $\begin{array}{l}\text { Perfusion Buffer 1x } \\
\text { BSA Solution } \\
\mathrm{CaCl}_{2} \text { Solution } 100 \mathrm{mM}\end{array}$ & $\begin{array}{l}2.25 \mathrm{ml} \\
250 \mu \mathrm{l} \\
1.25 \mu \mathrm{l}\end{array}$ \\
\hline Stopping Buffer 2 & $\begin{array}{l}\text { Perfusion Buffer 1x } \\
\text { BSA Solution } \\
\mathrm{CaCl}_{2} \text { Solution } 100 \mathrm{mM}\end{array}$ & $\begin{array}{l}9.5 \mathrm{ml} \\
500 \mu \mathrm{l} \\
3.75 \mu \mathrm{l}\end{array}$ \\
\hline
\end{tabular}

Table 8 Tyrode solutions

\begin{tabular}{l|ll}
\hline Buffer & \multicolumn{2}{|l}{ Composition } \\
\hline \multirow{3}{*}{ FRET buffer } & $\mathrm{NaCl}$ & $144 \mathrm{mM}$ \\
& $\mathrm{KCl}$ & $5.4 \mathrm{mM}$ \\
& $\mathrm{MgCl}_{2}$ & $1 \mathrm{mM}$ \\
$\mathrm{CaCl}_{2}$ & $1 \mathrm{mM}$ \\
& $\mathrm{HEPES}$ & $10 \mathrm{mM}$ \\
& $\mathrm{pH}$ & 7.3 \\
\hline \multirow{3}{*}{ Tyrode } & & \\
Ca ${ }^{2+}$ measurements & $\mathrm{NaCl}$ & $149 \mathrm{mM}$ \\
& $\mathrm{KCl}$ & $1 \mathrm{mM}$ \\
& $\mathrm{MgCl}$ & $1 \mathrm{mM}$ \\
& $\mathrm{HEPES}$ & $5 \mathrm{mM}$
\end{tabular}




$\begin{array}{ll}\text { Glucose } & 10 \mathrm{mM} \\ \mathrm{CaCl}_{2} & 1 \mathrm{mM} \\ \mathrm{pH} & 7.54\end{array}$

Table 9 Immunoblot solutions

\begin{tabular}{|c|c|c|}
\hline Buffer & Composition & \\
\hline Homogenization Buffer & $\begin{array}{l}\text { HEPES } \\
\text { Succrose } \\
\mathrm{NaCl} \\
\text { EGTA } \\
\mathrm{CaCl}_{2} \\
\text { Triton } \times 100_{\mathrm{pH}}\end{array}$ & $\begin{array}{l}10 \mathrm{mM} \\
300 \mathrm{mM} \\
150 \mathrm{mM} \\
1 \mathrm{mM} \\
2 \mathrm{mM} \\
10 \% \\
7.4 \\
10 \mathrm{ml}+1 \text { tablet PhosStop } \\
+1 \text { tablet Protease Inhibitor } \\
\text { Cocktail }\end{array}$ \\
\hline SDS Stop $3 x$ & $\begin{array}{l}\text { Tris } \\
\text { SDS } \\
\text { Glycerol } \\
\text { Bromphenol Blue } \\
\beta \text {-Mercapthoethanol } \\
\mathrm{pH}\end{array}$ & $\begin{array}{l}200 \mathrm{mM} \\
6 \%(\mathrm{v} / \mathrm{v}) \\
15 \%(\mathrm{v} / \mathrm{v}) \\
10 \%(\mathrm{v} / \mathrm{v}) \\
6.7\end{array}$ \\
\hline 4x Tris/SDS pH 6.8 & $\begin{array}{l}\text { Tris } \\
\text { SDS } \\
\mathrm{pH}\end{array}$ & $\begin{array}{l}500 \mathrm{mM} \\
0.4 \%(\mathrm{v} / \mathrm{v}) \\
6.8\end{array}$ \\
\hline 4x Tris/SDS pH 8.8 & $\begin{array}{l}\text { Tris } \\
\text { SDS } \\
\mathrm{pH}\end{array}$ & $\begin{array}{l}1.5 \mathrm{M} \\
0.4 \%(\mathrm{v} / \mathrm{v}) \\
8.8\end{array}$ \\
\hline APS solution & APS & $10 \%(w / v)$ \\
\hline 10x SDS Running Buffer & $\begin{array}{l}\text { Tris } \\
\text { Glycine } \\
\text { SDS }\end{array}$ & $\begin{array}{l}250 \mathrm{mM} \\
1.9 \mathrm{M} \\
1 \%(\mathrm{v} / \mathrm{v})\end{array}$ \\
\hline
\end{tabular}




\begin{tabular}{|c|c|c|}
\hline & $\mathrm{pH}$ & 8.3 \\
\hline 10x Transfer Buffer & $\begin{array}{l}\text { Tris } \\
\text { Glycine }\end{array}$ & $\begin{array}{l}325 \mathrm{mM} \\
1.9 \mathrm{M}\end{array}$ \\
\hline 1x Transfer Buffer & $\begin{array}{l}\text { 10x Transferbuffer } \\
\text { Methanol }\end{array}$ & $\begin{array}{l}10 \%(v / v) \\
20 \%(v / v)\end{array}$ \\
\hline Ponceau S Solution & $\begin{array}{l}\text { Ponceau S } \\
\text { in } 10 \% \text { acetic acid }\end{array}$ & $0.5 \%(w / v)$ \\
\hline 10x TBS Buffer & $\begin{array}{l}\text { Tris } \\
\mathrm{NaCl} \\
\mathrm{pH}\end{array}$ & $\begin{array}{l}100 \mathrm{mM} \\
1.5 \mathrm{M} \\
7.5(\mathrm{HCl})\end{array}$ \\
\hline 1X TBS-Tween Buffer & $\begin{array}{l}\text { 10x TBS Buffer } \\
\text { Tween } 20\end{array}$ & $\begin{array}{l}10 \%(v / v) \\
0.1 \%(v / v)\end{array}$ \\
\hline $\begin{array}{l}\text { Stacking Gel } \\
(3.8 \mathrm{ml} ; 2 \text { Gels) }\end{array}$ & $\begin{array}{l}\text { Acrylamide } \\
4 x \text { Tris/SDS pH } 6.8 \\
\text { Aqua dest. } \\
10 \% \text { APS } \\
\text { TEMED }\end{array}$ & $\begin{array}{l}500 \mu \mathrm{l} \\
940 \mu \mathrm{l} \\
2.31 \mathrm{ml} \\
18.8 \mu \mathrm{l} \\
7.5 \mu \mathrm{l}\end{array}$ \\
\hline $\begin{array}{l}\text { Seperating Gel } 10 \% \\
\text { (12 ml; } 2 \text { Gels) }\end{array}$ & $\begin{array}{l}\text { Acrylamide } \\
4 x \text { Tris/SDS pH } 8.8 \\
\text { Aqua dest. } \\
10 \% \text { APS } \\
\text { TEMED }\end{array}$ & $\begin{array}{l}4 \mathrm{ml} \\
3 \mathrm{ml} \\
5 \mathrm{ml} \\
48 \mu \mathrm{l} \\
18 \mu \mathrm{L}\end{array}$ \\
\hline $\begin{array}{l}\text { Seperating Gel } \mathbf{1 5 \%} \\
\text { (12 ml; } 2 \text { Gels) }\end{array}$ & $\begin{array}{l}\text { Acrylamide } \\
4 x \text { Tris/SDS pH } 8.8 \\
\text { Aqua dest. } \\
10 \% \text { APS } \\
\text { TEMED }\end{array}$ & $\begin{array}{l}6 \mathrm{ml} \\
3 \mathrm{ml} \\
3 \mathrm{ml} \\
48 \mu \mathrm{l} \\
18 \mu \mathrm{L}\end{array}$ \\
\hline
\end{tabular}


Table 10 Solutions for PDE activity assay

\begin{tabular}{|c|c|c|}
\hline Buffer & Composition & \\
\hline Wash Buffer & $\begin{array}{l}\text { Tris } \\
\mathrm{pH}\end{array}$ & $\begin{array}{l}40 \mathrm{mM} \\
8.0(\mathrm{HCl})\end{array}$ \\
\hline Homogenation Buffer & $\begin{array}{l}\text { Wash Buffer } \\
\mathrm{MgCl}_{2} \\
\text { PhosStop } \\
\text { Protease Inhibitor } \\
\text { Cocktail }\end{array}$ & $\begin{array}{l}10 \mathrm{ml} \\
10 \mathrm{mM} \\
1 \text { tablet } \\
1 \text { tablet }\end{array}$ \\
\hline cAMP Stock Solution & cAMP & $1 \mathrm{mM}$ \\
\hline BSA Stock Solution & BSA & $10 \%(w / v)$ \\
\hline$\left[{ }^{3} \mathrm{H}\right]$ CAMP Stock Solution & {$\left[{ }^{3} \mathrm{H}\right] \mathrm{CAMP}$} & $1 \mathrm{mCi} / \mathrm{ml}$ \\
\hline $\begin{array}{l}\text { Ready to use } \\
\text { Reaction Buffer }\end{array}$ & $\begin{array}{l}\mathrm{MgCl}_{2} \\
\beta \text {-Mercaptoethanol } \\
\text { cAMP } \\
\text { BSA } \\
{\left[{ }^{3} \mathrm{H}\right] \text { cAMP }} \\
\text { in Wash Buffer }\end{array}$ & $\begin{array}{l}10 \mathrm{mM} \\
10 \mathrm{mM} \\
2 \mu \mathrm{M} \\
1.5 \%(\mathrm{w} / \mathrm{v}) \\
2.5 \mu \mathrm{l} / \mathrm{ml}\end{array}$ \\
\hline Stop Solution & $\begin{array}{l}\text { EDTA } \\
\mathrm{pH} \\
\text { in Wash Buffer }\end{array}$ & $\begin{array}{l}15 \mathrm{mM} \\
8.0(\mathrm{NaOH})\end{array}$ \\
\hline $\begin{array}{l}\text { Crotalus atrox } \\
\text { Snake Venom }\end{array}$ & Snake Venom & $1 \mathrm{mg} / \mathrm{ml}$ \\
\hline
\end{tabular}




\subsection{Methods}

\subsubsection{Cell culture and transfection}

HEK293A cells were cultivated at $37^{\circ} \mathrm{C}$ and $5 \% \mathrm{CO}_{2}$ in DMEM medium with $4.5 \mathrm{~g} / \mathrm{l}$ glucose, $10 \%$ FCS, $2 \mathrm{mM}$ L-glutamine, $100 \mathrm{U} / \mathrm{ml}$ penicillin and $100 \mu \mathrm{g} / \mathrm{ml}$ streptomycin.

For transfection of HEK293A cells with plasmid DNA, cells were plated on 6 well plates prepared with $24 \mathrm{~mm}$ round glass coverslips. After $24 \mathrm{~h}$, when the cells reached $60 \%$ confluency, the following precipitation mix was prepared under sterile conditions:

$\left.\begin{array}{lr}\mathrm{H}_{2} \mathrm{O} & 440 \mu \mathrm{l} \\ \begin{array}{l}10 \mu \mathrm{l} \\ 2.5 \mathrm{M} \mathrm{CaCl}_{2}\end{array} \\ \begin{array}{l}50 \mu \mathrm{l}\end{array}\end{array}\right\}$ mix

After 10 min incubation at RT, $166 \mu \mathrm{l}$ of the reaction mix were added dropwise to each well. $24 \mathrm{~h}$ after transfection, cells were used for FRET measurements.

\subsubsection{Generation of Epac1-PLN biosensors}

\subsubsection{Epac1-PLN (pcDNA3.0 vector)}

For the generation of the pcDNA3.0 Epac1-PLN construct, which was the basis for the following constructs used in this $\mathrm{PhD}$ thesis, the pcDNA3.0 vector containing the DNA sequence of the cytosolic cAMP sensor Epac1camps (Nikolaev et al., 2004) was double digested via Xbal/ Xhol. Then it was fused to the $5^{\prime}$-end of a full length rabbit PLN coding sequence

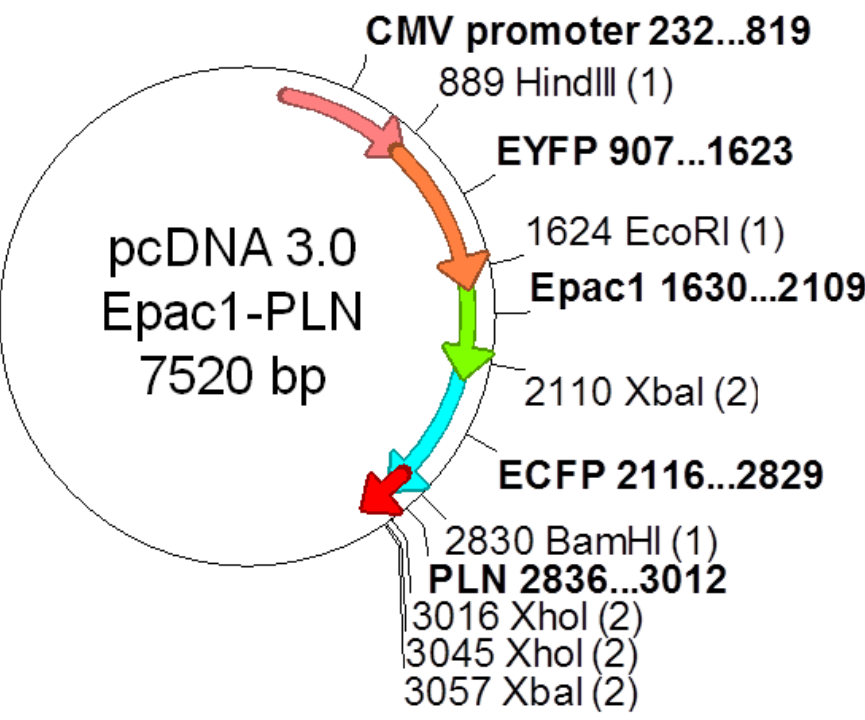

via a flexible linker encoding a GSMPLVDFFC amino acid motif. Therefore, CFP without the stop Figure 7. Plasmid map of the pcDNA3.0 Epac1-PLN construct with highlighted relevant restriction sites. The cytomegalovirus (CMV) promoter allows constitutive expression in mammalian cells. 
codon, the first insert, was PCR amplified (Primer: CFPXbalFor and CFPBamHIRev) and digested with $\mathrm{Xbal} / \mathrm{BamHI}$. The second insert was a full length rabbit PLN sequence that was kindly provided by Prof. Dr. med. Joachim Schmitt from Würzburg. The PLN sequence was amplified introducing a BamHI site including the linker sequence (Primer: PLBBamP4linHaseFor) and a Xhol site (Primer: PLBXhostHaseRev). See Figure 7 for plasmid map.

\subsubsection{Truncated Epac1-PLN (pcDNA3.0 vector)}

A PLN truncated version of Epac1-PLN was generated by amplifying a truncated PLN sequence (including glutamic acid at position 19 to leucine at position 52, E19-L52; Primer: TruncNhelFor, PLBXhostHaseRev) that was digested via Nhel and then fused to Epac1camps without a linker sequence. Apart from that, the cloning strategy was as for the full length Epac1-PLN.

The full length and truncated pcDNA3.0 Epac1-PLN constructs were already generated in Würzburg by Dr. Viacheslav Nikolaev.

\subsubsection{Epac1-PLN R279E (pcDNA3.0 vector)}

For some control experiments in HEK293A cells, a cAMP insensitive mutant of pcDNA3.0 Epac1-PLN was generated. Therefore, an Epac1-camps construct generated by Dr. Viacheslav Nikolaev in Würzburg was used, which contains a mutation in the Epac1 cAMP binding site (Arginine exchanged for Glutamic acid at position 297, R279E). The mutated R279E-Epac1 sequence was excised from this plasmid via EcoRI/ Xbal double digestion of $10 \mu \mathrm{g}$ plasmid DNA. Because pcDNA3.0 Epac1-PLN vector cannot be digested via EcoRI and Xbal without also losing the CFP and PLN sequence, CFP was extracted from $10 \mu \mathrm{g}$

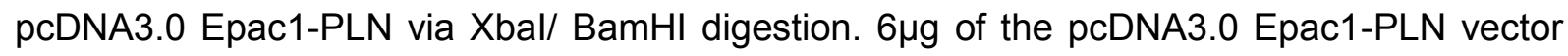
were digested with EcoRI/ BamHI to dispose of the CFP and Epac1 sequence. After purification of the digested fragments and vector on a $1 \%$ agarose gel, DNA was extracted using the Qiaquick Gel Extraction Kit. The digested pcDNA3.0 Epac1-PLN vector was eluted in $50 \mu$ l elution buffer and the CFP and mutated R279E-Epac1 fragments each were eluted in $25 \mu$ elution buffer. Ligation of the digested fragments and vector was performed in a reaction using $\mathrm{T} 4$ ligase overnight (o.n.) at $14^{\circ} \mathrm{C}$ : 


$\begin{array}{lr}\text { pcDNA3.0 Epac1-PLN ( 6300 bp) } & 1 \mu \mathrm{l} \\ \text { CFP }(\sim 700 \mathrm{bp}) & 6.5 \mu \mathrm{l} \\ \text { R279E Epac1 }(\sim 500 \mathrm{bp}) & 5 \mu \mathrm{l} \\ \text { T4 Ligase buffer } 10 \mathrm{x} & 1.5 \mu \mathrm{l} \\ \text { T4 Ligase } & 1 \mu \mathrm{l}\end{array}$

\subsubsection{Epac1-PLN ( $\alpha-M H C$ vector)}

To generate Epac1-PLN transgenic sensor mice in this thesis, the pcDNA3.0 Epac1-PLN construct was digested via BamHI restriction sites and subcloned into a vector containing the a-myosin heavy chain ( $\alpha-\mathrm{MHC}$ ) promoter for cardiac specific expression (Nikolaev et al., 2006). To delete the dispensable 12 bp sequence between the $\alpha-M H C$ promoter and the sensor, the construct was digested via Kpnl. Sequencing analysis of this construct revealed several amino acid replacements in the YFP region like Asparagine replaced by Isoleucine (N147I), Methionine by Threonine (M154T), Valine by Alanine (V164A) and Asparagine replaced by Histidine $(\mathrm{N} 165 \mathrm{H})$. To exclude these mutations, YFP from the already published HCN2-camps cAMP sensor (Nikolaev et al., 2006) was excised via EcoRI and Kpnl digestion of $10 \mu \mathrm{g}$ plasmid DNA. The Epac1-CFP-PLN sequence part of the $\alpha-M H C$ Epac1-PLN was not altered according to sequencing analysis and was extracted from this plasmid via EcoRI and Xhol double digestion also using $10 \mu \mathrm{g}$ of plasmid DNA. $6 \mu \mathrm{g}$ of the $\alpha-\mathrm{MHC}$ Epac1-PLN vector were digested with $\mathrm{Kpnl}$ and $\mathrm{Xhol}$ to dispose of the YFP mutated Epac1-PLN sensor sequence. Fragment purification was performed as for R279E-Epac1. Ligation of the digested fragments and vector was performed in a reaction using T4 ligase o.n. at $14^{\circ} \mathrm{C}$ :

$\begin{array}{lr}\text { a-MHC Epac1-PLN ( 8800 bp) } & 1 \mu \mathrm{l} \\ \text { YFP ( 700 bp) } & 4.5 \mu \mathrm{l} \\ \text { Epac-CFP-PLN ( 1400 bp) } & 7 \mu \mathrm{l} \\ \text { T4 Ligase buffer 10x } & 1.5 \mu \mathrm{l} \\ \text { T4 Ligase } & 1 \mu \mathrm{l}\end{array}$

a-MHC Epac1-PLN construct was used for the generation of TG mice (Nikolaev et al., 2006).

\subsubsection{Transformation of E.coli for plasmid amplification}

To amplify plasmid DNA, the ligation mix was introduced into E. coli TOP10 (competence $1 \times 10^{9} \mathrm{cfu} / \mu \mathrm{g}$ DNA) using the following reaction mix: 


$\begin{array}{lr}\text { E.coli TOP10 } & 100 \mu \mathrm{l} \\ \text { Ligation Mix } & 15 \mu \mathrm{l} \\ \mathrm{H}_{2} \mathrm{O} & 65 \mu \mathrm{l} \\ 5 x \text { KCM Buffer } & 20 \mu \mathrm{l}\end{array}$

The reaction mix was incubated on ice for $20 \mathrm{~min}$ followed by 10 min incubation at RT. To allow bacterial growth, $1 \mathrm{ml}$ of LB medium without antibiotics was added to the mix and the solution was incubated for $50 \mathrm{~min}$ at $37^{\circ} \mathrm{C}$ in a ThermoMix (700 rpm). Afterwards, bacteria were plated on ampicillin selective LB- medium plates. Colonies were grown o.n. at $37^{\circ} \mathrm{C}$ and single colonies were harvested and incubated in $3 \mathrm{ml} \mathrm{LB}$ medium with ampicillin o.n. at $37^{\circ} \mathrm{C}$. Plasmid DNA was extracted using the Qiagen Plasmid Mini Kit with subsequent restriction analysis. Positive colonies were grown in $200 \mathrm{ml}$ LB medium with ampicillin over night at $37^{\circ} \mathrm{C}$ and plasmid DNA was extracted using the Qiagen Plasmid Midi Kit. Plasmid DNA concentration was measured using the Nanodrop device, and plasmid DNA sequencing was performed at Eurofins.

\subsubsection{Generation of Epac1-PLN adenovirus}

Epac1-PLN adenovirus generation was performed using the Invitrogen Gateway ${ }^{\circledR}$ Cloning system.

To amplify the Epac1-PLN sensor sequence, pcDNA3.0 Epac1-PLN was used as PCR template:

$\begin{array}{lr}\mathrm{H}_{2} \mathrm{O} & 81 \mu \mathrm{l} \\ \text { 10x Pfu buffer } & 10 \mu \mathrm{l} \\ \text { dNTPs } 10 \mathrm{mM} & 2 \mu \mathrm{l} \\ \text { pcDNA3attBFor }(10 \mathrm{pmol} / \mu \mathrm{l}) & 2.5 \mu \mathrm{l} \\ \text { pcDNA3attBRev }(10 \mathrm{pmol} / \mu \mathrm{l}) & 2.5 \mu \mathrm{l} \\ \text { pcDNA3 Epac1 PLN }(200 \mathrm{ng}) & 1 \mu \mathrm{l} \\ \text { Pfu DNA Polymerase } & 1 \mu \mathrm{l}\end{array}$

The PCR reaction was as follows:

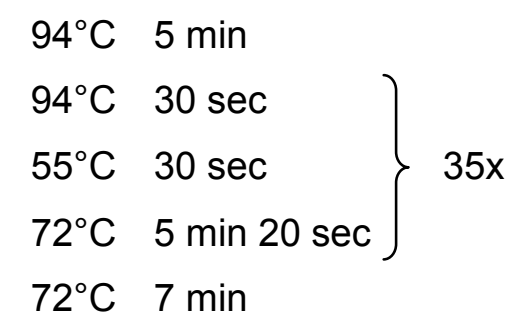


The PCR product was purified on a $1 \%$ agarose gel in TAE buffer stained with ethidium bromide $(0.5 \mu \mathrm{g} / \mathrm{ml})$. The band was excised under UV light using a scalpel and extracted using the Qiaquick Gel extraction Kit. DNA concentration was measured using the Nanodrop device. To generate the entry clone, in vitro recombination of the PCR product and the Donor vector Gateway ${ }^{\circledR}$ pDONR ${ }^{\mathrm{TM}} 221$ was performed using the Gateway ${ }^{\circledR}$ BP Clonase ${ }^{\mathrm{TM}}$ II Enzyme Mix. The recombinant entry clone was amplified in One Shot ${ }^{\circledR}$ OmniMax2T1 bacteria o.n. at $37^{\circ} \mathrm{C}$ on a kanamycin selective LB- medium plate. Single colonies were harvested and grown as described in section 2.2.2 and analysed via EcoRI/ Xhol double digestion. To generate the virus expression clone, in vitro recombination between the entry clone and the destination vector $\mathrm{pAd} / \mathrm{CMV} / \mathrm{V} 5-\mathrm{DEST} \mathrm{T}^{\mathrm{TM}}$ was performed using the Gateway ${ }^{\circledR}$ LR Clonase ${ }^{\mathrm{TM}}$ II Enzyme Mix. The recombinant Epac1-PLN adenoviral vector DNA was amplified and analysed as described for the entry clone but using ampicillin instead of kanamycin selective LB medium plates. All procedures were performed regarding manufacturer's instructions.

Before HEK293A transfection, Epac1-PLN adenoviral vector DNA was linearized as follows:

$\begin{array}{lrl}\text { DNA } & 5 \mu \mathrm{g} \\ \text { 10x buffer NEB1 } & 2.5 \mu \mathrm{l} \\ \text { 100x BSA } & 0.25 \mu \mathrm{l} \\ \text { Pacl } & 2 \mu \mathrm{l} \\ \mathrm{H}_{2} \mathrm{O} & \text { ad } 25 \mu \mathrm{l}\end{array}$

For ethanol precipitation of the digested vector, $70 \mu \mathrm{l}$ of ice-cold $100 \%$ ethanol and $10 \mu \mathrm{l} 3 \mathrm{M}$ sodiumacetate solution were added to the digestion mix and incubated for $30 \mathrm{~min}$ at $-20^{\circ} \mathrm{C}$. After centrifugation for $10 \mathrm{~min}$ at $13.300 \mathrm{rpm}$, the DNA pellet was washed with $500 \mu \mathrm{l}$ ice-cold $75 \%$ ethanol and centrifuged again for $5 \mathrm{~min}$ at $13.000 \mathrm{rpm}$. The DNA pellet was resuspended in $10 \mu \mathrm{L} \mathrm{H}_{2} \mathrm{O}$ and used for transfection of a $10 \mathrm{~cm}$ plate with HEK-293A (80\% confluency) via lipofectamine ${ }^{\circledR} 2000$ reagent in OPTI-MEM ${ }^{\circledR}$ regarding manufacturer's instructions. After 7-14 days, virus production within the HEK293A cells was completed. The virus containing supernatant was collected and used for further HEK293A transduction and virus amplification in Iscove Basal Medium (5\% FCS, $1 \%$ antibiotic, antimycotic). The virus was concentrated via ultracentrifugation of the lysed HEK293A cell supernatant at 40.000 rpm o.n. at $16^{\circ} \mathrm{C}$ in a cesium chloride gradient. The virus band was collected using a 21 gauge needle and dialyzed in $1 \mathrm{x}$ sucrose buffer. For physical virus concentration OD260nm was measured. The virus solution was then diluted 1:100 with sucrose buffer containing 10 $\%$ glycerol and aliquots were stored at $-80^{\circ} \mathrm{C}$. Biological virus activity (expressed as plaque forming units per $\mathrm{ml}$ ) was assessed via Plaque assay as previously described (Cooper, 1961) in HEK293A cells covered with $1.5 \%$ plaque GP agarose. Adult rat ventricular cardiomyocytes were isolated and provided by Gudrun Müller from the Department of 
Cardiology. Cells were transduced with Epac1-PLN virus (MOI 300) and used for analysis 48 $\mathrm{h}$ after transduction.

\subsubsection{Generation of Epac1-PLN transgenic mice}

For microinjections, the generated Epac1-PLN construct was linearized using Notl digestion overnight at $37^{\circ} \mathrm{C}$ as follows:

$\begin{array}{lr}\text { DNA } & 50 \mu \mathrm{g} \\ \text { Notl } & 10 \mu \mathrm{l} \\ \text { Buffer4 } & 20 \mu \mathrm{l} \\ \text { 100xBSA } & 2 \mu \mathrm{l} \\ \mathrm{H}_{2} \mathrm{O} \quad \text { ad } 200 \mu \mathrm{l}\end{array}$

The linearized construct was purified on a $1 \%$ agarose gel in TAE buffer stained with ethidium bromide $(0.5 \mu \mathrm{g} / \mathrm{ml})$, extracted using the Qiaquick Gel extraction Kit and eluted in $100 \mu \mathrm{l}$ sterile TE buffer. DNA concentration was measured using the Nanodrop device. After filtration of the linearized and purified Epac1-PLN construct through a $2 \mu \mathrm{m}$ filter under sterile conditions, the DNA was transferred into a sterile dialysis chamber. Dialysis was performed in $500 \mathrm{ml}$ TE buffer which was exchanged every 4 hours (overall 2 I TE buffer). The dialysis chamber was unloaded under sterile conditions and DNA concentration was measured using the Nanodrop device. A concentration of 20-40 $\mathrm{ng} / \mu \mathrm{l}$ was considered sufficient for microinjections. The pronuclear injection of FVB/N mice with the Epac1-PLN construct was performed by the Max Planck Institute of Experimental Medicine as previously described (Buitrago et al., 2005).

\subsubsection{Genotyping PCR}

The Founder mice and their resulting heterozygote offspring were genotyped by a standard PCR. Tail biopsies were digested overnight in $200 \mu \mathrm{l}$ DirectPCR-Tail buffer including Proteinase $\mathrm{K} 500 \mu \mathrm{g} / \mathrm{ml}$ at $55^{\circ} \mathrm{C}$ and $1000 \mathrm{rpm}$ in a ThermoMix. The reaction was terminated by incubation at $85^{\circ} \mathrm{C}$ for $45 \mathrm{~min}$. After the lysates cooled down, they were used for the following PCR reaction: 


$\begin{array}{lr}\text { DNA } & 0.5 \mu \mathrm{l} \\ \mathrm{H}_{2} \mathrm{O} & 14.7 \mu \mathrm{l} \\ \text { 5x GoTaq buffer } & 4.0 \mu \mathrm{l} \\ \text { dNTPs } 10 \mathrm{mM} & 0.5 \mu \mathrm{l} \\ \text { MHCseqFor }(100 \mathrm{pmol} / \mu \mathrm{l}) & 0.05 \mu \mathrm{l} \\ \text { YFPnewRev }(100 \mathrm{pmol} / \mu \mathrm{l}) & 0.05 \mu \mathrm{l} \\ \text { GoTaq Polymerase } & 0.2 \mu \mathrm{l}\end{array}$

The PCR reaction was as follows:

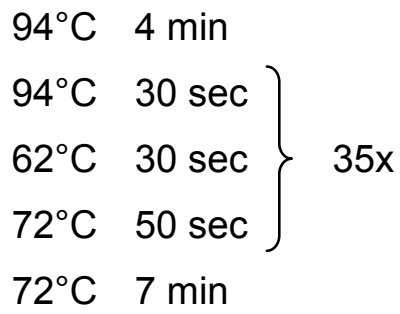

The PCR reaction was mixed with $6 x$ loading dye and analysed on a $2 \%$ agarose gel in TAE buffer stained with ethidium bromide $(0.5 \mu \mathrm{g} / \mathrm{ml})$. A $100 \mathrm{bp}$ DNA Ladder (Quickload) was used as DNA marker. Results were documented using the Multilmage Light Cabinet gel documentation system. Positive male founder mice were mated with WT FVB/NRj females to produce heterozygous offspring.

\subsubsection{Transverse aortic constriction (TAC)}

All animal experiments were performed in accordance with institutional and governmental guidelines. Female FVB/N mice aged 8-12 weeks were randomized into sham or TAC groups. 3 days before surgery, mice received analgesic therapy with metamizole. Right before surgery, mice were anesthetized using $2 \%$ isoflurane (Forene®) in pure oxygen. A suprasternal incision was made, and the aortic arch was visualized using a binocular operating stereoscope. TAC interventions used a spacer-defined (26-gauge) constriction fixed by a 6-0 polyviolene suture between the first and second trunk of the aortic arch (Hu et al., 2003). For sham, the aorta was exposed as for TAC but not constricted. 3 days later, Doppler velocity was measured using a $20 \mathrm{MHz}$ probe to quantify the pressure gradient across the TAC/ sham region by transthoracic echocardiography. A pressure gradient of at least $80 \mathrm{mmHg}$ was defined as appropriate for the following experiments. During 1 week after surgery, mice received analgesic therapy with metamizole and animal health status was checked every day. 8 weeks after surgery, mice were analysed by echocardiography and hearts were harvested for ventricular cardiomyocyte isolation or histology. Surgeries were 
performed by Julia Steinbrecher (AG Lehnart, Cardiology and Pneumology, University Medical Centre Göttingen).

\subsubsection{Echocardiography}

Heart function of untreated, sham or TAC operated mice was characterized by echocardiography (Vevo 2100) using a $30 \mathrm{~Hz}$ transducer (MS-400 MicroScan Transducer). Untreated animals received echocardiography at the age of 3 and 6 months, whereas TAC and $\mathrm{Ml}$ treated animals were characterized 8 and 12 weeks after surgery, respectively. The echocardiographic results were used to analyse the animal heart morphology and function: septum thickness, left ventricular enddiastolic diameter (LVEDD), left ventricular endsystolic diameter (LVESD), enddiastolic volume (EDV), endsystolic volume (ESV), heart frequency and heart weight to body weight ratio. These parameters were used to calculate characteristic variables such as ejection fraction (EF) and fractional shortening (FS). Echocardiography and data analysis were performed by the SFB service team as well was by Kirsten Koschel and Sabrina Wollborn (Cardiology and Pneumology, University Medical Centre Göttingen) in a blind test for the untreated and treated animals.

\subsubsection{Heart weight to body weight and heart weight to tibia length calculation}

Mice were euthanized and death was guaranteed by cervical dislocation. Mouse weight was determined before the heart was cut out and transferred into a petri dish filled with ice-cold PBS. Here, the aorta was connected to a $21 \mathrm{G}$ cannula filled with PBS to perfuse the heart until blood free. Heart weight was estimated and hearts were shock frozen for biochemical analysis. Tibia length was calculated using an electronic ruler.

\subsubsection{Cardiomyocyte isolation via Langendorff perfusion}

Adult ventricular cardiomyocytes were isolated via the Langendorff perfusion as previously described (Borner et al., 2011). Mice were euthanized and death was guaranteed by cervical dislocation. The heart was harvested by cutting the aorta and then quickly transferred into a petri dish filled with ice-cold PBS. Here, the aorta was connected to a $21 \mathrm{G}$ cannula filled with perfusion buffer. The heart was Langendorff perfused at $37^{\circ} \mathrm{C}$ with perfusion buffer (flow rate $3.5 \mathrm{ml} / \mathrm{min}$ ) for $3 \mathrm{~min}$ followed by perfusion with $30 \mathrm{~mL}$ digestion buffer. Afterwards, the atria were carefully excised and discarded, whereas the digested ventricles were dissected for 30 
$\mathrm{sec}$ in $2.5 \mathrm{~mL}$ digestion buffer. To stop the digestion, $2.5 \mathrm{~mL}$ Stop buffer I were added to the cell suspension, which was then homogenized using a $1 \mathrm{~mL}$ syringe without a needle for 3 min. 10 min after sedimentation, the cardiomyocyte pellet was transferred into Stop buffer II for recalcification. The recalcification process was as follows, with 4 min adaptation time between each step:

$\begin{array}{lllr}50 \mu \mathrm{L} \mathrm{CaCl}_{2} & 10 \mathrm{mM} & \text { final concentration } & 62 \mu \mathrm{M} \\ 50 \mu \mathrm{L} \mathrm{CaCl}_{2} & 10 \mathrm{mM} & \text { final concentration } & 112 \mu \mathrm{M} \\ 100 \mu \mathrm{L} \mathrm{CaCl}_{2} & 10 \mathrm{mM} & \text { final concentration } & 212 \mu \mathrm{M} \\ 30 \mu \mathrm{L} \mathrm{CaCl}_{2} & 100 \mathrm{mM} & \text { final concentration } & 500 \mu \mathrm{M} \\ 50 \mu \mathrm{L} \mathrm{CaCl}_{2} & 100 \mathrm{mM} & \text { final concentration } & 1000 \mu \mathrm{M}\end{array}$

After recalcification, the cardiomyocytes were plated onto laminin (Sigma) coated round glass coverslides (24 mm, Thermo Sientific) and incubated at $37^{\circ} \mathrm{C}$ and $5 \% \mathrm{CO}_{2}$ until use. For immunoblot analysis, cardiomyocyte isolation was performed as described but with BSA free Stop buffer II.

Lagendorff isolated rat cardiomyocytes were provided by Gudrun Müller from the cardiology department.

\subsubsection{FRET measurements in cardiomyocytes and data analysis}

Laminin coated coverslides with isolated cardiomyocytes were mounted in the imaging chamber. The cells were washed once with $300 \mu \mathrm{L}$ of FRET buffer and $300 \mu \mathrm{L}$ of fresh FRET buffer were added to the chamber. The different compounds to be used in the following imaging experiments were diluted in FRET buffer. For FRET measurements in paced cardiomyocytes, FRET buffer was replaced with Tyrode for $\mathrm{Ca}^{2+}$ measurements. FRET measurements were performed using an inverted fluorescent microscope and ImageJ software (Sprenger et al., 2012). CFP, the FRET donor, was excited at $440 \mathrm{~nm}$ using a CoolLED single-wavelength light emitting diode. The emitted light from the sample was split into CFP and YFP signals using a dual view and detected via a CCD camera (Figure 8). Cardiomyocytes with optimal sensor expression were selected using live fluorescent light. To avoid photobleaching of the FRET sensor, the fluorescent light was switched off immediately after finding an appropriate cell. An exposure time of $10 \mathrm{~ms}$ usually led to good signal-tonoise ratio and images were acquired in CFP and YFP emission channels every $5 \mathrm{~s}$. $300 \mu \mathrm{L}$ of the desired compound solution were transferred into the chamber as soon as the FRET ratio reached a stable baseline. 

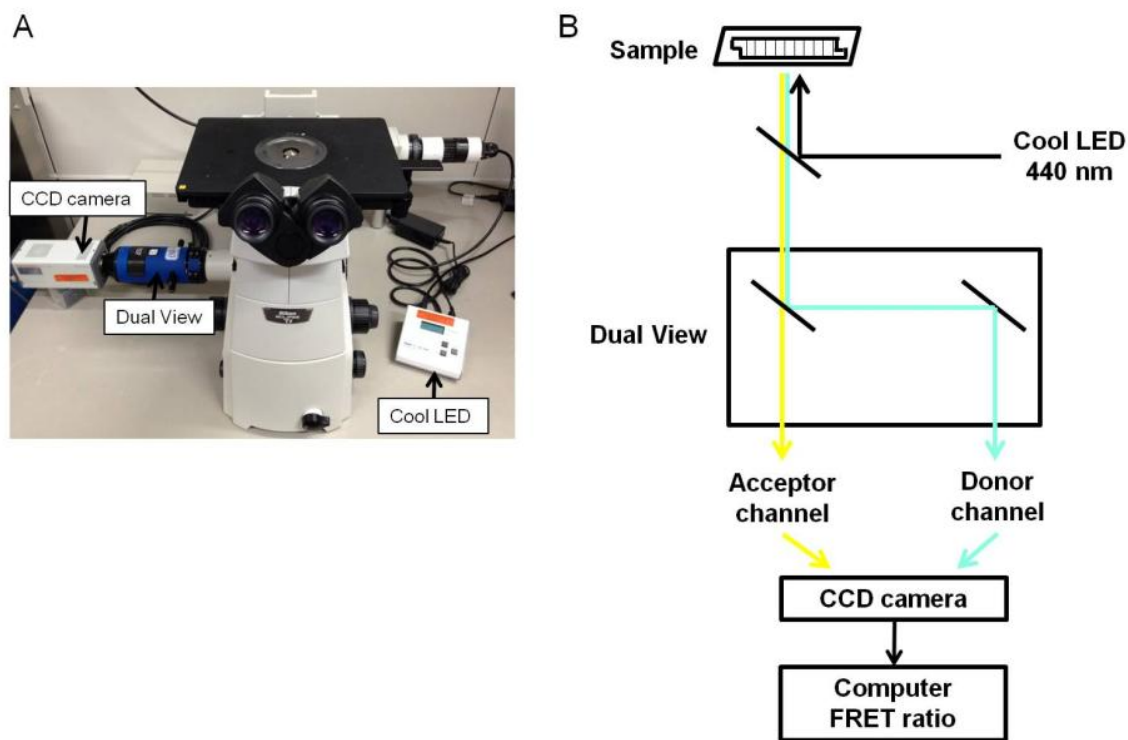

Figure 8. FRET microscope setup. (A) Overview of the FRET microscope setup. (B) The Cool LED light source is used for CFP excitation at $440 \mathrm{~nm}$ within the transgenic cardiomyocytes. The Dual View splits the emitted sample fluorescence into an acceptor channel (YFP) and a donor channel (CFP). Both signals are detected via a CCD camera and transferred to a computer that calculates the FRET ratio using ImageJ software.

FRET experiments in paced cardiomyocytes were performed at $1 \mathrm{~Hz}$ field stimulation and 20$30 \mathrm{~V}$ but under the same conditions as described before.

FRET imaging data were analysed offline using Origin 8.5 software and Excel. The single CFP and YFP intensities from each experiment were measured using ImageJ and copypasted into an Excel or Origin spreadsheet to calculate the corrected FRET ratio. We corrected for the bleedthrough of the donor fluorescence (CFP) into the acceptor (YFP) channel, in our case

Ratio $=(\mathrm{YFP}-0.63 \times \mathrm{CFP}) / \mathrm{CFP}$.

For more detailed description of the microscope setup and FRET analysis, please refer to the following literature (Sprenger et al., 2012).

\subsubsection{Quantitative real time PCR}

Heart tissue RNA was isolated using the RNeasy purification Kit, and subsequent cDNA synthesis was performed using the iScript cDNA Synthesis Kit according to manufacturer instructions. Standard genes for GAPDH (primer GAPDHFor and GAPDHRev) and phospholamban (primer PLNFor and PLNRev) were amplified using the following reaction mix: 


$\begin{array}{lcc}\text { wt cDNA 1:10 } & 2.5 \mu \mathrm{l} \\ \mathrm{H}_{2} \mathrm{O} & 33.125 \mu \mathrm{l} \\ \text { 5x GoTaq buffer } & 10.0 \mu \mathrm{l} \\ \text { dNTPs } 10 \mathrm{mM} & 1.25 \mu \mathrm{l} \\ \text { PrimerFor }(10 \mathrm{pmol} / \mu \mathrm{l}) & 1.25 \mu \mathrm{l} \\ \text { PrimerRev }(10 \mathrm{pmol} / \mu \mathrm{l}) & 1.25 \mu \mathrm{l} \\ \text { GoTaq Polymerase } & 0.2 \mu \mathrm{l}\end{array}$

and PCR protocol:

$\left.\begin{array}{ll}94^{\circ} \mathrm{C} & 4 \mathrm{~min} \\ 94^{\circ} \mathrm{C} & 30 \mathrm{sec} \\ 55^{\circ} \mathrm{C} & 30 \mathrm{sec} \\ 72^{\circ} \mathrm{C} & 40 \mathrm{sec} \\ 72^{\circ} \mathrm{C} & 7 \mathrm{~min}\end{array}\right\} 35 \mathrm{x}$

Standards were purified using the Qiagen PCR Kit according to manufacturer instructions and eluted in $30 \mu \mathrm{L}$ of buffer EB. Purity of the standards was assured by agarose gel electrophoresis of $2 \mu \mathrm{L}$ standard eluate. Standard dilutions ranging from $100 \mathrm{pg} / \mu \mathrm{l}$ to $1 \mathrm{ag} / \mu \mathrm{L}$ were prepared in RNase free $\mathrm{H}_{2} \mathrm{O}$. Quantitative real time PCR was conducted using the SYBR Green Super Mix for $\mathrm{Q} Q$ and the iCycler according to the following protocol:

$\left.\begin{array}{ll}95^{\circ} \mathrm{C} & 3 \mathrm{~min} \\ 95^{\circ} \mathrm{C} & 15 \mathrm{sec} \\ 55^{\circ} \mathrm{C} & 10 \mathrm{sec} \\ 72^{\circ} \mathrm{C} & 15 \mathrm{sec}\end{array}\right\} 40 \mathrm{x}$

Data were analysed using BioRad iQ5 software, and phospholamban mRNA levels were normalized to GAPDH mRNA levels of the same cDNA sample.

\subsubsection{Fractionation and immunoblot analysis}

To harvest the hearts for fractionation analysis, mice were euthanized, the thorax was cut open and the heart was excised at the aorta and quickly transferred into a petri dish filled with ice-cold PBS. Here, the aorta was connected to a $21 \mathrm{G}$ cannula filled with ice-cold PBS. The heart was perfused with ice-cold PBS until blood free and then shock frozen. Heart 
tissue was processed using the Qproteome Cell Compartment Kit (Qiagen) according to manufacturer protocol and cytosolic and membrane fractions were subjected to $10-15 \%$ SDS-PAGE and to immunoblotting.

To analyse phospholamban phosphorylation under basal and various stimulated conditions, freshly isolated cardiomyocytes were sedimented in Stop buffer II without BSA and then incubated either with vehicle, $100 \mathrm{nM}$ ISO, $100 \mathrm{nM}$ BAY 60-7550, $10 \mu \mathrm{M}$ cilostamide, $10 \mu \mathrm{M}$ rolipram or $100 \mu \mathrm{M}$ IBMX for $15 \mathrm{~min}$ at $37^{\circ} \mathrm{C}$. To analyse specific protein compositions under healthy and diseased conditions, cardiomyocytes from untreated, sham and TAC animals were isolated and collected.

Cell pellets were shock frozen, homogenized in homogenation buffer and quantified using BCA Protein Assay. 10-30 $\mu \mathrm{g}$ protein were diluted in homogenation buffer including 3xSDS stop buffer and loaded on 8-15\% SDS-PAGE modified from Laemmli (Laemmli, 1970), depending on the protein size, and transferred onto a nitrocellulose membrane according to Towbin (Towbin et al., 1979). Proteins were detected using the antibodies listed in Table 1 and 2. Band densitometry analysis was performed using ImageJ software.

\subsubsection{Histology and morphometric analysis}

For histology and morphometric analysis, mice were euthanized, the thorax was cut open and the heart was excised at the aorta and quickly transferred into a chamber filled with icecold PBS. Here, the aorta was connected to a $21 \mathrm{G}$ cannula filled with ice-cold PBS to perfuse the heart until blood free. The heart was fixed in $4 \%$ Roti Histofix at $4^{\circ} \mathrm{C}$ overnight. The fixed hearts were paraffin embedded and $5 \mu \mathrm{m}$ heart cross sections were generated at a Microtom (Leica RM 2165). For the following applications, paraffin cross sections were dewaxed in xylol (20 min twice) and rehydrated with 6 subsequent decreasing ethanol series (100\% - $25 \%, 5$ min each step) and with Aqua dest.

The hematoxylin-eosin stain of WT and TG heart sections was performed in the pathology department of the University Medical Center Göttingen using a standard automated procedure. Cell nuclei were stained thrice with hematoxylin ( 2 min each), and after 2 min washing the cytosol was stained twice using eosin (2 min each). After rehydration and mounting, heart sections were analysed using a Stemi 2000-C microscope binocular with associated AxioCam ICc1 and Axio Vision Software.

To determine cardiomyocyte dimensions, WT and TG heart cross-sections were incubated with Wheat Germ Agglutinin (WGA, $75 \mu \mathrm{g} / \mu \mathrm{l}$ ) for $30 \mathrm{~min}$ in the dark, washed with PBS thrice, mounted with VectaShield and observed under a Axiovert 200 microscope. AxioVision software was used for image acquisition and cell diameters of 100 cells from each heart section were measured and analysed using ImageJ. 
Immunostaining of TG Epac1-PLN heart cross sections was performed after heat induced epitope retrieval in citrate buffer (dilution 1:10 in Aqua dest.). After rehydration, heart sections were blocked wit $4 \%$ BSA in PBS for $1 \mathrm{~h}$ at $37^{\circ} \mathrm{C}$. Primary antibodies for SERCA2a and GFP (for Epac1-PLN detection) were used. The secondary antibodies were Alexa Fluor 633 and Alexa Fluor 514, respectively. For antibody dilution see Table 1 and 2. Antibody incubation was $1 \mathrm{~h}$ at $37^{\circ} \mathrm{C}$. After rehydration, the stained heart sections were mounted with VectaShield, and image acquisition was performed using confocal microscopy.

\subsubsection{Confocal Microscopy}

To analyse immunostained Epac1-PLN heart cross sections, confocal microscopy was performed using Zeiss LSM 710 NLO microscope equipped with a Plan-Apochromat x63/1.40 oil-immersion objective. Images were acquired for Alexa Fluor 514 (514 nm diode laser excitation) and Alexa Fluor 633 (633 nm diode laser excitation). ZEN 2010 software and Microsoft Office Picture Manager were used for image analysis.

\subsubsection{Calcium transients of single cardiomyocytes}

Freshly isolated cardiomyocytes were plated onto laminin coated glass coverslides and incubated with $1 \mu \mathrm{M}$ Fura2-AM (Caffeine experiments) or $1 \mathrm{mM}$ Fluo-3 $\left(\mathrm{Ca}^{2+}\right.$ decay estimation) for $15 \mathrm{~min}$ at $37^{\circ} \mathrm{C}$ in Tyrode solution for $\mathrm{Ca}^{2+}$ measurements. After washing the cells thrice with Tyrode solution, myocytes were field stimulated at $1 \mathrm{~Hz}$ and $20-30 \mathrm{~V}$ until steady state was achieved, and then $\mathrm{Ca}^{2+}$ transients were recorded for $60 \mathrm{sec}$ using the IonOptix system. For sarcoplasmic reticulum $\mathrm{Ca}^{2+}$ content estimation, $10 \mathrm{mM}$ caffeine was added to the resting cardiomyocytes. To analyse changes in $\mathrm{Ca}^{2+}$ transients under beta adrenergic stimulation, cardiomyocytes were treated with $100 \mathrm{nM}$ isoprenaline. $F / F_{0}$ values in Fura2-AM experiments were calculated from the ratios of $510 \mathrm{~nm}$ emission light measured at 340 and $380 \mathrm{~nm}$ excitations. Fluo3-AM loaded cardiomyocytes were excited at $506 \mathrm{~nm}$ and emission was measured at $526 \mathrm{~nm}$. Data were analysed using the lonWizard software.

\subsubsection{PDE activity assay}

Freshly isolated cardiomyocytes were lysed and processed for in vitro measurement of cAMP-PDE hydrolysing activity following the standard method by Thompson and Appleman in presence of $1 \mu \mathrm{M}$ cAMP substrate, as previously described (Thompson and Appleman, 
1971). After sedimentation of the isolated cardiomyocytes in Stop buffer I, cells were washed with washing buffer and lysed in $500 \mu \mathrm{L}$ homogenation buffer. Proteins were quantified using BCA Protein Assay. $30 \mu \mathrm{g}$ protein were set to a total volume of $200 \mu \mathrm{l}$ homogenation buffer containing PDE inhibitors. Contributions of individual PDE families were calculated from the effects of $100 \mathrm{nM}$ BAY 60-7550 (PDE2), $10 \mu \mathrm{M}$ cilostamide (PDE3), $10 \mu \mathrm{M}$ rolipram (PDE4), and $100 \mu \mathrm{M}$ IBMX (unselective PDE inhibitor). For maximal cAMP breakdown, each sample was incubated with $200 \mu \mathrm{L}$ reaction buffer for $10 \mathrm{~min}$ at $33^{\circ} \mathrm{C}$. Reaction was terminated after addition of $200 \mu \mathrm{L}$ Stop solution for $1 \mathrm{~min}$ at $95^{\circ} \mathrm{C}$. The samples were incubated with $50 \mu \mathrm{g}$ of Crotalus atrox snake venom for $20 \mathrm{~min}$ at $33^{\circ} \mathrm{C}$ to hydrolyse the $5^{\prime \prime}-\mathrm{AMP}$. The samples were

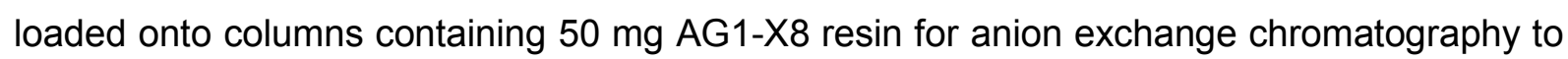
separate the adenosine. Quantification was performed by scintillation counting (MicroBeta ${ }^{2}$ ) in 96 well format (96 well plate for MicroBeta). Data were collected with the MicroBeta ${ }^{2}$ Windows Workstation.

\subsubsection{Statistics}

Echocardiographic, morphometric, real time PCR, immunoblot, lonoptix and FRET data were analysed using the Origin Pro 8.5 software (OriginLab Corporation, Northhampton, MA). Normal distribution was tested by the Kolmogorov-Smirnov test, and differences between the groups were analysed using one-way ANOVA or Mann-Whitney followed by Bonferroni"s post-hoc test as appropriate at the significance level of 0.05 . 


\section{Results}

\subsection{Generation of Epac1-PLN construct}

In the first experiment, the previously generated pcDNA3.0 based Epac1-PLN FRET sensor constructs were tested regarding their FRET efficiency in transiently transfected HEK 293A cells. The truncated Epac1-PLN sensor ( $\triangle$ PLN, Figure 9A) showed only relatively small changes in FRET upon $\beta$-adrenergic stimulation with isoprenaline (ISO) (Figure 9 B,C). On the other hand, the full length Epac1-PLN construct (Figure 9A) showed significantly larger FRET responses to $\beta$-adrenergic stimulation (Figure 9 B,C). Therefore, the full length Epac1PLN construct was used in further experiments and for the generation of TG animals.

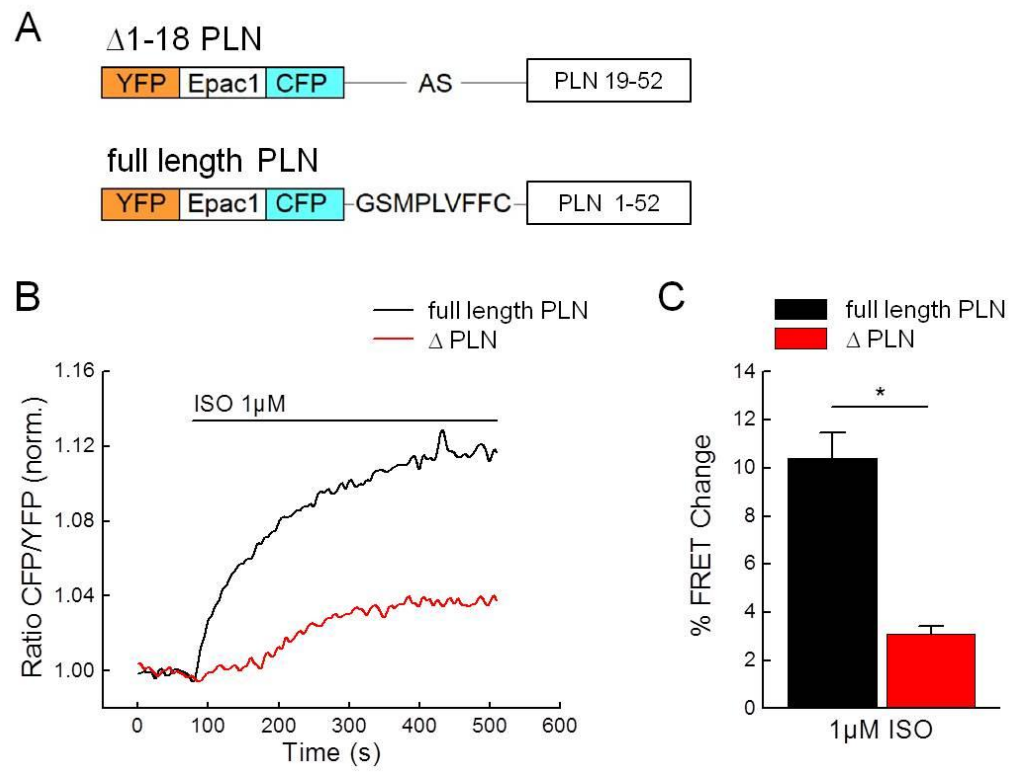

Figure 9. FRET responses of the full length PLN and truncated Epac1-PLN sensors. (A) Cartoon of the truncated $\triangle$ PLN and the full length PLN FRET sensors. (B) Responses to cAMP stimulation with $1 \mu \mathrm{M}$ isoproterenol (ISO) in transiently transfected HEK293A cells show higher signal amplitude for the full-length PLN construct. Quantification is in (C), shown are means \pm SE, $n=8-9$ cells per group. * - significant difference at $p<0.05$.

PLN is a phosphorylation target for PKA. To exclude that FRET changes in the Epac1-PLN construct are due to PKA modification of the sensor PLN, HEK cells were either transfected with full length PLN or the cAMP insensitive Epac1-PLN R279E mutant (Figure 10 A). Epac1PLN expressing cells showed the same FRET change after ISO stimulation independently of the presence of PKA inhibitor H-89. Besides, no FRET change was detected in the Epac1PLN R279E expressing cells (Figure $10 \mathrm{~B}, \mathrm{C}$ ). These results show that the FRET change in 
the Epac1-PLN sensor truly represents cAMP binding to the Epac1-PLN construct rather than PKA associated sensor PLN phosphorylation artefacts.
A Epac1-PLN
\begin{tabular}{|l|l|l|l|l|}
\hline YFP & Epac1 & CFP & GSMPLVFFC & PLN 1-52 \\
\hline
\end{tabular}
Epac1-PLN R279E
\begin{tabular}{|l|l|l|}
\hline YFP & R279E & CFP \\
\hline
\end{tabular}
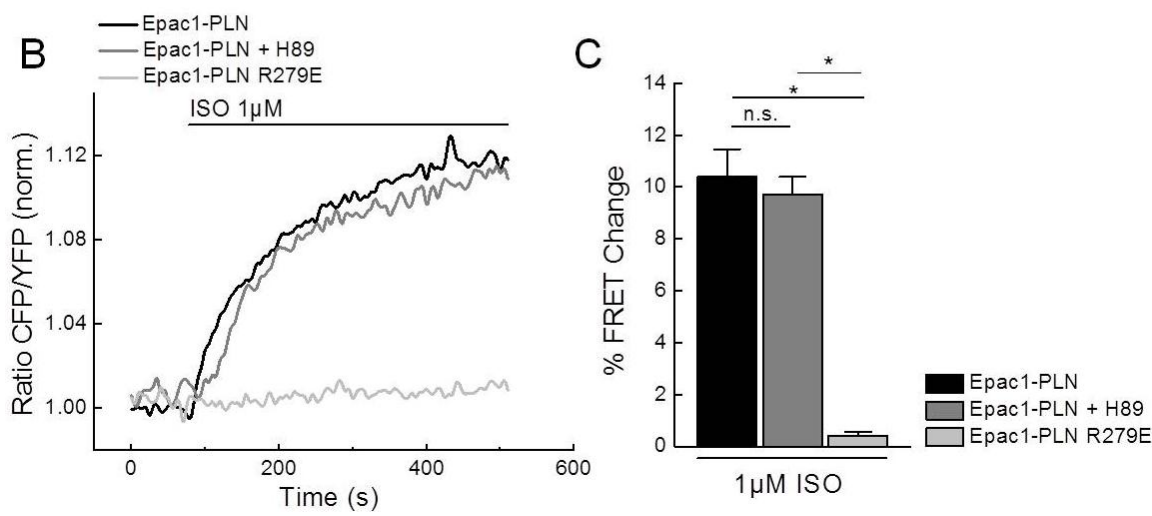

Figure 10. FRET responses of the Epac1-PLN under PKA inhibition and of the CAMP insensitive Epac1-PLN R279E mutant. (A) Cartoon of the Epac1-PLN and Epac1-PLN R279E FRET sensors. (B) Responses to CAMP stimulation with $1 \mu \mathrm{M}$ isoproterenol (ISO) in transiently transfected HEK293A cells show no significant differences in untreated and cells preincubated with the PKA inhibitor H-89 $(10 \mu \mathrm{M}$ for 10 minutes). Epac1-PLN R279E transfected HEK293A cells show no response to $1 \mu \mathrm{M}$ ISO.

Quantification is in (C), shown are means \pm SE, $n=8-12$ cells per group. * - significant difference at $p<0.05$.

After these control experiments in HEK293A, it was possible to start the generation of cardiomyocyte specific Epac1-PLN TG animals. Therefore, the full length pcDNA3.0 Epac1PLN sensor construct was subcloned into an $\alpha-\mathrm{MHC}$ expression vector via Xhol/ Kpnl double digestion (Figure $11 \mathrm{~A}$ ). Epac1-PLN positive clones showed a $\sim 2100$ bp fragment (representing the 2127 bp Epac1-PLN coding sequence) on a $1 \%$ agarose gel after control digestion (Figure $11 \mathrm{~B}$ ). This $\alpha-\mathrm{MHC}$ Epac1-PLN sensor construct was used for the generation of TG animals, expressing Epac1-PLN in cardiomyocytes. 

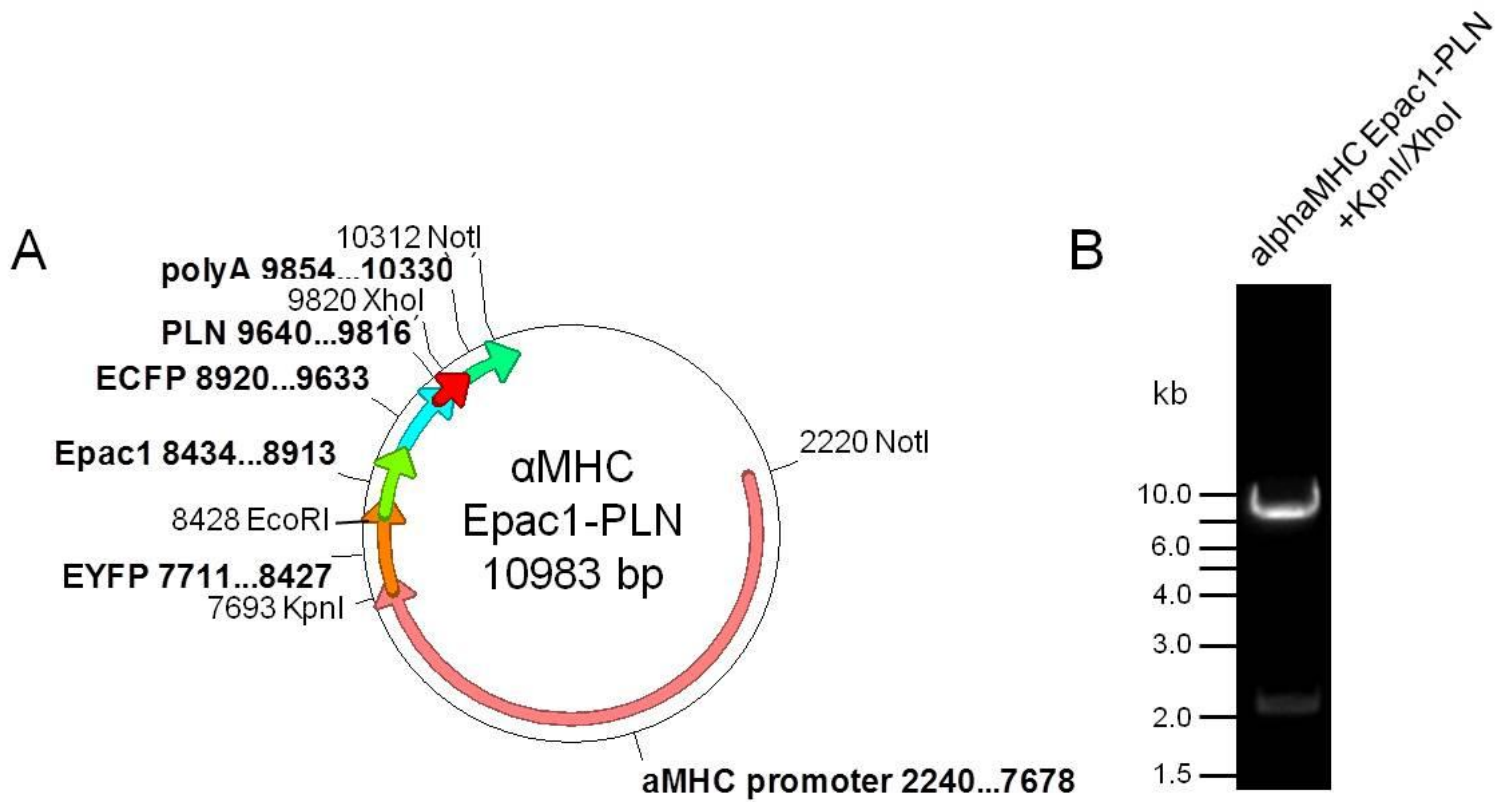

Figure 11. The $\alpha-M H C$ Epac1-PLN construct. (A) Plasmid map of the $\alpha-M H C$ Epac1-PLN construct. The $\alpha-M H C$ promoter allows cardiomyocyte specific expression of the FRET sensor. (B) Control digestion of the Epac1-PLN clones with $\mathrm{Kpnl} /$ Xhol leads to a 2100 bp DNA fragment (representing the 2127 bp Epac1-PLN coding sequence) on a 1\% agarose gel.

\subsection{Analysis of Epac1-PLN adenovirus}

The aim of this PhD thesis was to generate a TG mouse model expressing the Epac1-PLN FRET biosensor. In case of unexpected problems with TG mouse generation, it was important to have an alternative experimental system for the analysis of SERCA2a microdomain associated cAMP dynamics. Therefore, adenovirus expressing Epac1-PLN was
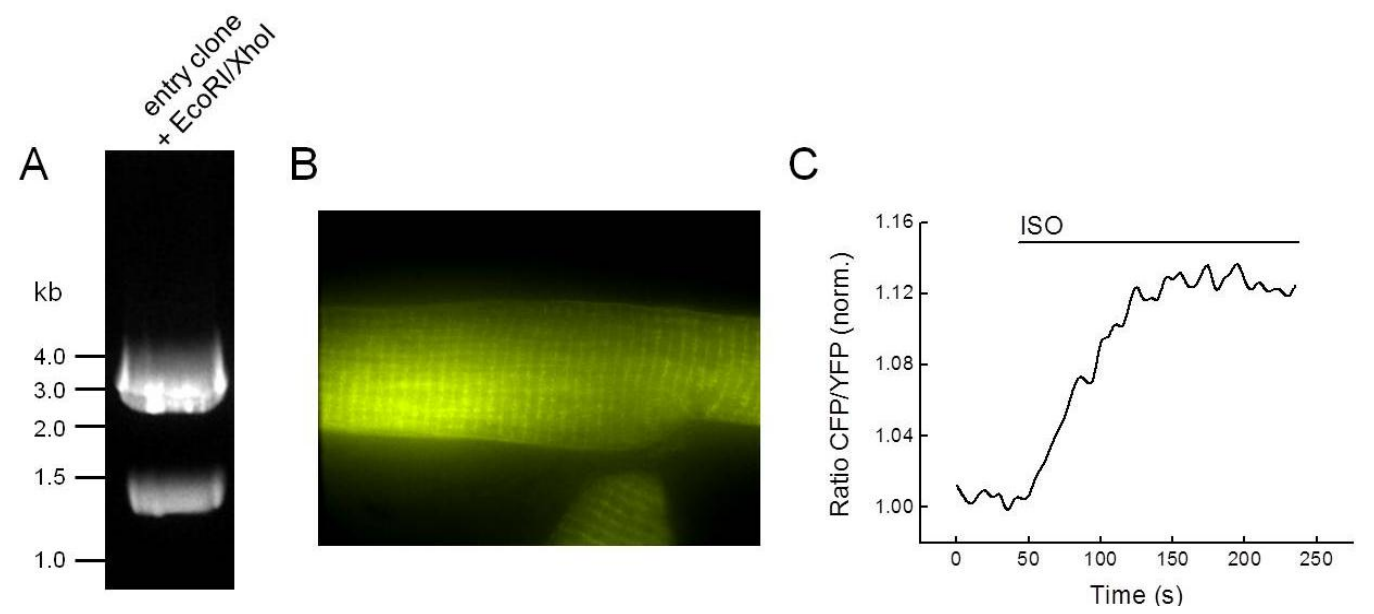

Figure 12. Epac1-PLN adenovirus. (A) Control digestion of the Epac1-PLN positive entry vector with EcoRI/ Xhol leads to a $1.4 \mathrm{kp}$ DNA fragment on a 1\% agarose gel. (B) Epifluorescent image of Epac1-PLN adenovirus transduced adult rat cardiomyocytes shows a striated sensor expression pattern. (C) Epac1-PLN adenovirus transduced adult rat cardiomyocytes respond to $\beta$-adrenergic stimulation with $100 \mathrm{nM}$ isoprenaline (ISO) with a change in FRET ratio, indicating the functionality of the Epac1-PLN sensor in these cells. 
generated using the Invitrogen Gateway ${ }^{\circledR}$ Cloning system. This adenovirus could be used for transduction of adult rat cardiomyocytes. When control digested with EcoRI/ Xhol, Epac1PLN positive entry clones showed a $1.4 \mathrm{~kb}$ fragment on a $1 \%$ agarose gel (Figure $12 \mathrm{~A}$ ). The positive entry clone was used for the generation of the adenoviral expression clone which was then transfected into HEK293A cells for Epac1-PLN adenovirus production. When analysed at the epifluorescence microscope, virus transduced adult rat cardiomyocytes showed an Epac1-PLN expression pattern that strongly resembled typical SERCA2a stainings (Figure $12 \mathrm{~B}$ ). Thus, the adenoviral Epac1-PLN sensor seemed to be localised in the desired destination within the cell. $\beta$-adrenergic stimulation of the transduced cardiomyocytes with ISO led to FRET changes similar to those in transfected HEK293A cells (Figure $9 \mathrm{~B}$ ), indicating that the adenoviral Epac1-PLN construct is functional in adult rat cardiomyocytes. However, because of the successful generation of the Epac1-PLN TG mouse line (see next section), the adenovirus approach was not further pursued within this PhD thesis.

\subsection{Generation and characterization of the transgenic Epac1-PLN mice}

To study cAMP dynamics under real time conditions in adult mouse ventricular cardiomyocytes, TG mice expressing the SERCA2a targeted Epac1-PLN sensor in adult myocardium (under the control of the $\alpha-M H C$ promoter, Figure 11) were generated. Pronuclear injections were performed at the Max Planck Institute for Experimental Medicine in Göttingen. The advantage of such a TG mouse model is that one can perform cAMP measurements in freshly isolated cardiomyocytes, excluding cultivation artefacts due to e.g. prolonged cell culture or adenoviral sensor transduction, thereby allowing the detection of cAMP dynamics under more physiological conditions.

\subsubsection{Generation of Epac1-PLN transgenic mice, sensor localisation and function}

Epac1-PLN TG mice were identified via standard genotyping PCR (Figure 13).

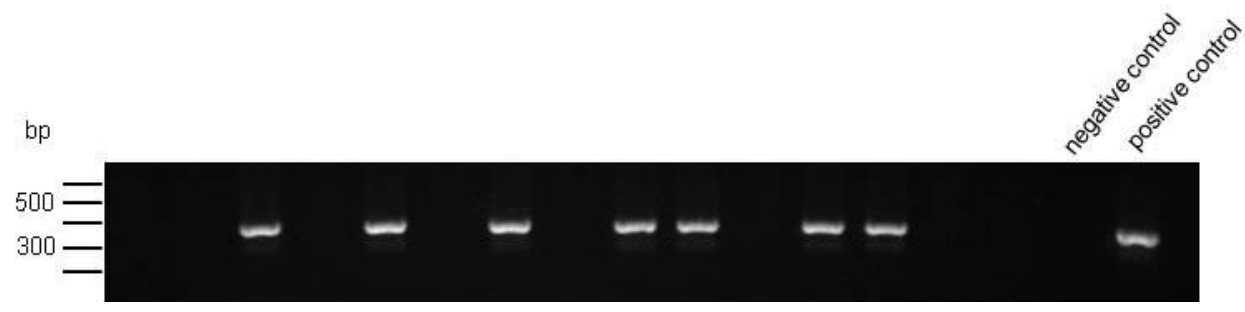

Figure 13. Representative Genotyping PCR. Positive bands were detected at $340 \mathrm{bp} . \mathrm{H}_{2} \mathrm{O}$ served as a negative, Epac1-PLN plasmid DNA as a positive control. $b p=$ basepairs 
Two positive founder mice were obtained one of which showed good breeding performance and sensor expression in virtually all cardiomyocytes of the offspring animals.

To confirm proper Epac1-PLN sensor localisation to the SERCA2a compartment, immunofluorescence analysis of TG heart cross sections of the offspring line were performed. Overlapping fluorescence signals for SERCA2a and Epac1-PLN were detected when analysed by confocal microscopy (Figure $14 \mathrm{~A}$ ). Besides, immunoblot analysis of heart fractions of TG and WT animals revealed Epac1-PLN localisation only in the membrane fraction of TG hearts together with SERCA2a, but not in the cytosol. The sensor was doubledetected via an anti-GFP antibody and an anti-PLN antibody against the fluorophores and the PLN part of the sensor construct, respectively, at $\sim 70 \mathrm{kDa}$ (Figure $14 \mathrm{~B}$ ). As mentioned in Figure 11, Epac1-PLN coding sequence is 2127 bp long, which should result in a $\sim 77 \mathrm{kDa}$ protein if the average amino acid size is supposed to be $110 \mathrm{Da}$. Therefore, it is assumed that the detected $\sim 70 \mathrm{kDa}$ band truly represents the Epac1-PLN sensor.

A
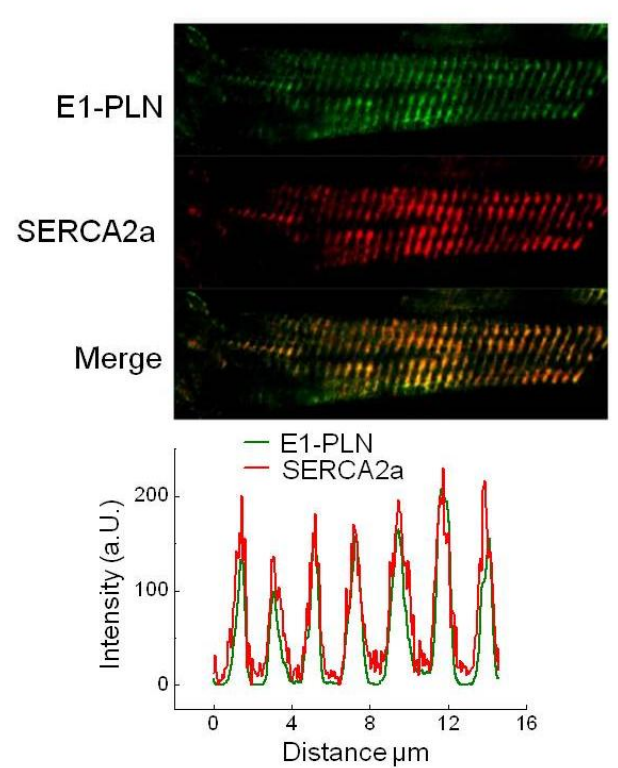

B

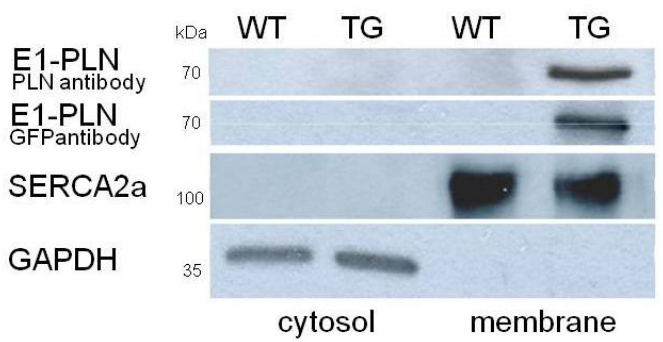

C
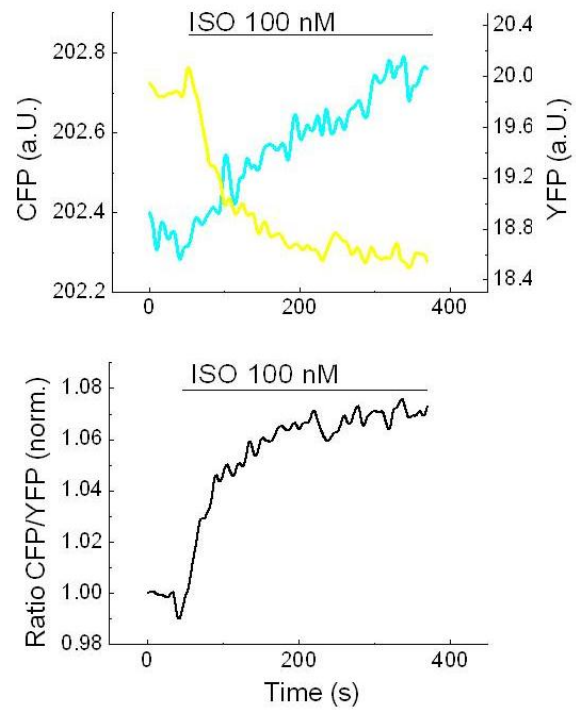
To test the function of the Epac1-PLN sensor in TG cardiomyocytes, freshly isolated cells were treated with the $\beta$-adrenergic agonist ISO. A clear increase of the CFP/YFP ratio, indicative of an increase in local microdomain cAMP levels, was detected and proved the sensor functionality in the TG animals (Figure $14 \mathrm{C}$ ).

\subsubsection{Characterization of Epac1-PLN transgenic mice}

TG and WT animals were compared in context of heart morphology, function and physiology to identify possible alterations in TG animals associated with Epac1-PLN expression.

WT and TG hearts showed no differences in heart-weight-to-body-weight (HW/BW) or heartweight-to-tibia-length (HW/TL) ratios at the age of 3 and 6 months (Figure $15 \mathrm{~A}$ ).

Furthermore, histological analysis revealed no alterations in cardiomyocyte dimensions of WGA-stained WT and TG heart cross section (Figure 15 B). Likewise, hematoxylin-eosin stainings also did not show any abnormalities in the TG Epac1-PLN hearts compared to

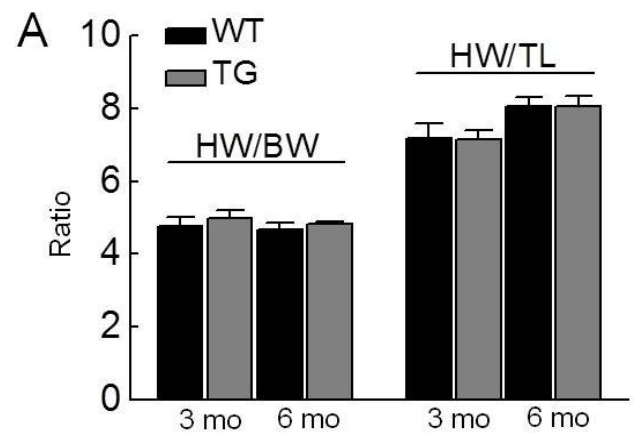

$\mathrm{B}$

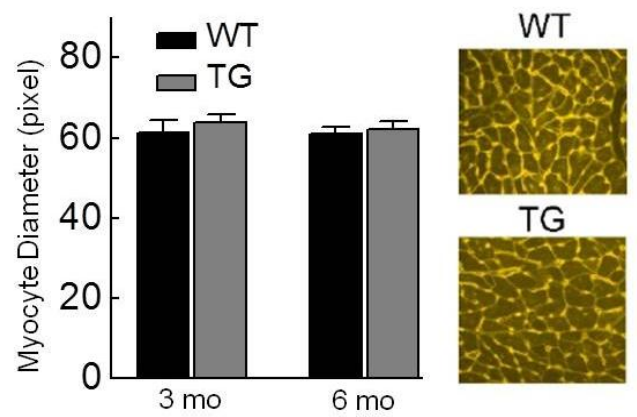

C

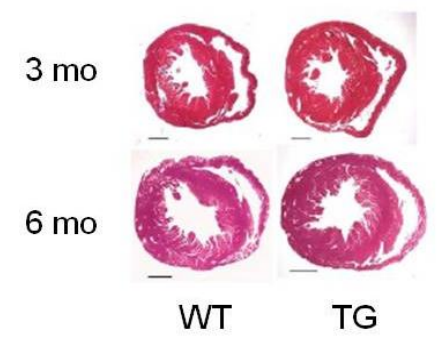

Figure 15. Morphometric characterization of Epac1-PLN transgenic mice. (A) Heart-weight-tobody-weight (HW/BW) and heart-weight-to-tibia-length (HW/TL) ratios at the age of 3 and 6 months do not show any abnormalities in the transgenic (TG) animals (means $\pm S E, n=10-11$ mice per group). (B) Diameter of TG Epac1-PLN cardiomyocytes was not altered compared to wildtype (WT) cells at the age of 3 and 6 months as investigated by WGA assay. Data are means \pm SE from 3-5 WT and TG hearts with 100 cells counted per each section. (C) Representative hematoxylin and eosin stainings of heart cross sections at the age of 3 and 6 months. Scale bars are $1 \mathrm{~mm}$.

those of WT littermates (Figure $15 \mathrm{C}$ ). 
Similar to endogenous PLN, Epac1-PLN (monomer $\sim 77 \mathrm{kDa}$ ) was also able to form oligomeric complexes as well as to exist in a monomeric form within TG cardiomyocytes (Figure 16 A). Besides, $\beta$-adrenergic stimulation of WT and TG cardiomyocytes induced a PKA dependent phosphorylation at Serine-16 $\left(\mathrm{P}^{\text {Ser16 }}\right)$ of the WT PLN and the TG sensor and endogenous PLN (Figure 16 B). PLN phosphorylation was analysed the pentamer and sensor oligomer level. Interestingly, WT cells showed a significant increase in PLN phosphorylation, whereas the differences in the TG sensor and endogenous PLN were not significantly altered compared to control conditions without ISO (Figure $16 \mathrm{C}$ ). However, when endogenous and sensor PLN of the TG cardiomyocytes were analysed as a single entity, the differences between ISO stimulated and control cells were also significant (Figure $16 \mathrm{D})$. This might indicate an interplay between endogenous and sensor PLN in the regulation of SERCA2a activity within TG cardiomyocytes after $\beta$-adrenergic stimulation. Taken together, these data strongly suggest that the Epac1-PLN sensor behaves like endogenous PLN and does not alter the physiology within the SERCA2a microdomain.

A

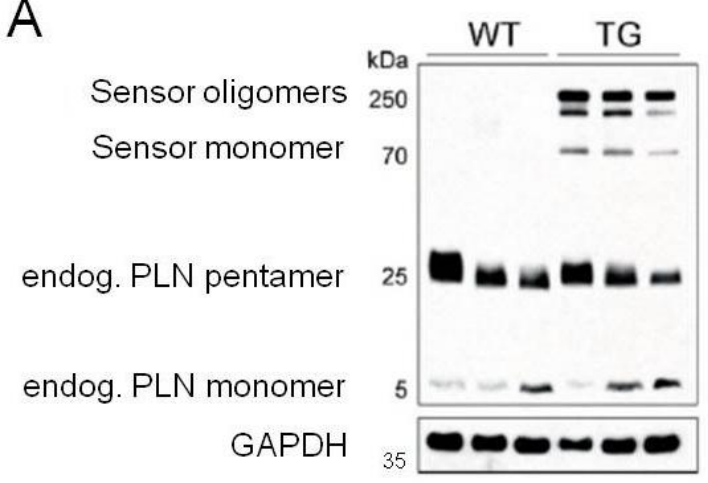

$B$

endog. PLN pentamer
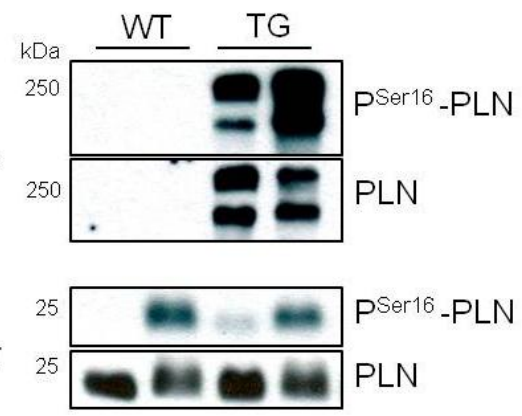
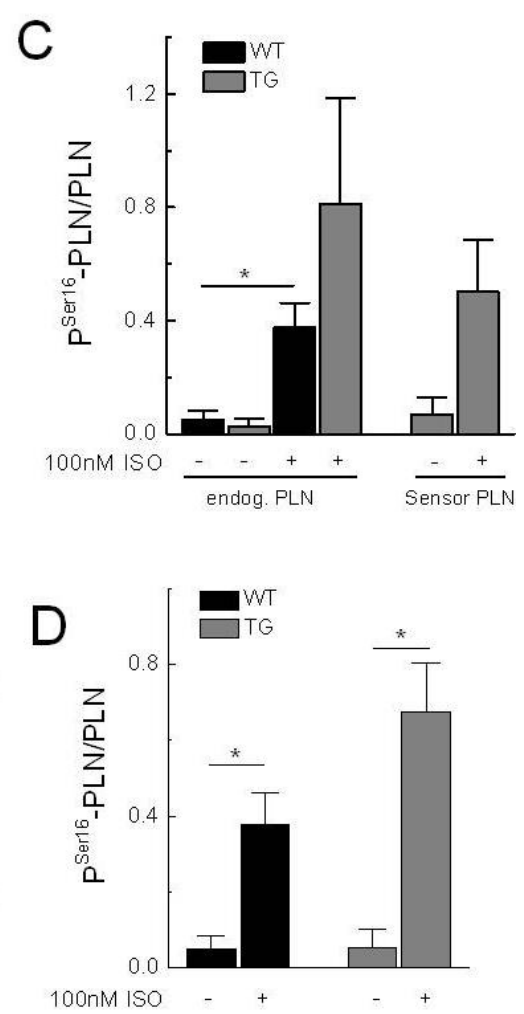

Figure 16. Transgenic Epac1-PLN construct can form oligomers and becomes PKA phosphorylated. (A) Similar to wildtype (WT) PLN, immunoblot analysis of transgenic (TG) cardiomyocytes shows the ability of Epac1-PLN (monomers at $\sim 77 \mathrm{kDa}$ ) to form oligomers. (B) Immunoblot analysis of WT and TG cardiomyocytes regarding PLN Serine-16 phosphorylation $\left(\mathrm{P}^{\text {Ser16 }}\right)$ confirms PKA-dependent phosphorylation of WT PLN pentamers and TG Epac1-PLN oligomers upon stimulation with $100 \mathrm{nM}$ of ISO. (C) Quantification of $P^{\text {Ser16 }}$ in WT PLN and TG endogenous and sensor PLN (D) Quantification of WT PLN and TG endogenous+sensor PLN. Means \pm SE, $n=3$ mouse hearts per group, ${ }^{*}$ - significant differences at $p<0.05$ 
Besides PLN phosphorylation after $\beta$-adrenergic stimulation, it was also important to exclude an abnormal phosphorylation of the Epac1-PLN sensor under basal conditions which might be associated with a modified regulation of the SERCA2a microdomain. Therefore, immunoblot analysis of WT vs. TG cardiomyocytes under non-stimulated conditions were performed and investigated regarding PKA ( $P^{\text {Ser16) }}$ (Figure $\left.17 \quad A, B\right)$ and CaMKII $\left(P^{\text {Thr17 }}\right)$ (Figure $17 \mathrm{C}, \mathrm{D})$ dependent PLN phosphorylation. No significant differences were detected in WT vs. TG total PLN phosphorylation patterns.
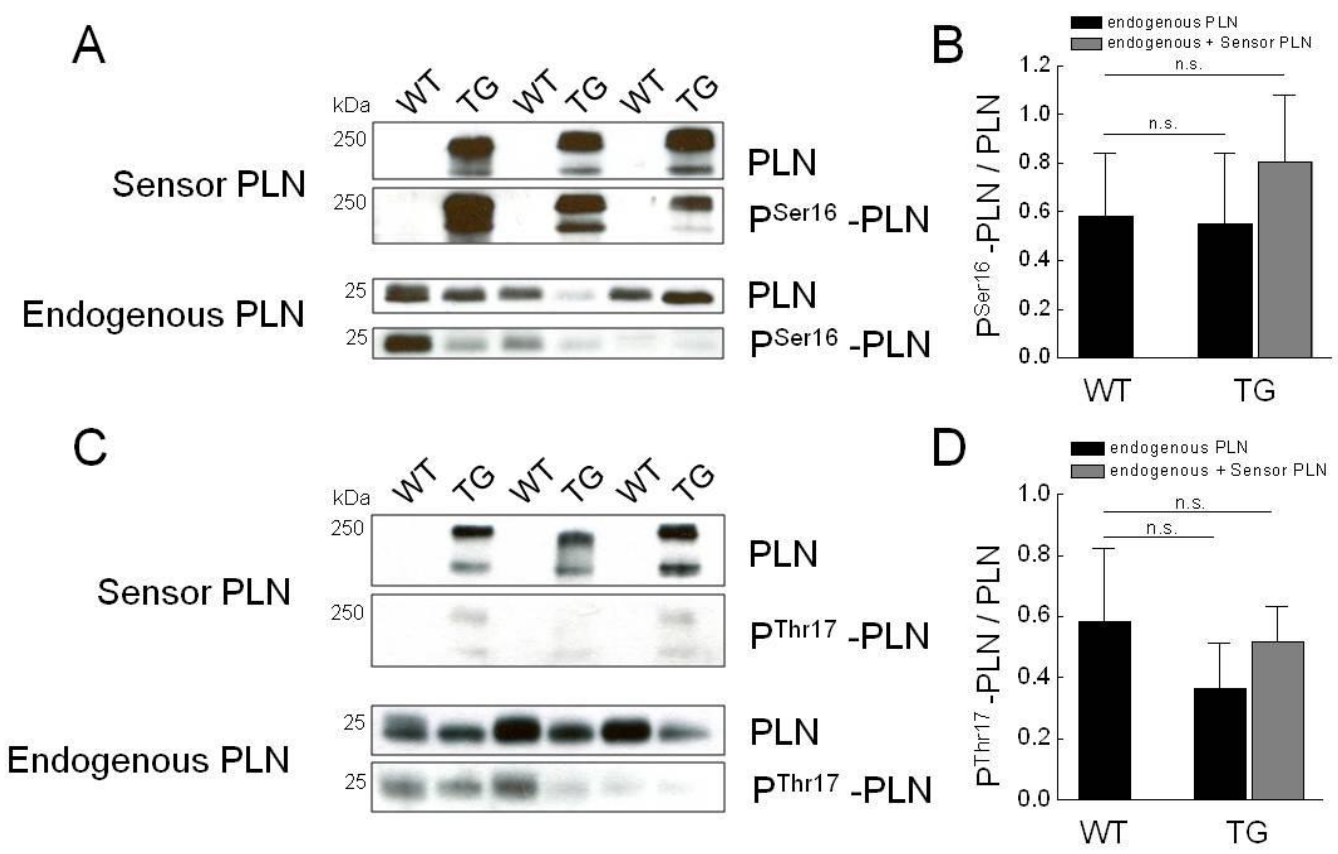

Figure 17. Basal PKA and CaMKII dependent PLN phosphorylation is not altered in transgenic (TG) vs. wildtype (WT) cardiomyocytes. (A,B) Immunoblot analysis of WT vs. TG Epac1-PLN cardiomyocytes revealed no significant differences in PKA dependent phosphorylation at Serine-16 $\left(P^{\text {Ser16 }}\right)$ in WT vs. TG cardiomyocytes. (C,D) Immunoblot analysis of WT vs. TG Epac1-PLN cardiomyocytes revealed also no significant differences in CaMKII dependent phosphorylation at Threonine-17 $\left(\mathrm{P}^{\mathrm{Thr} 17}\right)$ in WT vs. TG cardiomyocytes under basal conditions. Means $\pm \mathrm{SE}, n=3$ mouse hearts per group, ${ }^{*}$ - significant differences at $p<0.05$.

To analyse in vivo heart dimensions and function, echocardiography of WT and TG animals was performed. No dramatic alterations were revealed, apart from an increase in contractility in TG hearts (Table 11). 
Table 11. Echocardiographic phenotyping of the wildtype vs. Epac1-PLN transgenic mice at 3 months of age. Shown are means $\pm S E$, * - significant differences at $p<0.05$. LV-EDD, left ventricular enddiastolic dimension; LV-ESD, left ventricular end-systolic dimension; FS, fractional shortening; FAS, fractional area shortening; $E F$, ejection fraction; HR, heart rate; $n$, number of mice analysed per group.

\begin{tabular}{|l|l|l|}
\hline \multirow{2}{*}{ Parameter } & \multicolumn{2}{|l|}{ Genotype } \\
\cline { 2 - 3 } & Wildtype & Transgenic \\
\hline LV-EDD (mm) & $4.1 \pm 0.1$ & $3.9 \pm 0.1$ \\
LV-ESD (mm) & $2.7 \pm 0.1$ & $2.4 \pm 0.1^{*}$ \\
Septum (mm) & $0.82 \pm 0.02$ & $0.94 \pm 0.05^{*}$ \\
FS (\%) & $33.2 \pm 1.0$ & $38.3 \pm 0.9^{*}$ \\
FAS (\%) & $54.2 \pm 1.2$ & $61.8 \pm 1.3^{*}$ \\
EF (\%) & $60.0 \pm 1.1$ & $66.7 \pm 1.2^{*}$ \\
HR & $500 \pm 18$ & $462 \pm 16$ \\
$\mathrm{n}$ & 10 & 9 \\
\hline
\end{tabular}

Because Epac1-PLN sensor expression increases the natural amount of PLN in the TG cardiomyocytes, quantitative real-time PCR was performed to quantify PLN overexpression in TG Epac1-PLN hearts. It revealed a two-fold overexpression of PLN in Epac1-PLN hearts. Interestingly, SERCA2a expression in TG hearts analysed by immunoblot was also increased, ultimately leading to unchanged SERCA2a/PLN ratio (Figure 18 A-D).

Due to these alterations in protein expression, it was crucial to analyse calcium $\left(\mathrm{Ca}^{2+}\right)$ handling within the TG cardiomyocytes. $\mathrm{Ca}^{2+}$ decline kinetics $\left(\square_{\mathrm{Ca}}{ }^{2+}\right.$ ) in beating cardiomyocytes, as a measure of SERCA2a activity, showed no significant differences between WT and TG cardiomyocytes in absence and presence of ISO stimulation. Furthermore, SR $\mathrm{Ca}^{2+}$ load, determined by caffeine treatment of the cardiomyocytes, was unchanged (Figure $18 \mathrm{E}, \mathrm{F}$ ) in the TG vs. WT cells. These results indicate that $\mathrm{Ca}^{2+}$ handling in the TG Epac1-PLN cardiomyocytes is not altered compared to WT. 
A

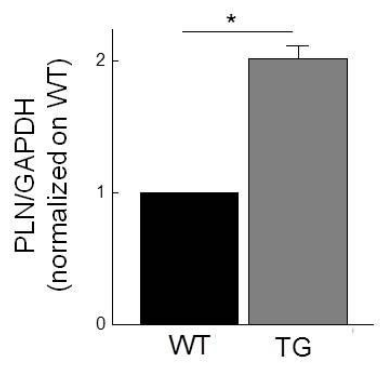

C

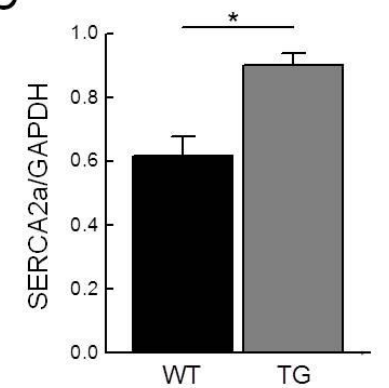

$E$

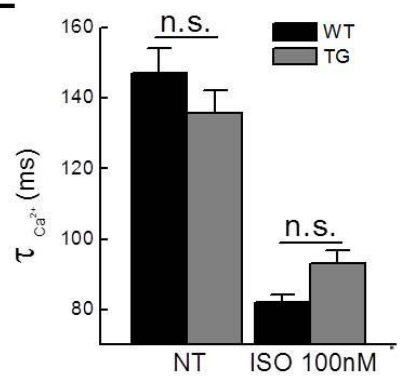

B

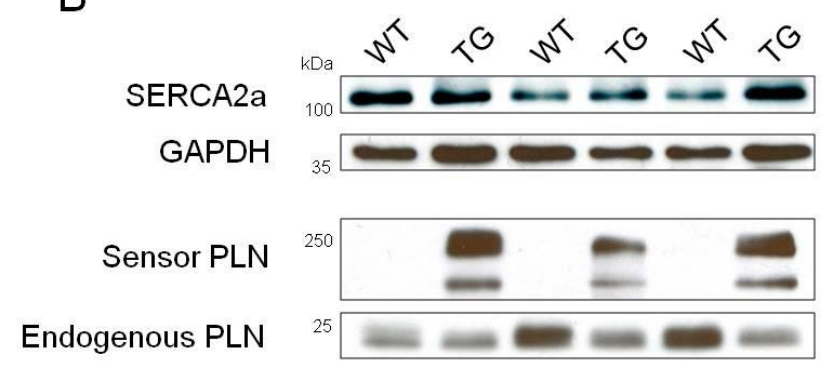

D

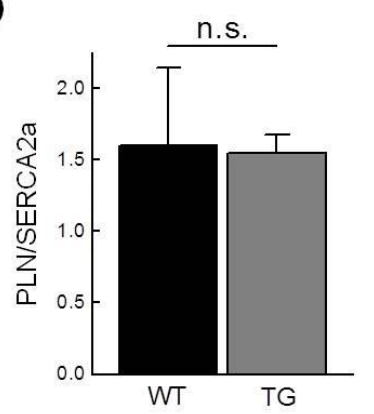

$\mathrm{F}$

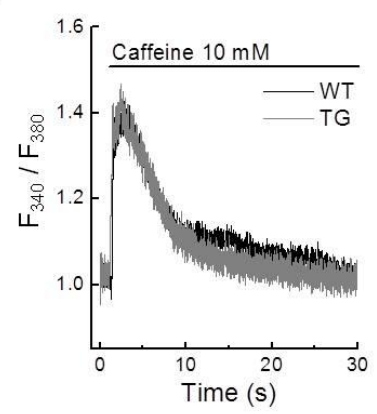

Figure 18. PLN and SERCA2a expression and calcium cycling. (A) Quantitative real time PCR analysis showed a $\sim 2$-fold overexpression of PLN in Epac1-PLN hearts (means \pm SE, $n=4$ mouse hearts per group). (B-D) Immunoblot analysis revealed an increase in SERCA2a protein expression but unchanged PLN/SERCA2a ratio (means \pm SE, $n=3$ mouse hearts per group, * - significant differences at $\mathrm{p}<0.05$ ) (E) Quantification of $\mathrm{Ca}^{2+}$ transients in beating cardiomyocytes loaded with Fluo-3 show no dramatic changes in $\mathrm{Ca}^{2+}$-decay time constant $\left(\tau_{\mathrm{Ca}}{ }^{2+}\right)$ in transgenic (TG) cardiomyocytes at basal and ISO (100 nM) stimulated states compared to wildtype (WT) (means \pm $\mathrm{SE}, \mathrm{n}=28-56$ cells from 2 hearts per group). (F) Analysis of sarcoplasmic reticulum (SR)-calcium load by the addition of caffeine to resting cardiomyocytes loaded with Fura-2 demonstrates no changes in TG compared to WT cells (means \pm SE, $n=18-19$ cells from 2 mouse hearts per group).

To further characterize the protein composition of Epac1-PLN expressing cardiomyocytes, immunoblot analysis of TG and WT cardiomyocyte lysates was performed. Besides SERCA2a (Figure $18 \mathrm{~B}$ ), further important $\mathrm{Ca}^{2+}$ handling proteins, such as the $\mathrm{NCX}$ and the LTCC (Cav1.2 as a subunit) were analysed. Surprisingly, NCX expression seemed to be slightly increased in the TG cardiomyocytes, whereas no significant alterations in Cav1.2 expression were detected (Figure 19). 
A

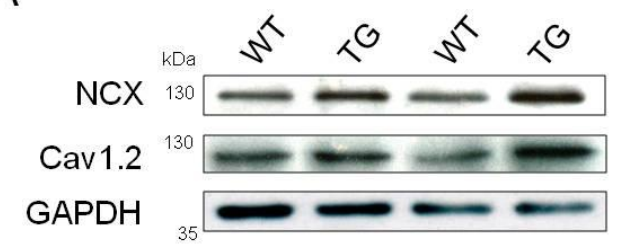

B

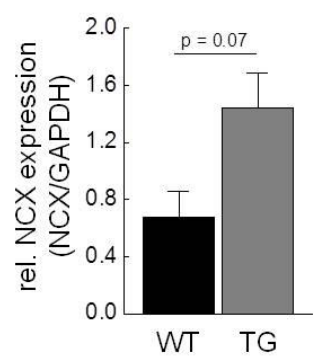

C

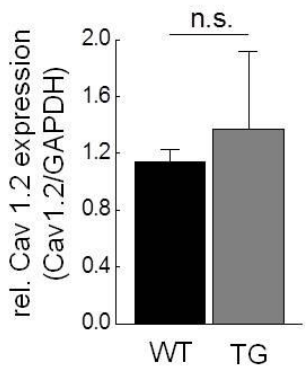

Figure 19. Immunoblot analysis of wildtype (WT) vs. Epac1-PLN transgenic (TG) cardiomyocytes regarding $\mathrm{NCX}$ and $\mathrm{Ca}_{\mathrm{v}} 1.2$ expression. (A-D) Immunoblot analysis of WT vs. TG Epac1-PLN cardiomyocytes revealed no significant differences in sodium calcium exchanger (NCX) or Ltype calcium channel ( $\left.\mathrm{Ca}_{\vee} 1.2\right)$ expression (means \pm SE, $n=3$ mouse hearts per group, * significant differences at $p<0.05$ ).

PDE4D is believed to play a crucial role for the regulation of cAMP dynamics within the SERCA2a microdomain and was not differentially expressed in TG vs. WT cardiomyocytes. Besides, no significant differences in the expression of PDE2 and PDE4D8, which has been shown to associate with the $\beta_{1}-A R$, were detected (Figure 20).

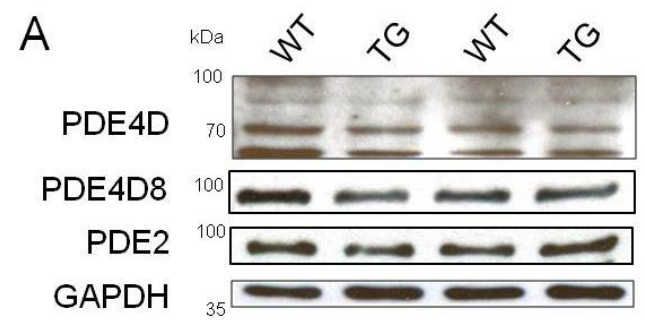

$\mathrm{B}$

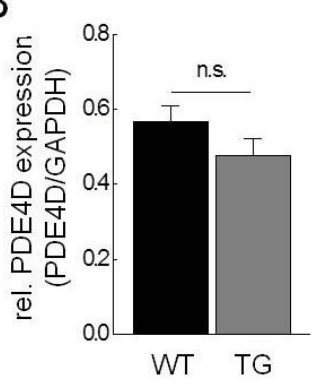

C

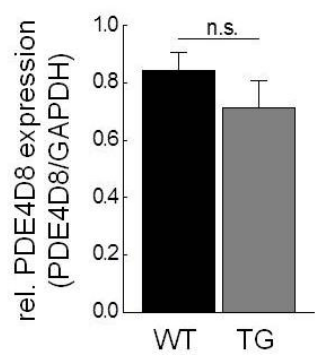

Figure 20. Immunoblot analysis of wildtype (WT) vs. Epac1-PLN transgenic cardiomyocytes regarding $P D E$ expression. (A-D) Immunoblot analysis of WT vs. TG Epac1PLN cardiomyocytes revealed no significant differences in total PDE4D, PDE4D8 and PDE2 expression (means \pm SE, $n=3-4$ mouse hearts per group, * - significant differences at $p<0.05$ ).

PKA is a crucial mediator between cAMP and its different downstream effectors. Therefore, it was important to analyse possible alterations in PKA-R subunit expression levels between WT and Epac1-PLN TG cardiomyocytes. This was also done via immunoblot analysis of TG vs. WT cardiomyocytes, and no difference in expression was detected (Figure 21). 
A

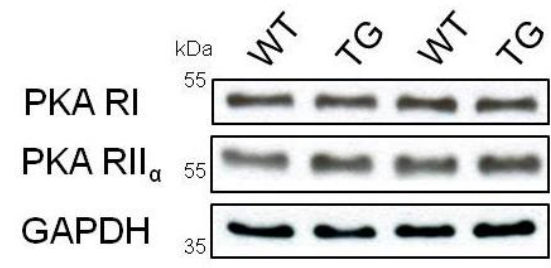

B

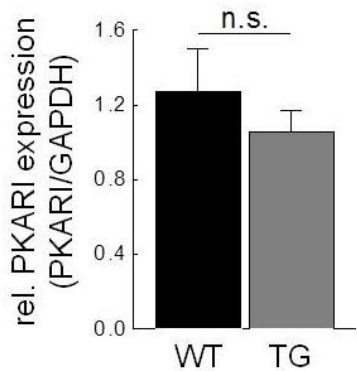

C

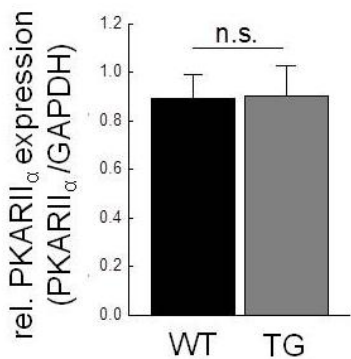

Figure 21. Immunoblot analysis of wildtype (WT) vs. Epac1-PLN transgenic (TG) cardiomyocytes regarding PKA expression. (A-C) Immunoblot analysis of WT vs. TG Epac1-PLN cardiomyocytes revealed no significant differences in PKA$\mathrm{RI}$ and PKA-RII ${ }_{\alpha}$ expression (means $\pm \mathrm{SE}, \mathrm{n}=4$ mouse hearts per group, * significant differences at

All these results indicate that the TG expression of the Epac1-PLN FRET biosensor does not dramatically affect cardiomyocyte function and protein expression patterns of the TG animals.

\subsection{FRET measurements in Epac1-camps vs. Epac1-PLN cardiomyocytes}

To investigate the molecular mechanisms that confine the SERCA2a microdomain from the bulk cytosol, cAMP FRET measurements in freshly isolated adult cardiomyocytes from previously established Epac1-camps (Calebiro et al., 2009) (cytosolic cAMP FRET sensor) and Epac1-PLN (SERCA2a targeted cAMP FRET sensor) TG mice were performed. Differences in cAMP dynamics should give a deeper insight into the regulation and maintenance of subcellular cAMP microdomains.

\subsubsection{FRET measurements in Epac1-camps and Epac1-PLN cardiomyocytes reveal} PDE dependent $\beta$-adrenergic receptor ( $\beta$-AR) signaling

In the first series of experiments, FRET recordings under selective stimulations of $\beta_{1}$ - and $\beta_{2^{-}}$ ARs were conducted to analyse contributions of individual $\beta-A R$ subtypes into cAMP signals. Interestingly, strong $\beta_{1}$-AR-cAMP signals were present in both the SERCA2a microdomain and the bulk cytosol (Figure $22 \mathrm{~A}, \mathrm{~B}$ ), while much smaller $\beta_{2}$-AR-cAMP responses were detectable only in the cytosol and barely "reached" the SERCA2a microdomain (Figure 22 
C,D). Unexpectedly, the relative amplitudes of $100 \mathrm{nM}$ isoproterenol (ISO) induced $\beta$ adrenergic FRET change and selective $\beta_{1}$-AR responses measured with Epac1-PLN were higher than the signals recorded in the bulk cytosol via Epac1-camps (Figure $22 \mathrm{E}$ ). This difference was still present when using even more saturating ISO concentrations of $300 \mathrm{nM}$ (Figure $22 \mathrm{~F}$ ).
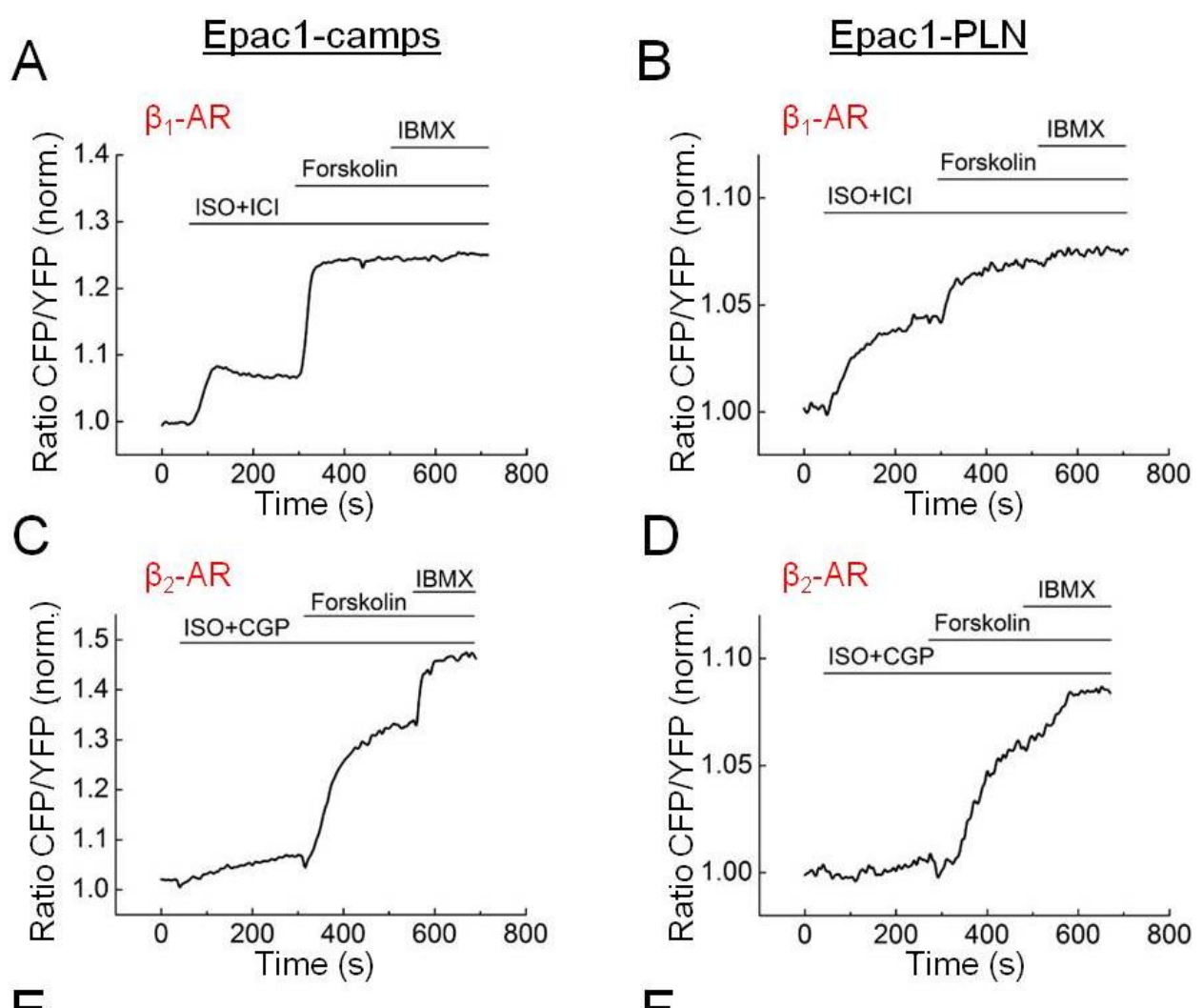

$E$

$\mathrm{F}$
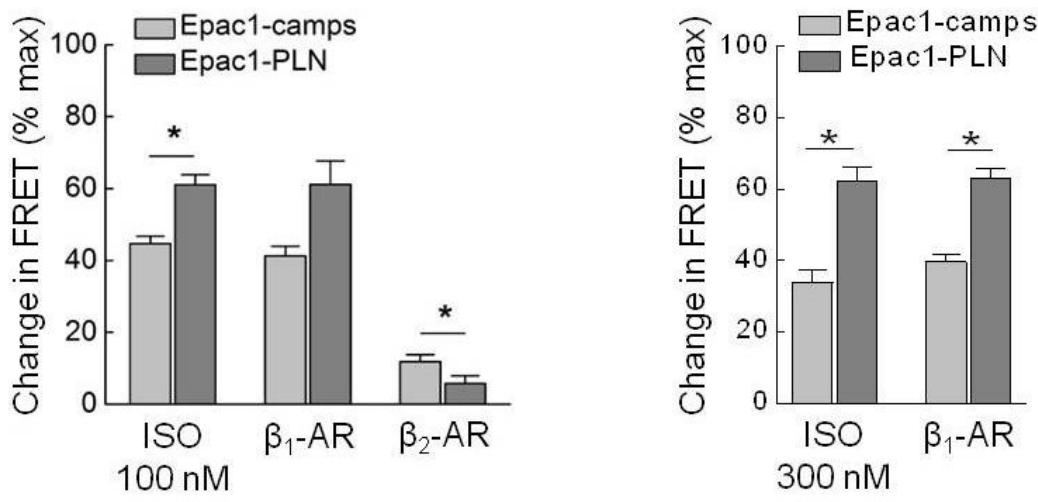

Figure 22. $\beta_{1}$-AR stimulation induces stronger FRET changes in the SERCA2a compartment compared to the bulk cytosol. (A,B) Representative FRET traces from Epac1-camps and Epac1PLN cardiomyocytes upon $\beta_{1}$-AR-selectively stimulation (100 nM ISO plus $50 \mathrm{nM}$ of the $\beta_{2}$-AR blocker ICI 118551). (C,D) Representative FRET traces from Epac1-camps and Epac1-PLN myocytes after $\beta_{2}$-AR-selective stimulation (100 nM ISO plus $100 \mathrm{nM}$ of the $\beta 1$-AR blocker CGP-20712A). $\beta_{2}$-AR had almost no effect on CAMP in the SERCA2a compartment. Maximal stimulation of adenylyl cyclase was achieved by $10 \mu \mathrm{M}$ of forskolin and the maximal FRET response was induced by the unselective PDE inhibitor IBMX $(100 \mu \mathrm{M})$. (E) Quantification of the FRET experiments shown in A-D. $\beta-A R$ and selective $\beta_{1}$-AR stimulations led to stronger FRET responses in the SERCA2a microdomain compared to bulk cytosol. (F) Quantification of FRET experiments in Epac1-camps and Epac1-PLN cardiomyocytes after $\beta$-adrenergic stimulation with $300 \mathrm{nM}$ ISO. Means $\pm \mathrm{SE}, \mathrm{n}=8-10$ cells from 3 mouse hearts per condition. 
The FRET experiments were performed in resting cardiomyocytes devoid of electrical field stimulation to avoid signal artefacts due to movement of the cell and to increase cell survival during the measurements. However, in vivo, mouse hearts show a contraction rate of 500600 bpm including a constant and rapid $\mathrm{Ca}^{2+}$ cycling within each cardiomyocyte during each contraction cycle. To test if these rapid changes in $\mathrm{Ca}^{2+}$ concentration in the SERCA2a microdomain under electric field stimulation might lead to different FRET results than in resting cells, $\beta$-adrenergic responses in resting and paced Epac1-PLN cardiomyocytes were analysed. However, there were no significant differences detected (Figure 23), justifying the use of resting cardiomyocytes in the following FRET experiments.

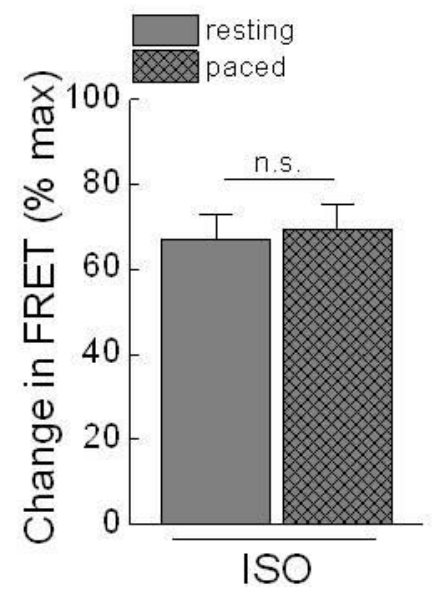

\begin{abstract}
Figure 23. $\beta$-AR stimulation in Epac1-PLN cardiomyocytes under resting and field stimulated conditions. Quantification of FRET experiments in Epac1-PLN cardiomyocytes after $\beta$-adrenergic stimulation with $100 \mathrm{nM}$ ISO under resting and electric field stimulated conditions (paced at $1 \mathrm{~Hz}, 20-30 \mathrm{~V}$ ). No significant differences between the two groups were detected. Means $\pm S E, n=6$ cells per condition from the same mouse heart.
\end{abstract}

To rule out the possibility that differences in cytosolic and SERCA2a microdomain specific FRET signals are due to different sensor affinities, sensitivities of both sensors to cAMP in presence of the adenylyl cyclase inhibitor MDL12,330A were directly compared and found a slightly lower affinity of the targeted Epac1-PLN $\left(E_{50}=5.3 \pm 1.1 \mu \mathrm{M}\right)$ sensor compared to the cytosolic Epac1-camps $\left(\mathrm{EC}_{50}=1.8 \pm 0.3 \mu \mathrm{M}\right)$ (Figure $\left.24 \mathrm{~A}\right)$. Besides, stimulation of Epac1-camps or Epac1-PLN cells with increasing ISO concentrations revealed enhanced sensitivity of Epac1-PLN to ISO (Figure $24 \mathrm{~B}$ ). These results might imply that the differences between the $\beta-A R$ responses in the SERCA2a microdomain and the bulk cytosol are even bigger than detected. 
A

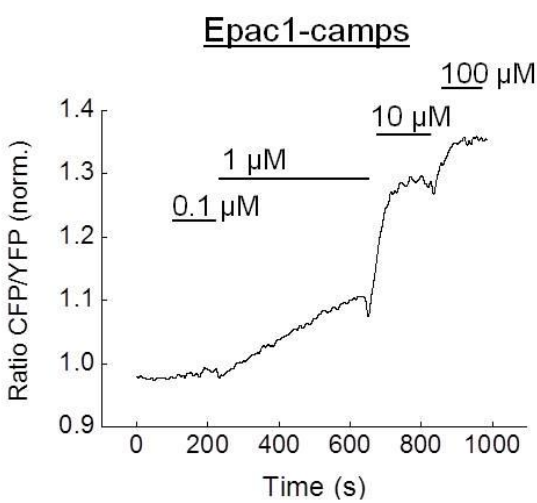

C

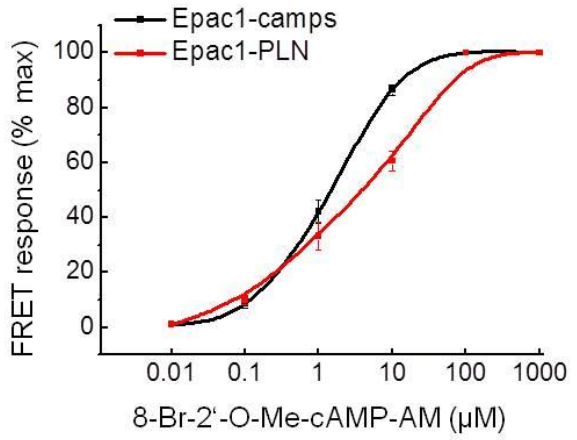

$\mathrm{B}$

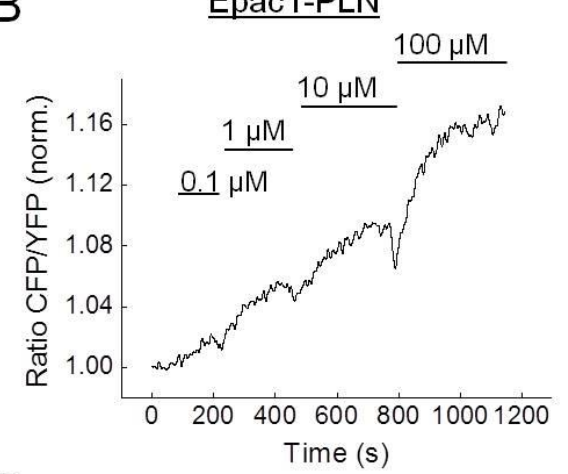

D

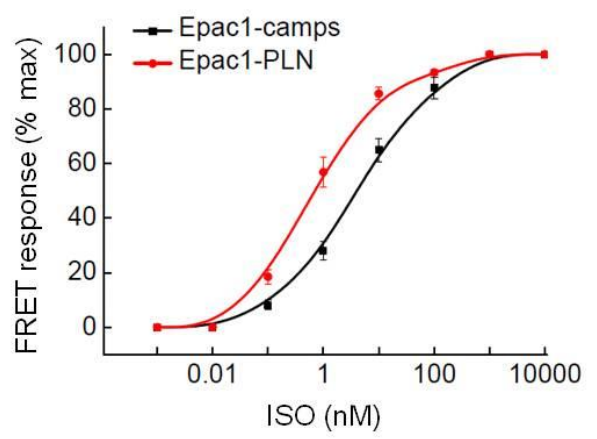

Figure 24. Sensitivity of Epac1-camps and Epac1-PLN to cAMP and increasing isoproterenol (ISO) concentrations. (A,B) Representative FRET traces In Epac1-camps and Epac1-PLN cardiomyocytes treated with increasing concentrations of the membrane-permeable cAMP analogue 8-Br-2"-O-Me-cAMP-AM (having the same affinity for Epac1 as cAMP but being resistant to degradation via PDEs). (C) Concentration-response dependencies for 8-Br-2"-O-Me-cAMP-AM measured with Epac1-camps $\left(\mathrm{EC}_{50}=1.8 \pm 0.3 \mu \mathrm{M}\right)$ and Epac1-PLN $\left(\mathrm{EC}_{50}=5.3 \pm 1.1 \mu \mathrm{M}\right)$ transgenic cardiomyocytes ( $n=8-11$ cells from 2-3 mice each). Measurements were performed in the presence of the adenylyl cyclase inhibitor MDL12,330A (100 $\mu \mathrm{M})$. (D) Stimulation of Epac1-camps or Epac1-PLN cells with increasing ISO concentrations reveals enhanced sensitivity of Epac1-PLN to ISO ( $\mathrm{n}=6$ cells from 2-3 mouse hearts).

Using these in vitro calibration curves (Figure 24) and a previously described protocol (Borner et al., 2011), FRET ratios from Figure 22 could be converted into absolute cAMP concentrations. ISO stimulated CAMP values in the SERCA2a microdomain were about 4fold higher as compared to the bulk cytosol $(3.9 \pm 0.4 \mu \mathrm{M}$ in the cytosol vs. $15.3 \pm 2.8 \mu \mathrm{M}$ at SERCA2a, respectively, means $\pm \mathrm{SE}, \mathrm{p}<0.01)$.

To understand the mechanism behind this cAMP channelling between the $\beta-A R$ and the SERCA2a microdomain, TG cardiomyocytes were pre-treated with different PDE inhibitors and then stimulated with a sub-maximal dose of ISO (Figure 25). Pre-inhibition of PDE3 or PDE4 alone did not compensate ISO induced differences in FRET change between the cytosol (Epac1-camps sensor) and the SERCA2a microdomain (Epac1-PLN sensor) (Figure 25 A,B). Interestingly, treatment with combined PDE3 and PDE4 inhibition abolished the differences in $\beta$-adrenergic responses (Figure $25 \mathrm{C}$ ). Besides, unselective PDE inhibition with 3-isobutyl-1-methylxanthin (IBMX) also blunted the difference in ISO induced FRET responses between the cytosol and the SERCA2a microdomain (Figure 25 D,E). These 
results suggest that two PDEs act in concert to facilitate the direct receptor-microdomain communication between membrane-localised $\beta_{1}-A R$ and the SERCA2a-associated microdomain. These data also show that the targeted sensor and the new mouse model can be used to monitor the local, microdomain-specific pool of cAMP which is different from the cAMP levels measured in the bulk cytosol.
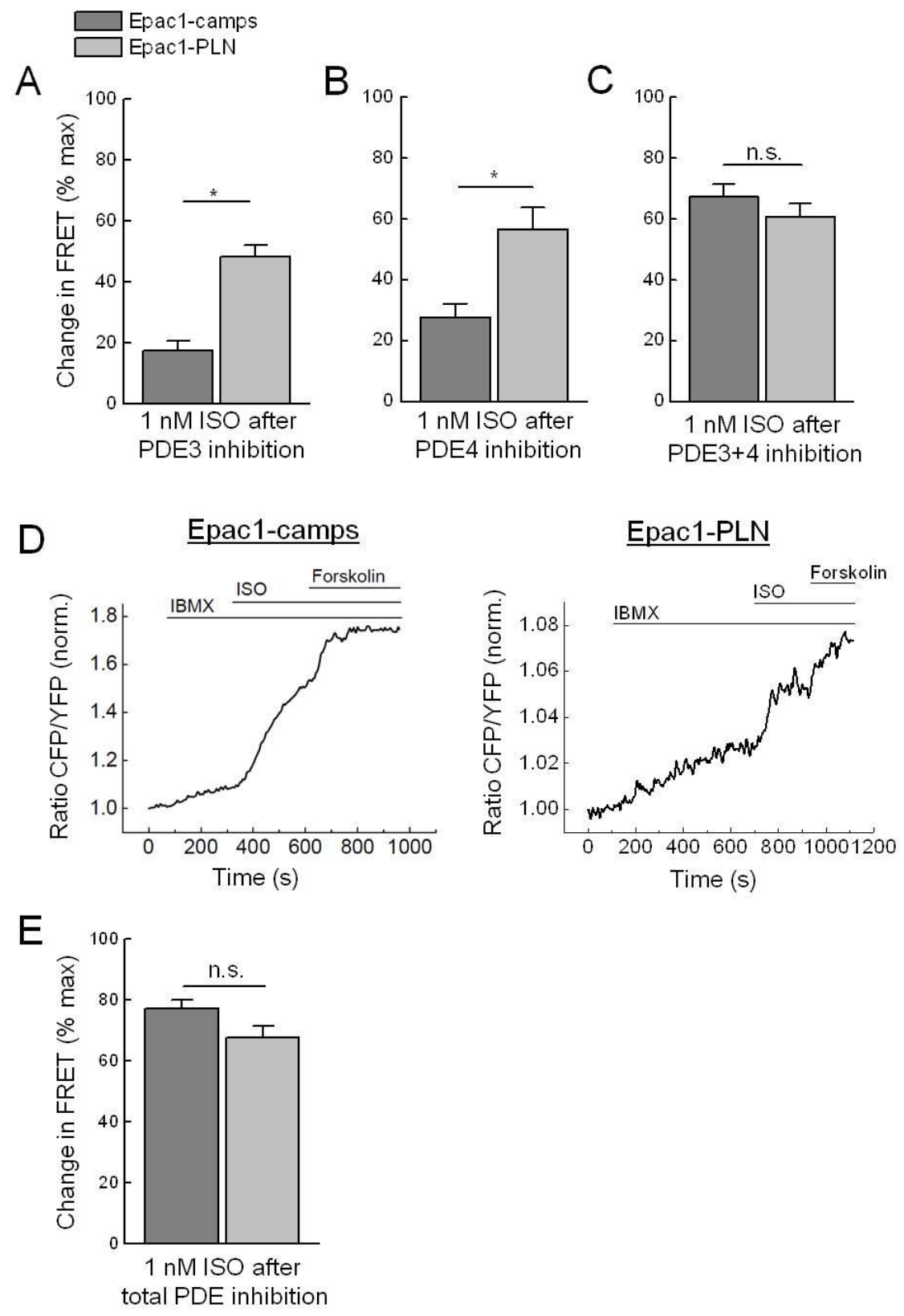

Figure 25. The difference between Epac1-camps and Epac1-PLN in the magnitude of $\beta$-ARcAMP signals is abolished after PDE pre-inhibition. (A,B,C) Quantification of FRET experiments in Epac1-camps (cytosolic sensor) and Epac1-PLN (SERCA2a specific sensor)

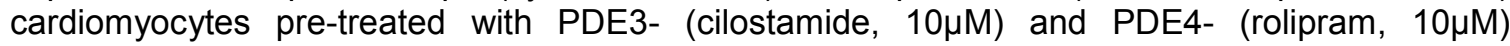
inhibitors and subsequently with a sub-maximal dose of isoproterenol (ISO, $1 \mathrm{nM}$ ) for $\beta$-AR stimulation. (D) Representative FRET traces from Epac1-camps and Epac1-PLN transgenic cardiomyocytes pre-treated with the unselective PDE inhibitor IBMX $(100 \mu \mathrm{M})$. Forskolin $(10 \mu \mathrm{M})$ was used to achieve complete adenylyl cyclase activation at the end of each experiment. (E) Quantification of FRET experiments shown in D. Means \pm SE, $n=10-16$ cells from 3-4 mouse hearts per condition. ${ }^{*}$ - significant differences at $p<0.05$. 


\subsubsection{PDE profiles after $\beta$-adrenergic stimulation in Epac1-camps and Epac1-PLN cardiomyocytes analysed by FRET}

PDEs are most important for shaping cAMP dynamics within cardiomyocytes, especially after $\beta$-adrenergic stimulation. To investigate which PDE family plays the predominant role in the control of $\beta$-AR cAMP signal termination within the cytosol vs. the SERCA2a compartment, freshly isolated cardiomyocytes from Epac1-camps (cytosolic sensor) and Epac1-PLN (SERCA2a targeted sensor) mice were treated with ISO and subsequently with several PDE specific inhibitors (Figure 26 A,B). As appropriate PDE1 and PDE8 specific inhibitors are still lacking and PDE2-4 represent the predominant PDE families in the heart, the following experiments focussed on PDE2, PDE3 and PDE4 inhibition.
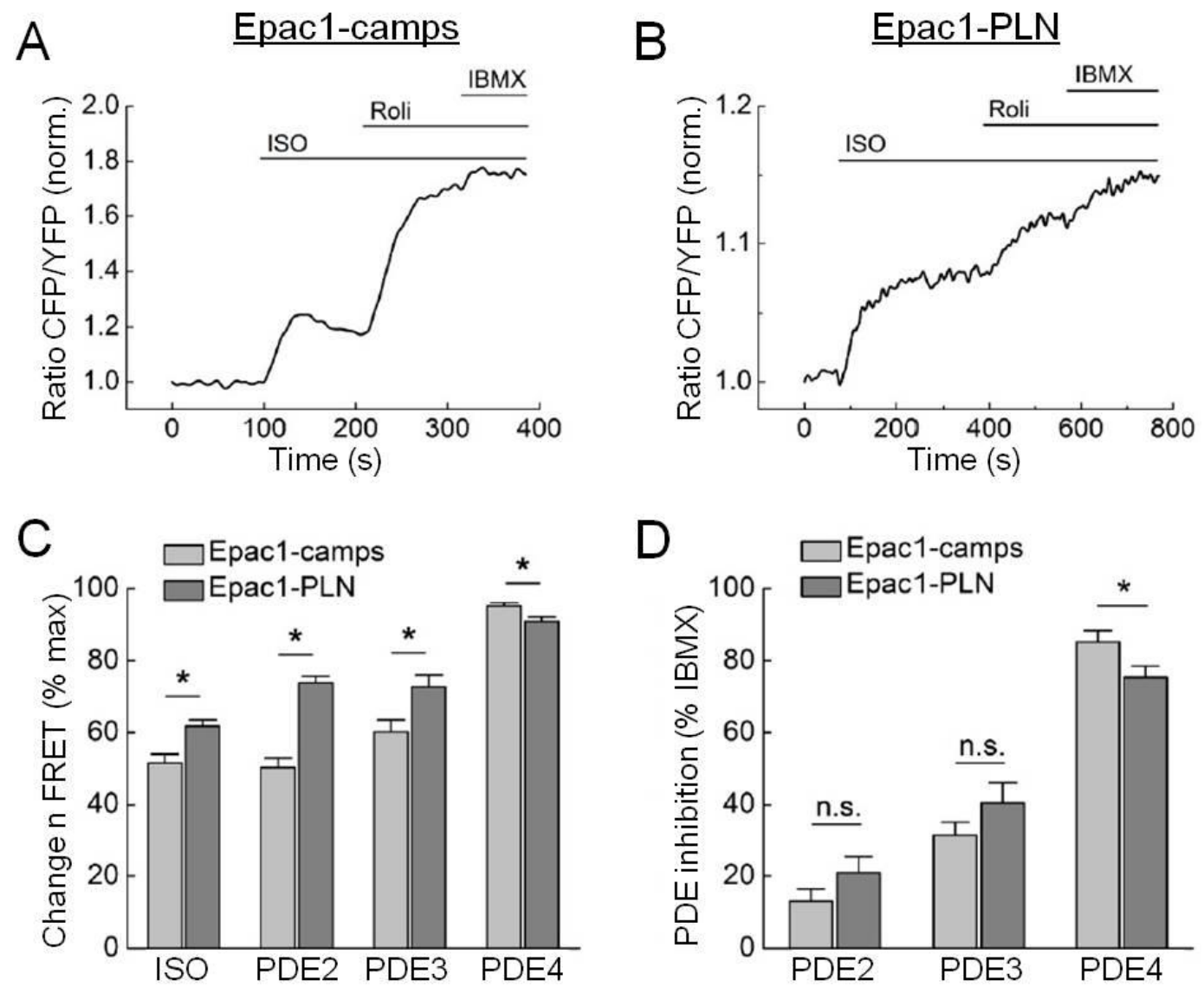

Figure 26. PDE4 plays the predominant role for cAMP degradation after $\beta$-AR stimulation in both the cytosol and the SERCA2a compartment. (A,B) Representative FRET traces of Epac1camps and Epac1-PLN cardiomyocytes after $\beta$-AR stimulation with isoprenaline (ISO) and subsequent PDE4 inhibition with rolipram (Roli). IBMX is used to induce total PDE inhibition. (C) Quantification of PDE inhibitor experiments after $\beta$-AR stimulation revealed significantly higher response to ISO in the SERCA2a compartment compared to the bulk cytosol leading to significantly higher ISO-PDE inhibitor percentage in the SERCA2a compartment. To circumvent this fact, PDE inhibition was quantified after ISO stimulation (D) showing a significantly higher PDE4 contribution to cAMP hydrolysis in the bulk cytosol compared to the SERCA2a compartment. However, PDE4 also plays the predominant role in the SERCA2a microdomain. Means \pm SE, $n=10-12$ cells from 3 mouse hearts per condition. * - significant differences at p <0.05. Substance concentrations: ISO 100 nM, IBMX $100 \mu \mathrm{M}$, BAY 60-7550 100 nM (PDE2 inhibition), cilostamide $10 \mu \mathrm{M}$ (PDE3 inhibition), rolipram $10 \mu \mathrm{M}$ (PDE4 inhibition). 
ISO stimulation led to a significantly higher cAMP-FRET response in the SERCA2a microdomain compared to the bulk cytosol (Figure $26 \mathrm{C}$, Figure 22). To avoid imprecise quantification of PDE contributions (Figure $26 \mathrm{C}$ ), only the percentage of individual PDE inhibitor effects, without the ISO portion, from the total PDE inhibition by the unselective inhibitor IBMX was quantified (Figure $26 \mathrm{D}$ ). In this case, the contribution of PDE4 after $\beta$ adrenergic stimulation was significantly higher in the cytosol vs. the SERCA2a compartment.

\subsubsection{Basal PDE profiles in Epac1-camps and Epac1-PLN cardiomyocytes analysed by FRET}

PDEs have been shown to shape subcellular cAMP gradients after $\beta$-AR stimulation and to segregate individual subcellular cAMP microdomains from the bulk cytosol. In the next step, FRET analysis of PDE contributions under basal conditions were performed, to identify PDE families crucial for confining the SERCA2a compartment in absence of $\beta$-adrenergic stimulation and for regulating the access of cytosolic cAMP pools to the microdomain. Therefore, TG cardiomyocytes were treated with several specific PDE inhibitors without ISO prestimulation. Almost no changes in the FRET ratio of Epac1-camps expressing cardiomyocytes upon inhibition of any PDE family was detected, whereas a significantly higher contribution to CAMP degradation by PDE3 and especially PDE4 was detected in the SERCA2a compartment compared to the cytosol (Figure 27 A-E) . Immunoblot analysis of freshly isolated WT cardiomyocytes showed that these PDE inhibitors could increase PLN phosphorylation when applied without ISO pre-stimulation (Figure $27 \mathrm{~F}$ ). These results confirmed that the FRET experiments correlated well with the PKA dependent phosphorylation of PLN and that PDE3 and 4 are involved in functional segregation of the SERCA2a microdomain from the bulk cytosol. 

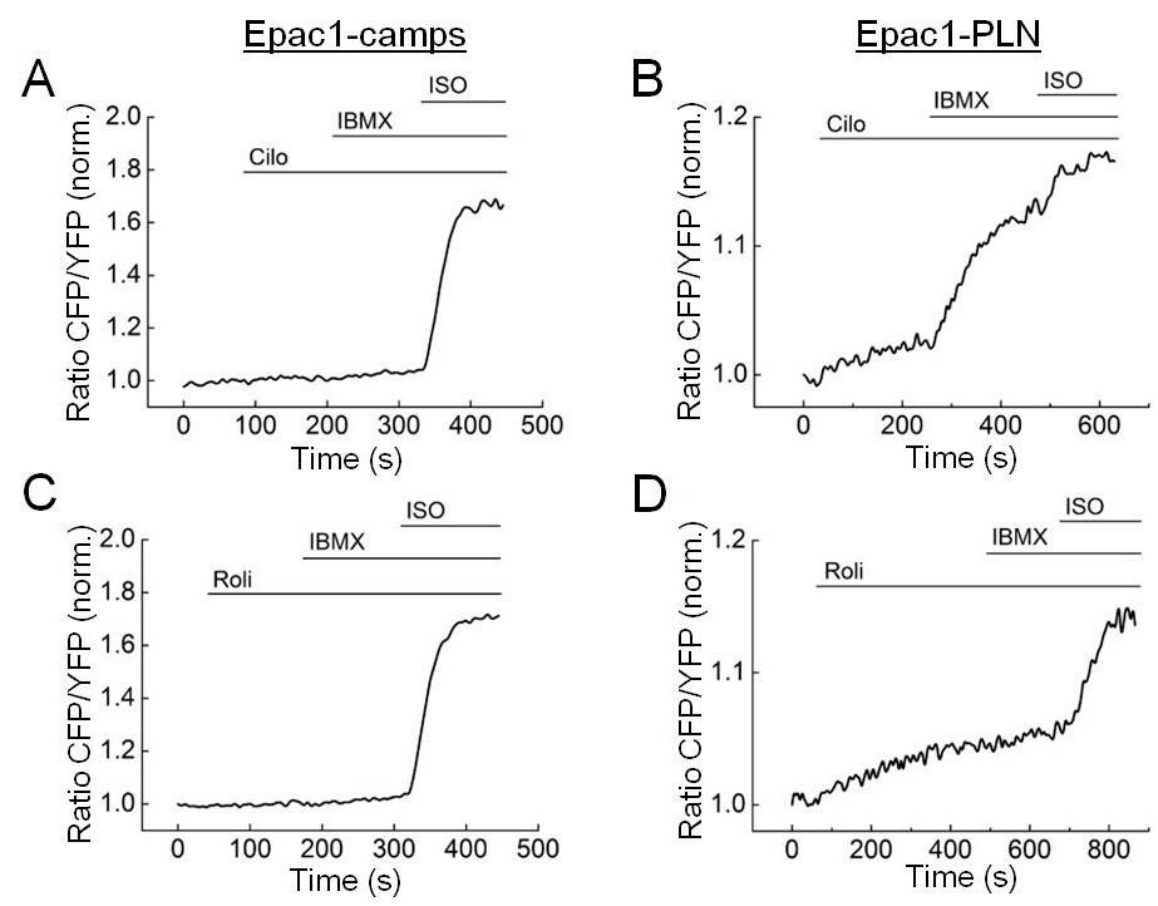

E
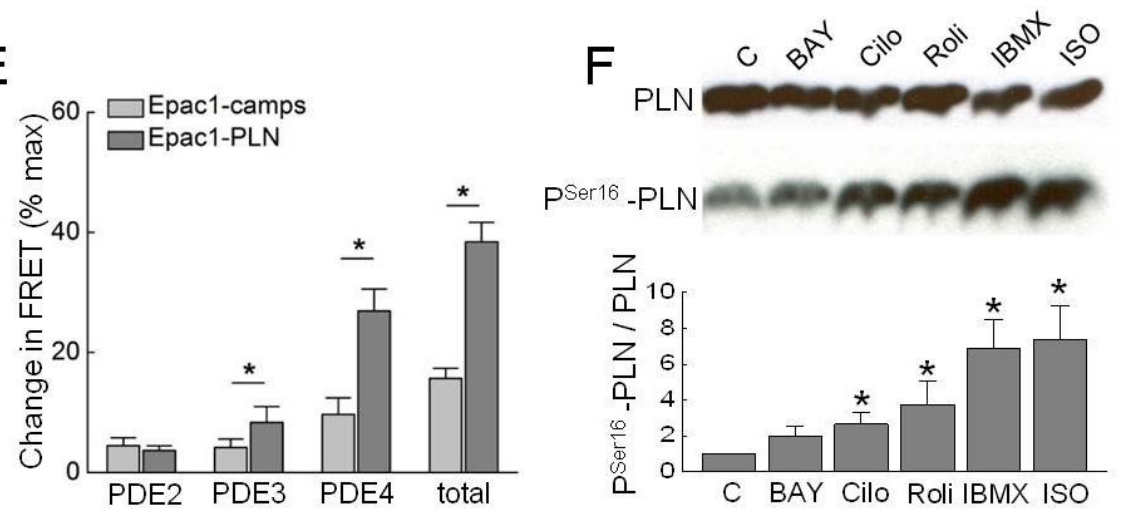

Figure 27. PDE contribution to CAMP hydrolysis under basal conditions. (A,B) Representative FRET traces of Epac1-camps and Epac1-PLN transgenic cardiomyocytes after basal PDE3 (cilostamide, Cilo) and (C,D) PDE4 (rolipram, Roli) inhibition. IBMX inhibits multiple PDEs, and isoprenaline (ISO) stimulates the $\beta$-adrenergic response leading to maximal FRET change. (E) Quantification of FRET experiments uncovered PDE3 and PDE4 as the PDE families crucial for confining the SERCA2a compartment from the bulk cytosol. Means \pm SE, $n=9-13$ cells from 3-4 hearts per condition. * - significant differences at $p<0.05(\mathbf{F})$ Stimulation of freshly isolated wildtype cardiomyocytes with vehicle, PDE inhibitors (BAY 60-7550, PDE2 inhibition; Cilo, PDE3 inhibition; Roli, PDE4 inhibition; IBMX, total PDE inhibition) or ISO for 15 min. PKA dependent PLN phosphorylation $\left(P^{\text {Ser16 }}\right)$ increased especially after PDE3 and PDE4 inhibition. Bar graphs quantify the immunoblot results. Means $\pm \mathrm{SE}, \mathrm{n}=8-10$ mouse hearts per condition. ${ }^{*}$ - significant differences at $p<0.05$. Substance concentrations were as in Figure 26.

\subsection{FRET measurements in Epac1-camps vs. Epac1-PLN cardiomyocytes after TAC surgery}

To directly address the hypothesis that alterations in subcellular cAMP dynamics are a molecular hallmark of cardiac disease, transverse aortic constriction (TAC) surgery in Epac1camps (cytosolic sensor), Epac1-PLN (SERCA2a targeted sensor) as well as in WT animals 
was performed to induce cardiac hypertrophy. Eight weeks after TAC, heart function was significantly decreased in TAC vs. sham control group as measured by echocardiography (Table 12).

Table 12. Echocardiographic phenotyping of wildtype, Epac1-PLN and Epac1-camps transgenic mice 8 weeks after TAC vs sham surgery. AWThd, anterior wall thickness in diastole. HW/BW, calculated heat-to-body-weight ratio. All other parameters were as described in the legend for Table 11.

\begin{tabular}{|l|l|l|l|l|l|l|}
\hline \multirow{2}{*}{ Parameter } & \multicolumn{2}{|l|}{ Wildtype } & \multicolumn{2}{l|}{ Epac1-PLN transgenic } & \multicolumn{2}{l|}{ Epac1-camps transgenic } \\
\cline { 2 - 7 } & sham & TAC & sham & TAC & sham & TAC \\
\hline Gradient $(\mathrm{mm} \mathrm{Hg})$ & $3.9 \pm 0.5$ & $76.2 \pm 7.1^{*}$ & $6.6 \pm 2.2$ & $92.6 \pm 22.6^{*}$ & $4.9 \pm 1.7$ & $77.2 \pm 9.1^{*}$ \\
FS $(\%)$ & $34.6 \pm 1.5$ & $27.3 \pm 2.2^{*}$ & $41.3 \pm 5.0$ & $28.5 \pm 5.6^{*}$ & $31.3 \pm 7.4$ & $22.7 \pm 6.2^{*}$ \\
FAS (\%) & $55.4 \pm 1.4$ & $46.1 \pm 3.2^{*}$ & $62.7 \pm 5.4$ & $44.0 \pm 10.1^{*}$ & $45.5 \pm 7.7$ & $36.5 \pm 8.9$ \\
EF (\%) & $60.8 \pm 1.2$ & $52.2 \pm 2.9^{*}$ & $68.2 \pm 5.2$ & $51.6 \pm 8.1^{*}$ & $51.5 \pm 7.8$ & $42.8 \pm 7.8$ \\
HW/BW $(\mathrm{mg} / \mathrm{g})$ & $4.2 \pm 0.3$ & $6.6 \pm 0.4^{*}$ & $4.6 \pm 0.6$ & $6.6 \pm 1.1^{*}$ & $4.4 \pm 0.4$ & $6.8 \pm 1.4^{*}$ \\
AWThd $(\mathrm{mm})$ & $0.79 \pm 0.02$ & $0.97 \pm 0.04^{*}$ & $0.85 \pm 0.13$ & $1.09 \pm 0.19^{*}$ & $0.81 \pm 0.07$ & $1.02 \pm 0.17^{*}$ \\
LV-EDD $(\mathrm{mm})$ & $3.8 \pm 0.1$ & $4.0 \pm 0.1^{*}$ & $4.0 \pm 0.3$ & $4.1 \pm 0.3$ & $4.1 \pm 0.3$ & $4.4 \pm 0.1^{*}$ \\
HR & $498 \pm 21$ & $512 \pm 9$ & $389 \pm 57$ & $422 \pm 52$ & $481 \pm 45$ & $466 \pm 39$ \\
$\mathrm{n}$ & 5 & 7 & 13 & 16 & 7 & 8 \\
\hline
\end{tabular}

Besides, TAC surgery did not lead to any visible alteration of Epac1-PLN sensor localisation in TG heart cross sections (Figure 28).
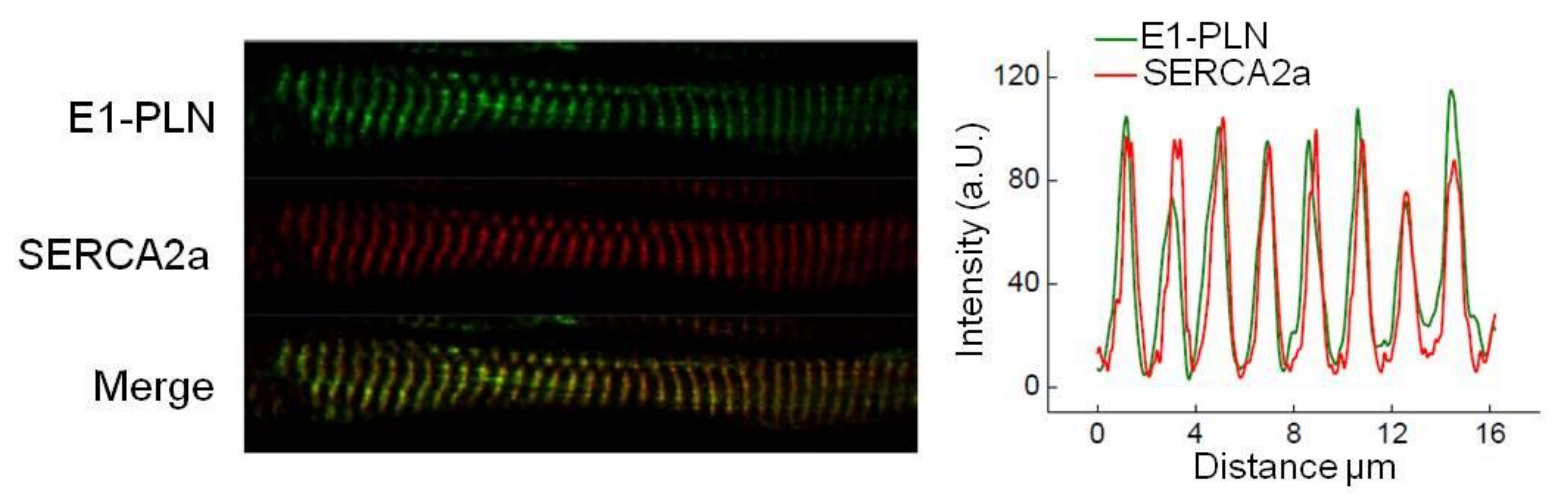

Figure 28. Epac1-PLN localisation is not altered in TAC mice. Confocal image of a representative immunostained transgenic Epac1-PLN heart cross section 8 weeks after TAC surgery. Epac1-PLN and Serca2a showed proper colocalisation as demonstrated by the fluorescence intensity overlay. 
Eight weeks after surgery, cardiomyocytes of sham and TAC treated animals were isolated and used for the following experiments.

\subsubsection{PDE contributions after TAC induced hypertrophy in the bulk cytosol vs. the SERCA2a compartment.}

FRET experiments in diseased cardiomyocytes after $\beta$-adrenergic stimulation with ISO and subsequent treatment with various PDE inhibitors revealed that the strong effects of the PDE4 family in the bulk cytosol (Epac1-camps sensor) were significantly decreased after TAC (Figure 29 A,C,E).

A

C
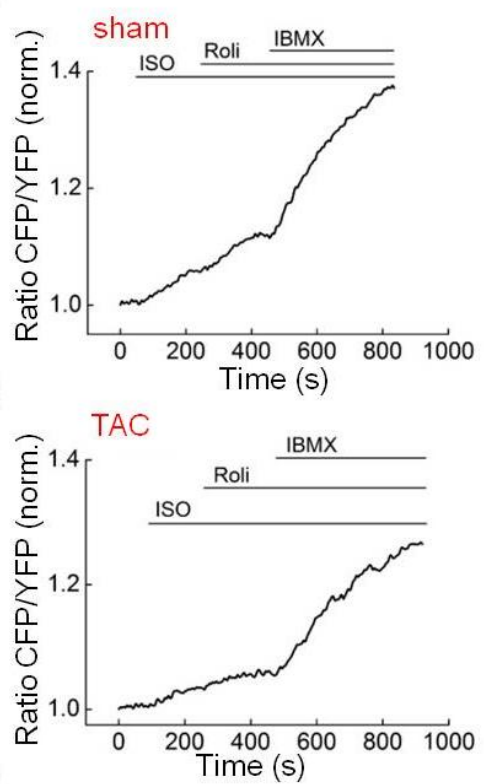

$E$

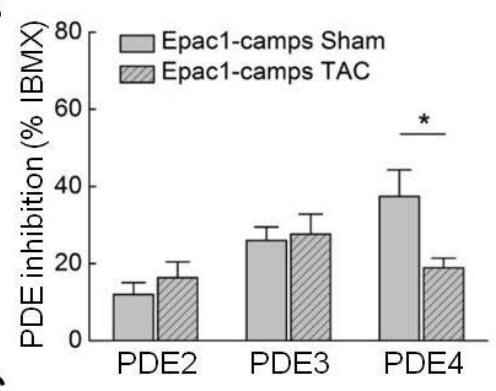

G

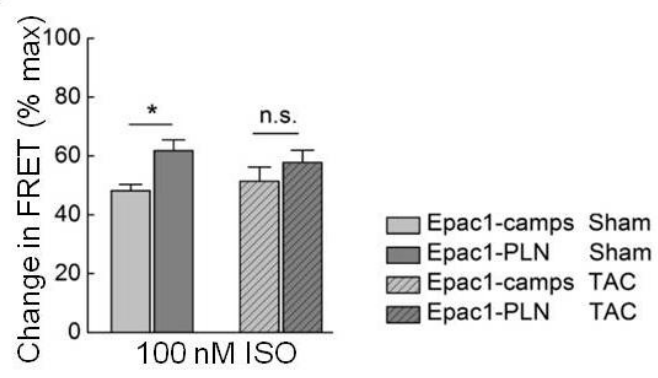

B
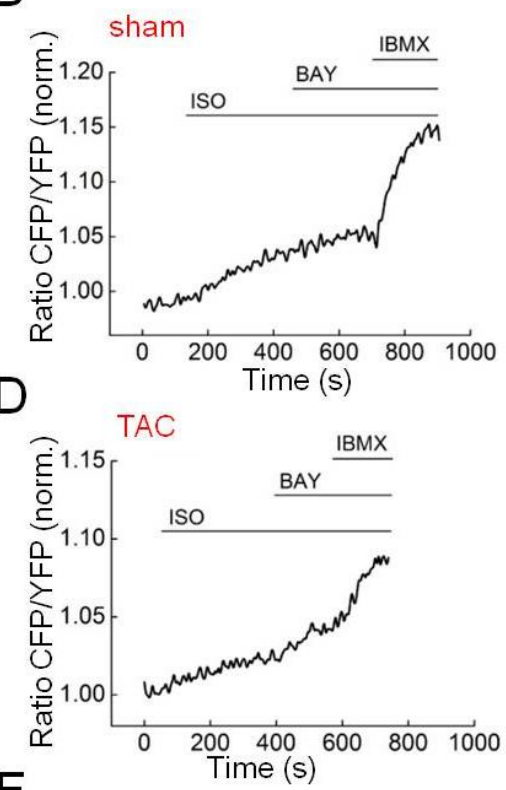

$\mathrm{F}$

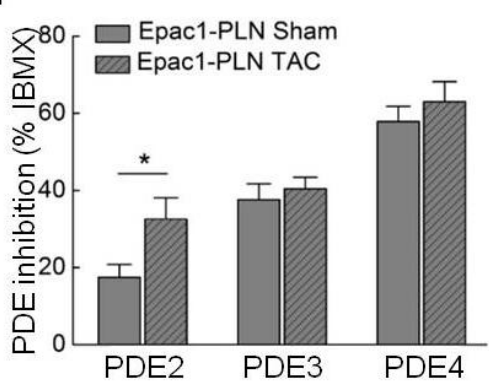

Figure 29. Contributions of PDEs to CAMP hydrolysis after $\beta$-AR stimulation in sham and TAC cardiomyocytes. $\quad(A, C)$ Representative FRET traces from sham and TAC cardiomyocytes expressing Epac1-camps or $(\mathbf{B}, \mathbf{D})$ Epac1PLN, treated with $1 \mathrm{nM}$ ISO for submaximal $\beta-A R$ stimulation and subsequently with PDE4 inhibitor rolipram (Roli, $10 \mu \mathrm{M}$ ) or PDE2 inhibitor (BAY 607550, $100 \mathrm{nM}$ ), respectively. (E) Quantification of cytosolic (Epac1-camps)

FRET experiments revealed a significant decrease of PDE4 contribution after TAC. (F) Quantification of SERCA2a specific (Epac1-PLN) FRET experiments revealed a significant increase of PDE2 contribution in TAC cells, while other PDEs were not affected. PDE3 was inhibited by $10 \mu \mathrm{M}$ cilostamide. (G) Comparison of the magnitudes of ISO responses measured with both sensors in an experiment performed as described in Figure 22E. These data argue for the loss of receptormicrodomain communication after TAC. Means \pm SE, $n=9$ 12 cells from 3-4 mouse hearts per condition. * - significant differences at $p<0.05$. 
This was in line with a previously described reduction of the overall cytosolic PDE4 activity in hypertrophied and failing hearts (Abi-Gerges et al., 2009). In sharp contrast, the PDE4 inhibitor effects in the SERCA2a microdomain (Epac1-PLN sensor) were unchanged after TAC. Instead, there was a significant increase in PDE2 contribution to local cAMP degradation (Figure $29 \mathrm{~B}, \mathrm{D}, \mathrm{F}$ ).

PDEs seem to be important for the communication between $\beta_{1}$-AR and the SERCA2a microdomain (Figure 25). Therefore, the next step was to test whether the change in microdomain-specific PDE-dependent effects after TAC might affect this communication. Sham cells were treated with $100 \mathrm{nM}$ ISO, which led to a stronger FRET change in the SERCA2a microdomain compared to the bulk cytosol, as expected (Figure $29 \mathrm{G}$ and Figure 22). Since the selective $\beta_{2}$-AR stimulation only induced very small FRET changes in the previous experiments (Figure 22), one can assume that the observed FRET changes were almost exclusively $\beta_{1}$-AR dependent. Interestingly, the TAC cells did no longer show a difference between cytosolic (Epac1-camps sensor) and SERCA2a microdomain (Epac1PLN sensor) specific responses to ISO, suggesting that the $\beta_{1}$-receptor-microdomain communication is dramatically impaired in cardiac hypertrophy (Figure $29 \mathrm{G}$ ).
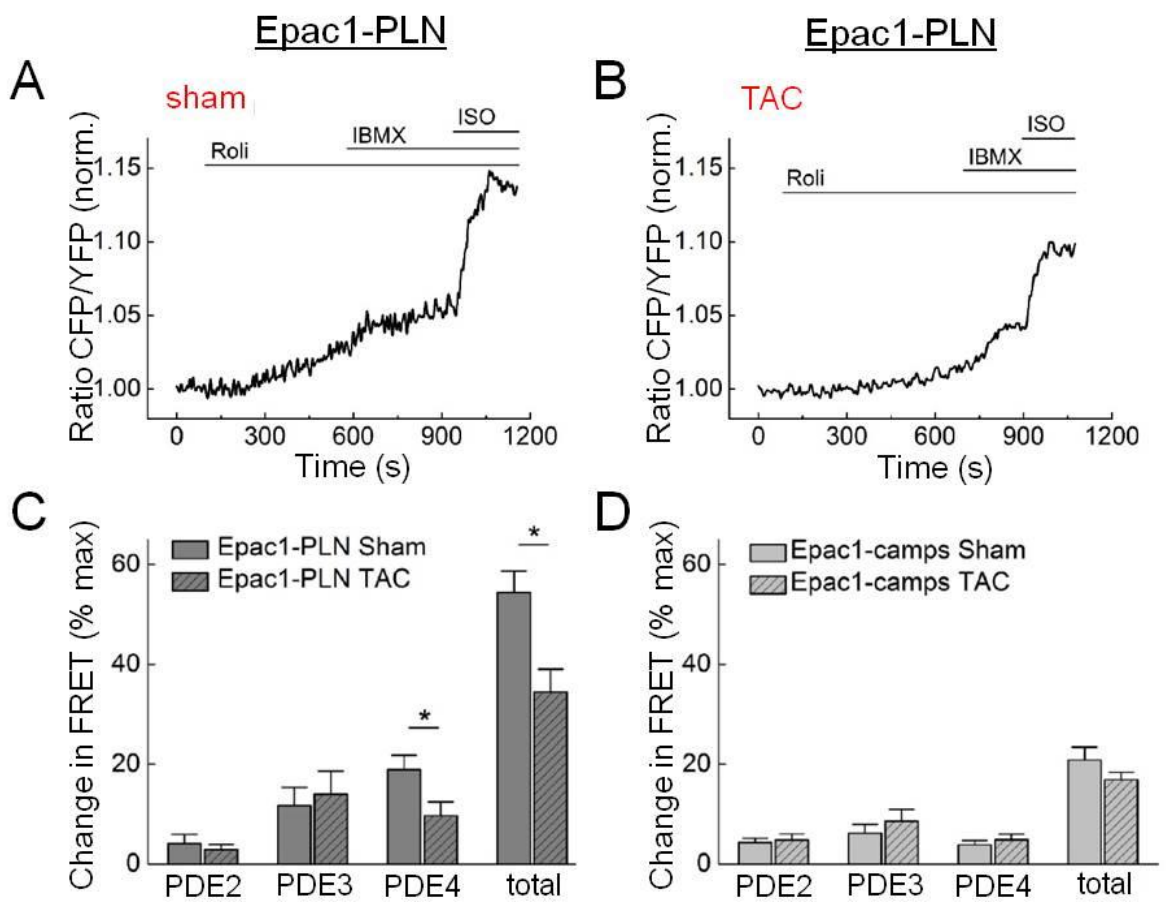

Figure 30. TAC-associated changes in CAMP hydrolysis under basal conditions. Representative FRET traces from sham (A) or TAC (B) Epac1-PLN cardiomyocytes stimulated with the PDE4 inhibitor rolipram (Roli) under basal conditions before maximal stimulation with IBMX and ISO. (C) Quantification of FRET experiments in Epac1-PLN cardiomyocytes revealed a significant decrease of PDE4 contribution to basal cAMP hydrolysis in TAC cells. (D) Quantification of FRET experiments in Epac1-camps cardiomyocytes did not show any change in PDE inhibitor effects. Means \pm SE, $n=7-17$ cells from 3-5 mouse hearts per condition. ${ }^{*}$ - significant differences at $p<0.05$. Compound concentrations were as in Figure 26. 
Surprisingly, basal PDE inhibition in Epac1-camps TAC vs. sham cardiomyocytes did not show any significant differences in any of the tested PDE families (Figure $30 \mathrm{D}$ ) whereas basal PDE4 inhibition in Epac1-PLN TAC cardiomyocytes led to a significant decrease of FRET change (Fig. 30 A-C).

However, in contrast to the FRET data, PDE activity assays in lysates from WT cardiomyocytes did not reveal any changes in whole-cell PDE activities in TAC vs. sham cardiomyocytes (Figure $31 \mathrm{~A}$ ). Furthermore, PDE protein expression levels were not altered after TAC (Figure $31 \mathrm{~B}, \mathrm{C}$ ). These results might imply that alterations of PDE activity at the local microdomain-specific rather than at the whole-cell level occur at this stage of cardiac hypertrophy. Therefore, these changes can be detected only by the targeted FRET sensor approach, as in contrast to cytosolic sensors and classical biochemical techniques.
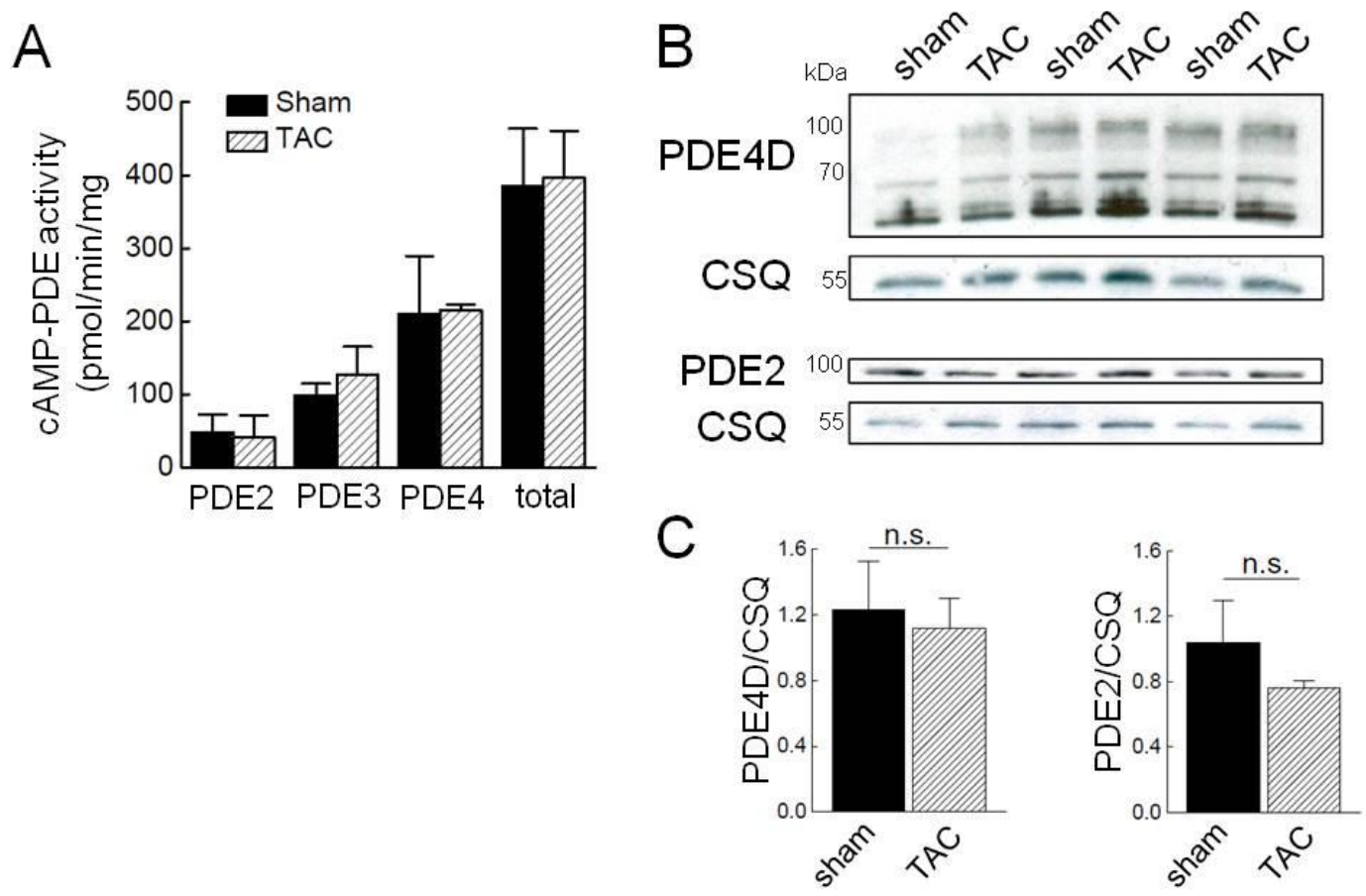

Figure 31. Whole-cell PDE activity and PDE protein levels are not significantly altered in TAC vs. sham mice. (A) CAMP-PDE activities measured in wildtype cardiomyocyte lysates using in vitro PDE activity assays were not significantly different in TAC vs. sham cells (Means $\pm S E, n=2-3$ mice per group). (B) Immunoblot analysis of PDE4D and PDE2A protein levels in wildtype cardiomyocytes (C) Immunoblot quantifications showed no significant alterations of PDE4D and PDE2A protein levels in TAC vs. sham cells (Means $\pm S E, n=3$ hearts per group).

\subsubsection{Calcium handling in TAC cardiomyocytes}

Immunoblot analysis of WT healthy and diseased cardiomyocytes revealed a tendency towards decreased SERCA2a expression levels in TAC vs. sham cells (Figure $32 \mathrm{~A}$ ). This decreased SERCA2a expression was accompanied by significantly slower $\mathrm{Ca}^{2+}$ decline kinetics $\left(\tau_{\mathrm{Ca}}{ }^{2+}\right)$, as a measure of SERCA2a activity in beating cardiomyocytes (Figure $32 \mathrm{~B}$ ), 
suggesting an impaired SERCA2a activity in diseased hearts which cannot be fully compensated by increased local cAMP levels.
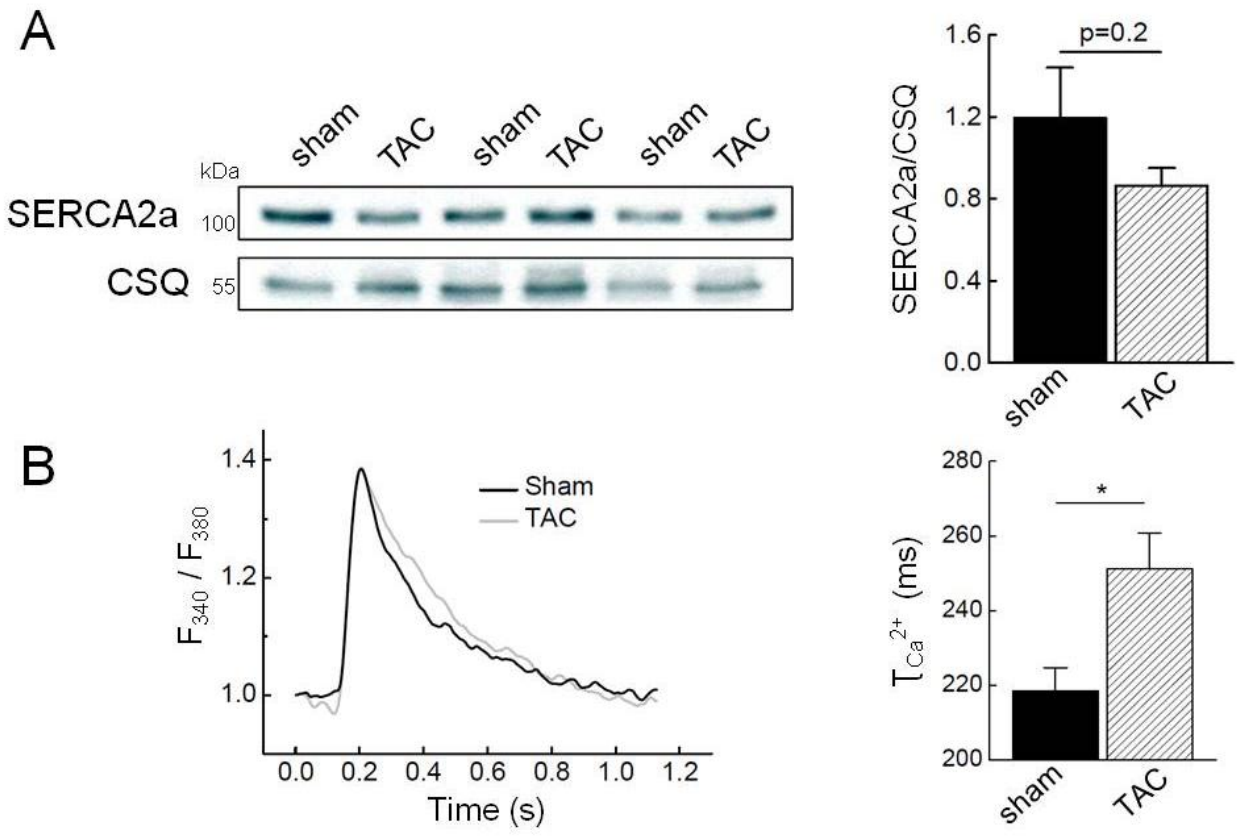

Figure 32. Reduced SERCA2a expression and delayed calcium $\left(\mathrm{Ca}^{2+}\right)$ transient decay in TAC cells. (A) Immunoblot analysis of SERCA2a protein levels in cardiomyocytes revealed a tendency towards lower SERCA2a protein amounts in wildtype TAC vs. sham cells ( $n=3$ hearts per group). (B) $\mathrm{Ca}^{2+}$ transient measurements in beating wildtype cardiomyocytes loaded with Fura-2 revealed an increased $\mathrm{Ca}^{2+}$-decay time constant $\left(\tau_{\mathrm{Ca}}{ }^{2+}\right)$ and thus, impaired diastolic calcium reuptake. Means \pm SE, $n=26-30$ cells from 3 hearts per group, ${ }^{*}$ - significant difference at $p<0.05$.

\subsection{Influence of calcium on global cAMP FRET signals}

Many research groups use FRET based sensors to study cAMP signals in cells like cardiomyocytes. To enhance cell survival and to avoid signalling artefacts due to contraction, these measurements are usually performed under resting conditions. To study the influence of cytosolic $\mathrm{Ca}^{2+}$ fluctuations associated with cardiomyocyte contraction on cAMP dynamics detected by FRET, we measured cytosolic cAMP responses in resting and electrical field stimulated Epac1-camps cardiomyocytes. The pacing protocol led to proper $\mathrm{Ca}^{2+}$ transients in freshly isolated WT cardiomyocytes loaded with Fura-2 (Figure $33 \mathrm{~A}$ ) but no differences in basal FRET ratio intensity were detected between resting and paced Epac1-camps cardiomyocytes (Figure $33 \mathrm{~B}$ ).

In the first step, the effects of contraction on AC activity detected by FRET in Epac1-camps cardiomyocytes were analysed. The cells were stimulated either with ISO to increase the $\beta$ $A R$ dependent cyclase activity (Figure $33 \mathrm{C}$ ) or with the direct $\mathrm{AC}$ activator forskolin (Figure 
$33 \mathrm{E})$. These stimulating agents both led to an increase in cAMP, shown by an increase in the FRET signal which was comparable in its amplitude in resting and paced cardiomyocytes. Upon inhibition of the major murine cAMP-hydrolysing PDE4 family, the cytosolic CAMP levels increased even further, while the subsequent addition of the nonselective PDE inhibitor IBMX had only minor additional effects. No significant difference between control and paced cardiomyocytes regarding PDE4 contribution to total PDE inhibition were detected, regardless of whether we stimulated them with ISO or with forskolin (quantifications shown in Figure $33 \mathrm{D}$ and $\mathrm{F}$, respectively). These data suggest that rapid changes in intracellular $\mathrm{Ca}^{2+}$ during contraction do not significantly affect the AC derived cAMP detection via FRET.

A
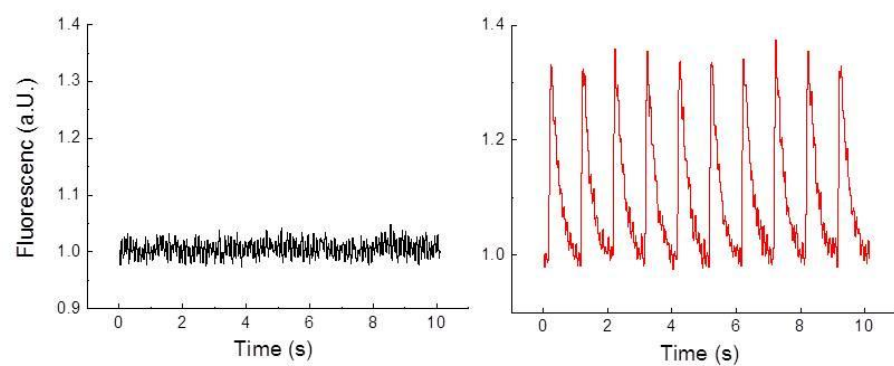

C

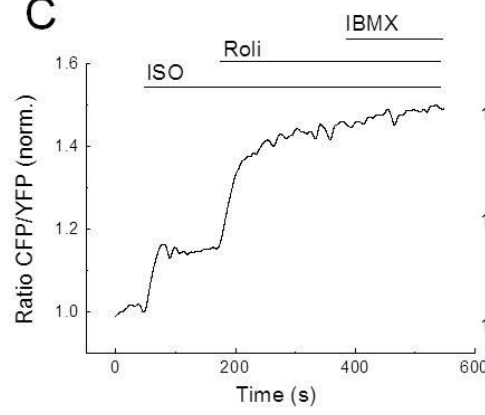

E

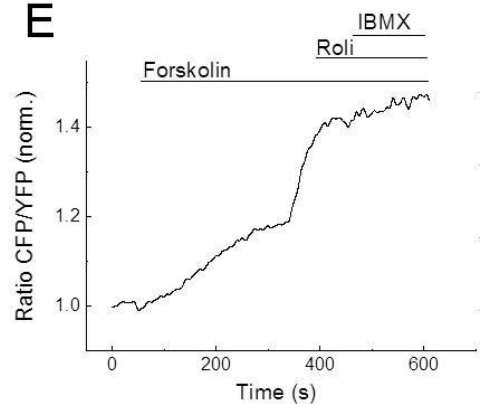

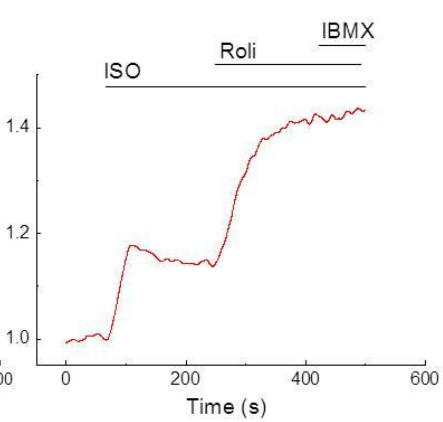

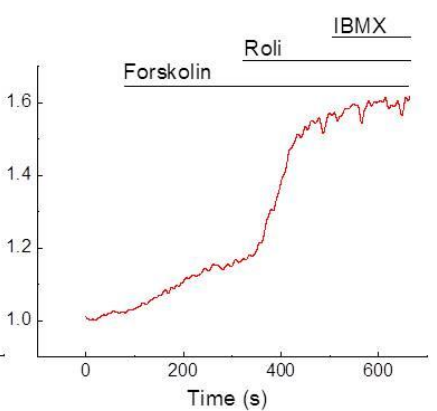

B

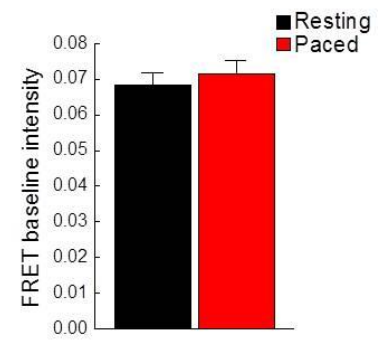

D

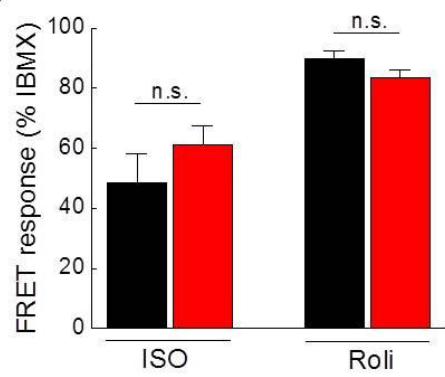

$\mathrm{F}$

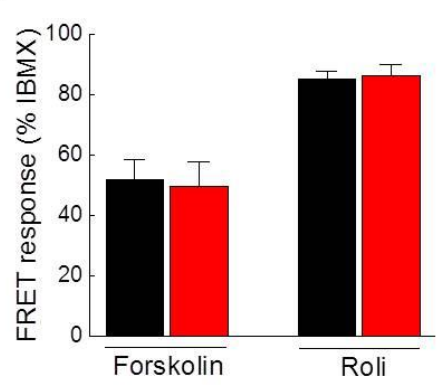

Figure 33. CAMP dynamics in adult mouse ventricular cardiomyocytes upon treatment with cAMP elevating reagents and PDE4 inhibition. (A) Representative calcium traces in Fura-2 loaded WT cardiomyocytes under resting conditions and upon electric field stimulation at $1 \mathrm{~Hz}$ with 20-30 V, respectively. (B) Pacing does not lead to altered basal FRET ratios between resting and field stimulated Epac1-camps cardiomyocytes. (C) Representative FRET traces from Epac1-camps cardiomyocytes stimulated with the $\beta$-AR agonist isoproterenol (ISO, $100 \mathrm{nM}$ ) or (E) the direct AC activator forskolin $(10 \mu \mathrm{M})$ which both lead to an increase of cAMP visualized as an increase in the FRET ratio. Inhibition of PDE4 by rolipram (Roli, 10 $\mu \mathrm{M}$ ) strongly enhances this effect, whereas the

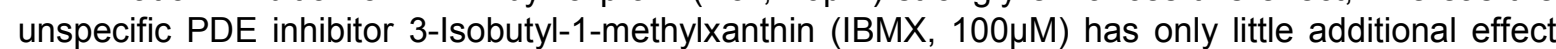
after rolipram application. (D) and (F) Quantification of FRET results reveal no significant differences in FRET changes between control and paced cardiomyocytes. Shown are the means \pm SE $(n=6$ cells from 3 mouse hearts per condition; * - significant difference at $p<0.05$ ) 
PDE1 hydrolytic activity towards CAMP is known to be stimulated by $\mathrm{Ca}^{2+}$ via calmodulin (Sonnenburg et al., 1993), so that a potential decrease of cAMP levels upon $\mathrm{Ca}^{2+}$ elevation can be expected. Therefore it was important to investigate whether $\mathrm{Ca}^{2+}$ fluctuations associated with contraction have any influence on PDE1 activity dependent cAMP signals detected in FRET experiments. Therefore, measurements similar to those shown in Figure 33 were performed, but using PDE1 inhibitor 8-methoxymethyl-3-isobutyl-1-methylxanthine (8-MMX) (Figure 34). Again, no significant differences in the amplitude or kinetics of the FRET signals between control and paced cardiomyocytes stimulated with ISO have been detected, suggesting no effect of rapid $\mathrm{Ca}^{2+}$ transients on the overall PDE1 activity upon $\beta$ adrenergic stimulation detected by FRET (Figure $34 \mathrm{~A}, \mathrm{~B}$ ). Interestingly, stimulation of the cells with the direct $A C$ activator forskolin elevated PDE1 contribution to overall PDE inhibition significantly in field stimulated compared to resting cardiomyocytes. This effect was mimicked by cardiomyocyte preincubation with $\mathrm{Ca}^{2+}$ elevating substances thapsigargin and calcium ionophor (Figure 34 C,D).
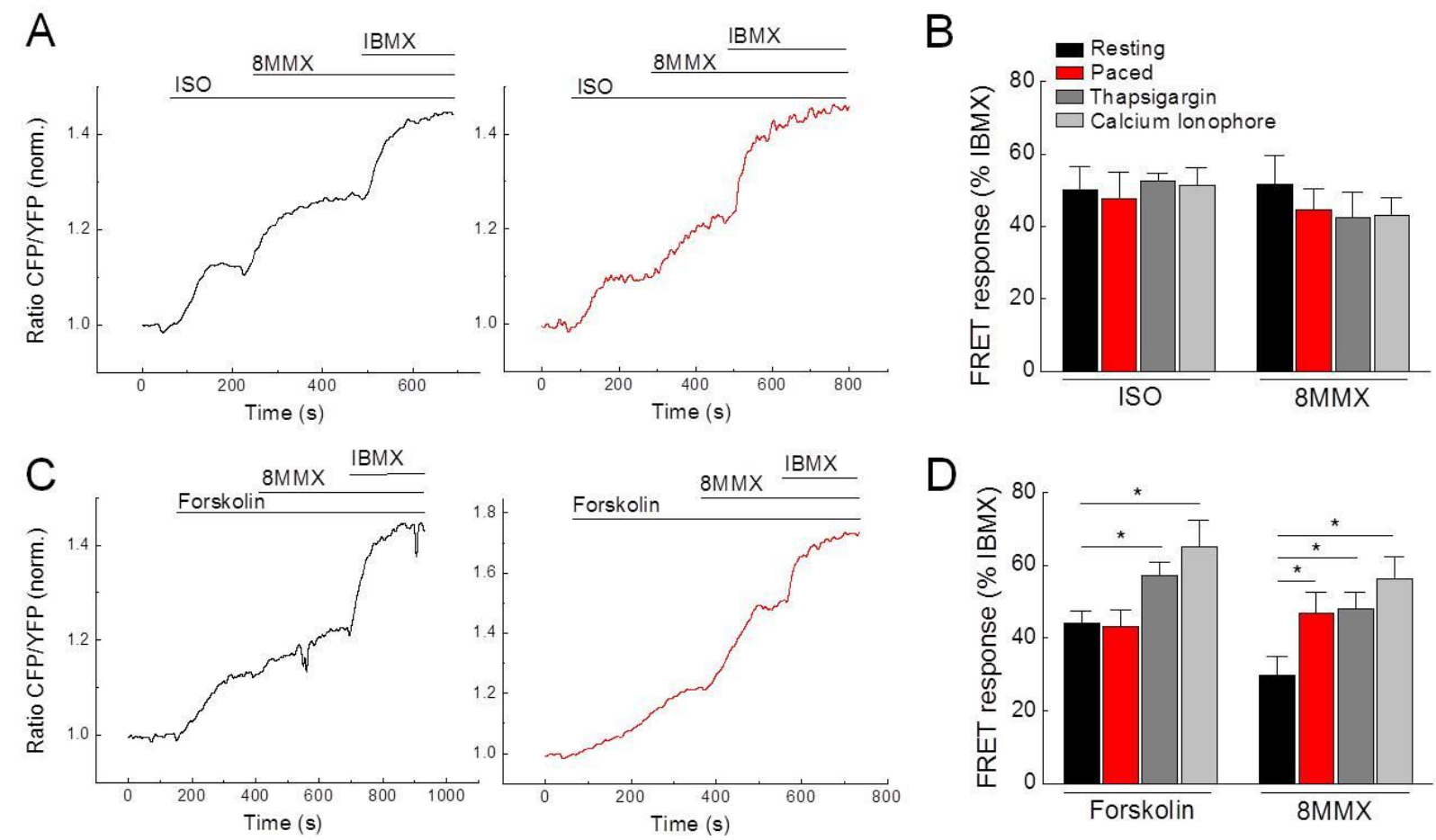

Figure 34. CAMP dynamics in adult mouse cardiomyocytes upon treatment with cAMP elevating agents and PDE1 inhibition. (A) Representative FRET traces of Epac1-camps cardiomyocytes after stimulation with the $\beta$-AR agonist isoproterenol (ISO, 100nM) or (C) the direct AC activator forskolin $(10 \mu \mathrm{M})$ leads to an increase of cAMP visualized as an increase in FRET ratio. Inhibition of PDE1 via 8-MMX $(30 \mu \mathrm{M})$ enhances this effect. Stimulation with the unspecific PDE inhibitor 3-Isobutyl-1-methylxanthin (IBMX, $100 \mu \mathrm{M}$ ) leads to a further increase of cAMP. (B,D), Quantification of experiments shows no significant difference in FRET responses between control and paced cardiomyocytes stimulated with ISO. Forskolin stimulated cardiomyocytes show significant differences in PDE1 contribution to total PDE inhibition in paced compared to resting cells. Pretreatment with calcium elevating reagents such as thapsigargin $(100 \mathrm{nM})$ and calcium ionophore $(10 \mu \mathrm{M})$ resemble the effect of field stimulation. Cells were passed at $1 \mathrm{~Hz}$ and $20-30 \mathrm{~V}$. Means $\pm \mathrm{SE}$ $(n=6-10$ cells from 2-3 mouse hearts per condition; * - significant difference at $p<0.05$ ) 


\section{Discussion}

\subsection{Successful generation of transgenic Epac1-PLN mice}

To measure cAMP dynamics under real time conditions with high temporal and spatial resolution, a TG cAMP reporter mouse line namely CAG-Epac1-camps expressing the cytosolic Epac1-camps FRET biosensor was initially developed (Calebiro et al., 2009). This sensor mouse allows the investigation of global cAMP changes in the bulk cytosol under physiological conditions in living tissues and single cells such as cardiomyocytes.

Today, cAMP compartmentation is a well-accepted paradigm (Fischmeister et al., 2006). Therefore, it is essential to generate subcellular cAMP probes to get a deeper understanding of the molecular mechanisms that regulate the compartmentation of this universal second messenger. Although the already existing targeted cAMP FRET biosensors provided important insights into the molecular mechanisms behind cAMP compartmentation, these probes were either expressed in HEK293A cells, neonatal cardiomyocytes or adult rat cardiomyocytes via virus transduction (Sprenger and Nikolaev, 2013).

Alterations in SERCA2a activity are known to be associated with cardiac disease (Hasenfuss et al., 1994; Nagai et al., 1989) and are probably connected with a complex modification of CAMP/ PKA dependent SERCA2a regulation. To provide a more physiologically relevant system for the detection of subcellular cAMP dynamics, a novel TG mouse model expressing a heart specific SERCA2a targeted FRET based CAMP biosensor, namely Epac1-PLN, was generated within this $\mathrm{PhD}$ project. The novel sensor mouse enables the detection of local cAMP dynamics with high temporal and spatial resolution in adult cardiomyocytes. Besides, TG cardiomyocytes are freshly isolated and can be used for FRET measurements immediately after cell isolation. This excludes cultivation artefacts due to adenoviral sensor transduction and allows cAMP measurements under more physiological conditions. Importantly, this novel Epac1-PLN sensor mouse can be combined with various genetic and experimental disease models and thus enables the study of altered cAMP signalling under pathological conditions such as cardiac hypertrophy induced by TAC surgery.

The first SR-specific FRET measurements were performed by Liu and co-workers that used the AKAR3 PKA activity reporter (Zhang et al., 2001) and the Epac based ICUE3 cAMP sensor (Sample et al., 2012) fused to the transmembrane domain of PLN (Liu et al., 2012; Liu et al., 2011). In this thesis, the already published cytosolic Epac1-camps FRET biosensor (Nikolaev et al., 2004) was also either fused to a truncated ( $\triangle 1-18$ PLN, including the transmembrane domain) or full length version of PLN (Figure 9). This led to the localisation 
of the sensor constructs to the SERCA2a microdomain. Analysis of both sensors in HEK293A cells revealed stronger FRET changes in the full length compared to the truncated sensor version. Therefore, the full length PLN based Epac1-PLN sensor was used for further experiments and for the generation of TG mice. Besides, the use of full length PLN was believed to sustain the physiology within the SERCA2a microdomain, whereas a truncated version might insert unwished artificiality to this compartment, for example because it can neither be PKA-phosphorylated nor form pentamers as the endogenous PLN does. Importantly, one might speculate that PKA dependent phosphorylation of the sensor PLN might lead to intramolecular rearrangement resulting in FRET changes independent of CAMP binding. However, this was not the case for the Epac1-PLN construct (Figure 10) so one can be sure that the detected FRET changes truly represent cellular CAMP dynamics.

To analyse cAMP compartmentation within adult ventricular cardiomyocytes, an $\alpha-\mathrm{MHC}$ based Epac1-PLN sensor construct was cloned and used for the generation of the novel TG sensor mouse line (Figure 11). The a-MHC promoter allows cardiomyocyte specific expression of the sensor construct, thereby avoiding undesirable side effects due to needless global sensor expression.

To be prepared for unexpected problems in TG mouse line generation, an Epac1-PLN adenovirus for transduction of rat cardiomyocytes was generated as an alternative strategy (Figure 12). Proper FRET responses to ISO induced cAMP increases were detected in adult rat cardiomyocytes transduced with this adenovirus. Therefore, the adenoviral approach would have been a possible alternative to the use of TG Epac1-PLN mouse cardiomyocytes. Anyway, as already mentioned, cultivation of adult cardiomyocytes can be associated with experimental artefacts and therefore the TG mouse line was the favoured tool during the following experiments within this work.

The novel TG mouse line showed Epac1-PLN sensor expression in virtually all cardiomyocytes. Besides, the sensor showed proper co-localisation with SERCA2a in stained heart cross sections and in heart fractions (Figure 14), thereby complying with the basic requirement for SERCA2a microdomain specific CAMP measurements. Epac1-PLN TG hearts showed a two-fold overexpression of PLN on the mRNA level (Figure $18 \mathrm{~A}$ ) which is also common for other PLN overexpressing mouse lines (Kiriazis and Kranias, 2000). Importantly, no adverse effects on cardiac morphology and function were detected in TG animals (Figure 15). Earlier PLN TG mouse models did also not show any severe cardiac phenotype apart from a slightly depressed systolic function at similar amounts of PLN overexpression. This effect was explained by the increased inhibition of SERCA2a function due to PLN overexpression (Kadambi et al., 1996; Kiriazis and Kranias, 2000). Surprisingly, cardiac function was slightly elevated in the TG Epac1-PLN animals compared to their WT littermates (Table 11). This unexpected increase in heart function might be due to some 
compensatory mechanisms associated with altered $\mathrm{Ca}^{2+}$ handling, similarly to the situation observed in a mouse model with overexpression of a PLN mutant which cannot be PKA phosphorylated (Brittsan et al., 2003). For example, Epac1-PLN TG cardiomyocytes showed a tendency of increased NCX expression (Figure $19 \mathrm{~A}, \mathrm{~B}$ ) which might allow faster $\mathrm{Ca}^{2+}$ extrusion at the end of each systole, thereby promoting lusitropy (cardiac relaxation). PLN overexpression in TG Epac1-PLN cardiomyocytes was also shown to be associated with SERCA2a overexpression. This would also lead to faster $\mathrm{Ca}^{2+}$ extrusion from the cytosol into the SR and an increase in inotropy (cardiac contractility) and lusitropy. However, the PLN/ SERCA2a ratio in WT vs. TG cardiomyocytes was not altered (Figure $18 \mathrm{~B}-\mathrm{D}$ ), again pointing towards a compensatory mechanism to balance the enhanced SERCA2a inhibition by the TG sensor PLN. Besides, $\mathrm{Ca}^{2+}$ imaging showed almost unaltered $\mathrm{Ca}^{2+}$ decline kinetics in WT vs. TG Epac1-PLN cardiomyocytes in the absence and presence of ISO stimulation. Additionally, SR- $\mathrm{Ca}^{2+}$ load was unchanged which might be explained by the stable PLN/ SERCA2a ratio in WT vs. TG animals. Due to this steady PLN/ SERCA2a ratio one can assume that, although SERCA2a is upregulated in TG Epac1-PLN cardiomyocytes, microdomain specific cAMP measurements with this FRET sensor are still reliably recorded within the SRECA2a microdomain.

Endogenous PLN exists in a monomeric and a pentameric form (Kimura et al., 1997; Zvaritch et al., 2000) and also the Epac1-PLN sensor (monomer $\sim 77 \mathrm{kDa}$ ) is able to form oligomers either with itself or the endogenous PLN (Figure $16 \mathrm{~A}$ ). Besides, Epac1-PLN mimicked endogenous PLN for PKA phosphorylation $\left(\mathrm{P}^{\text {Ser16 }}\right)$ upon stimulation with $100 \mathrm{nM}$ ISO without affecting endogenous PLN phosphorylation (Figure 16 B-D). Additionally, the sum of total basal PKA and CaMKII dependent phosphorylation of PLN was not altered in WT vs. TG cardiomyocytes. These results assure that a functionally normal PLN was introduced via Epac1-PLN expression and that in TG Epac1-PLN cardiomyocytes, the function and molecular composition of the SERCA2a microdomain is preserved.

However, there is controversy about the nature of PLN dependent SERCA2a regulation. It has been believed that upon PKA dependent phosphorylation, PLN monomers dissociate from SERCA2a to form pentamers, thereby relieving its inhibition on the $\mathrm{Ca}^{2+}$ pump (Chen et al., 2010; James et al., 1989; Mueller et al., 2004). On the other hand, PLN pentamers have also been shown to inhibit SERCA2a function (Zhai et al., 2000). Latest studies revealed that active SERCA2a binds to fully phosphorylated PLN (Dong and Thomas, 2014) and that PLN exists in a conformational equilibrium between different, phosphorylation dependent intramolecular states that differ in their SERCA2a inhibition characteristics (Gustavsson et al., 2013). Therefore, SERCA2a inhibition might be mediated due to phosphorylation dependent conformational changes within the SRECA2a-PLN complex rather than by dissociation of PLN. These results indicate that Epac1-PLN monomers as well as Epac1- 
PLN pentamers are tightly targeted to SERCA2a, thereby allowing reliable measurements of local SERCA2a associated cAMP dynamics with the Epac1-PLN sensor construct.

\subsection{Localised FRET measurements reveal differentially regulated cAMP dynamics in} healthy Epac1-camps and Epac1-PLN cardiomyocytes

To understand the molecular mechanisms that confine the SERCA2a microdomain from the bulk cytosol, cytosolic cAMP FRET measurements have been performed in TG Epac1camps cardiomyocytes and compared to SERCA2a localised CAMP dynamics measured in TG Epac1-PLN cardiomyocytes. Several differences have been observed that are discussed in the following sections.

\subsubsection{Local PDE effects are essential for channelling cAMP from the $\beta 1-A R$ to the SERCA2a microdomain}

Recently, it has been shown that $\beta_{1}$-AR stimulation leads to far-reaching cAMP signals within cardiomyocytes whereas $\beta_{2}$-AR signals remain locally confined (Nikolaev et al., 2006). Besides, $\beta_{2}$-AR stimulation has been shown to have no effect on PLN phosphorylation (Kuschel 1999). These results are in line with the findings in Epac1-PLN TG cardiomyocytes. Here, specific $\beta_{2}$-AR stimulation has almost no effect on the FRET signal in the SERCA2a compartment (Figure 22) which lies deep within the cell reflecting the highly compartmentalized $\beta_{2}$-AR associated cAMP dynamics under the control of PDE4D5 (Richter et al., 2008).

However, $\beta_{1}$-AR stimulation of freshly isolated Epac1-camps and Epac1-PLN TG cardiomyocytes showed significantly stronger FRET changes in the SERCA2a compartment when normalized to the maximal FRET response, although the used ISO concentrations should have been almost saturating for both sensors (Figure 22 and Figure 24). In fact, Epac1-PLN seems to be more sensitive to lower ISO concentrations than Epac1-camps although having a slightly lower affinity for cAMP (Figure 24). Recently, it has been shown that PDE4D8 is associated with the $\beta_{1}-A R$, thereby regulating cAMP signalling around this receptor (Richter et al., 2008). Since the expression of PDE4D8 in WT vs. Epac1-PLN TG cardiomyocytes is not altered (Figure $20 \mathrm{~A}, \mathrm{C}$ ), one can exclude the possibility that increased CAMP sensing in the SERCA2a compartment is due to decreased PDE4D8 expression at the $\beta_{1}$-AR. This indicates that a specific cAMP compartment deep inside the cell might have privileged access to and preferentially communicates with the receptor, thereby sensing higher cAMP levels compared to the bulk cytosol. This intriguing possibility can be described 
as a "channelling" of cAMP from the surface membrane to its downstream targets such as PKA in the subcellular SERCA2a microdomain.

To avoid measurement artefacts due to cardiomyocyte contraction, FRET experiments were performed without field stimulation although it is known that $\mathrm{Ca}^{2+}$ is able to influence cAMP dynamics during cardiac contraction (Wikman-Coffelt et al., 1983). Interestingly, experiments in field stimulated Epac1-PLN cardiomyocytes did not differ from those under resting conditions (Figure 23), which might be due to insufficient sensitivity of the Epac1-PLN sensor for sensing such rapid fluctuations. However, the absent differences between paced and resting Epac1-PLN cardiomyocytes justify the favoured use of resting cells in the FRET experiments.

In HEK293A cells, it has been shown that upon PGE $_{1}$ stimulation, PDEs, rather than acting as diffusion barriers (Jurevicius and Fischmeister, 1996), act as local sinks that drain cAMP to specific compartments (Terrin et al., 2006). This would allow the coexistence of multiple cAMP microdomains independent of their distance from the site of cAMP synthesis. Indeed, if PDE3 and PDE4 were blocked at the same time, the previously detected differences in the $\beta$-adrenergic response between the bulk cytosol and the SERCA2a compartment were abolished (Figure $25 \mathrm{~A}-\mathrm{C}$ ). The same was detected when PDEs were blocked with the unselective inhibitor IBMX (Figure $25 \mathrm{D}, \mathrm{E}$ ). This reinforces the hypothesis that cytosolic PDEs, especially PDE3 and PDE4, may somehow channel the cAMP from its site of synthesis into a compartment deep within the cell and simultaneously prevent cAMP diffusion into the cytosol. This would allow cAMP to perform its signalling effect directly at its specific PKA targets. One might also speculate about the involvement of $A C$ signalling on internalized vesicles (Calebiro et al., 2009) or the activation of soluble ACs deep inside the cells (Chen et al., 2012). At the same time, cytosolic PDEs might also act as cAMP sinks which keep cytosolic cAMP concentrations relatively low. However, the exact molecular mechanisms behind this phenomenon remain to be defined.

\subsubsection{PDE4 plays the predominant role in restricting CAMP signals after $\beta$-AR stimulation and under basal conditions}

Upon $\beta$-AR stimulation, PDE4 inhibition led to the strongest FRET change in both the bulk cytosol and in the SERCA2a compartment, followed by PDE3 and PDE2 inhibition. This might lead to the assumption that there is a certain hierarchy in PDE contribution to shaping cAMP signals connected with $\beta$-AR stimulation (PDE4 > PDE3 > PDE2). However, PDE4 contribution after ISO treatment was significantly stronger in the cytosol compared to the SERCA2a compartment (Figure 26). This indicates a crucial role of PDE4 under conditions with high CAMP concentrations to avoid excessive diffusion of cAMP to other subcellular 
locations and potentially detrimental cAMP spillover. Over the last few years, PDE4 has been reported to play a critical role in cAMP compartmentation. For instance, the RyR2 signalling complex has been shown to be associated with PDE4D3 (Lehnart et al., 2005). Besides, PDE4D8 and PDE4D5 could be linked to $\beta_{1}$ - and $\beta_{2}$-ARs signalling complexes, respectively (Richter et al., 2008). Together with these results, the findings within this $\mathrm{PhD}$ thesis suggest a major role of PDE4 in generating multiple subcellular cAMP microdomains. Under basal conditions, PDEs are most important for confining and protecting the subcellular compartments from basal cytosolic cAMP levels ( 1 $\mu \mathrm{M}$ (lancu et al., 2008)) to avoid inappropriate PKA substrate phosphorylation. In this thesis, first direct evidence is provided that under basal conditions without $\beta-A R$ stimulation, PDE3 and especially PDE4 families play a critical role in confining the SERCA2a microdomain from the bulk cytosol to limit basal PLN phosphorylation (Figure 27). Studies in neonatal rat cardiomyocytes expressing a targeted PKA activity reporter AKAR3 fused to PLN recently showed that PKA activity in this microdomain is also lower and controlled by PDE4 under basal conditions (Liu et al., 2011). Besides, studies using $\mathrm{PDE} 3 \mathrm{~A}^{-/-}$and $\mathrm{PDE} 3 \mathrm{~B}^{-/-}$mice showed that PDE3A interacts with SERCA2a thereby regulating basal myocardial contractility (Beca et al., 2013). Inhibition of PDE3 in human hearts increases cardiac contractility, relaxation and diastolic function (Colucci, 1991; Osadchii, 2007). These observations are consistent with the findings of this $\mathrm{PhD}$ thesis that PDE3 is crucial for the regulation of baseline cAMP dynamics around the SERCA2a compartment in healthy hearts. PDE3 inhibition would induce a local increase of cAMP and subsequent PKA dependent phosphorylation of PLN (Figure $27 \mathrm{~F}$ ), thereby leading to increased SERCA2a function and contraction. However, in end stage heart failure patients, PDE3 inhibitors provide only short term benefit whereas prolonged treatment leads to increased mortality, primarily as a result of arrhythmias and sudden death (Movsesian et al., 2009; Nony et al., 1994; Packer et al., 1991).

Studies in PDE4D ${ }^{-1-}$ mice could identify PDE4D as a critical regulator of baseline SR $\mathrm{Ca}^{2+}$ release via PDE4D co-immunoprecipitation with SERCA2a and by investigating $\mathrm{Ca}^{2+}$ transients and PLN phosphorylation status (Beca et al., 2011). Inhibition of PDE4 and ablation of PDE4D in mice elevated contractility, SR $\mathrm{Ca}^{2+}$ loads, $\mathrm{Ca}^{2+}$ transients and PLN phosphorylation (Beca et al., 2011; Kerfant et al., 2007). These results are supported by the findings in this PhD thesis that show increased cAMP signals after PDE4 inhibition in TG Epac1-PLN cardiomyocytes under basal conditions (Figure 27) which again should enhance SERCA2a activity due to increased PLN phosphorylation (Figure $27 \mathrm{~F}$ ). Interestingly, total PDE4 expression is not increased in Epac1-PLN TG cardiomyocytes compared to WT cardiomyocytes (Figure $20 \mathrm{~A}, \mathrm{~B}$ ), although SERCA2a levels are elevated in the TG cells (Figure $18 \mathrm{~B}, \mathrm{C}$ ). Because PDE4 has been shown to be critically involved in the regulation of basal CAMP levels within the SERCA2a microdomain, one might assume that the relative 
PDE4 amount in the SERCA2a microdomain of Epac1-PLN TG cardiomyocytes is slightly lower than in WT cells. Therefore, the basal PDE4 inhibition effect on the SERCA2a associated CAMP levels might be even stronger than those observed in the Epac1-PLN FRET experiments. However, the results gained in the FRET experiments could be confirmed in immunoblot analysis by investigating basal PLN phosphorylation after selective PDE inhibition which was significantly increased after PDE3 and PDE4 inhibition (Figure 27 F). Interestingly, the group around Rudolphe Fischmeister showed increased basal PLN phosphorylation only after PDE4 inhibition combined with PDE3 inhibition. One explanation for this discrepancy might be that their results were gained in adult rat ventricular myocytes and the amount of PLN phosphorylation was normalized to cardiac calsequestrin expression instead of total PLN. Besides, they analysed PLN pentamer phosphorylation whereas in this PhD thesis PLN monomer phosphorylation after sample denaturation was analysed. However, together with these findings, the data gained in this $\mathrm{PhD}$ thesis indicate that PDE3 and PDE4 are both involved in the regulation of basal cAMP dynamics in the SERCA2a microdomain and therefore in the regulation of heart contraction due to modulation of PLN phosphorylation.

To extend these studies in the future, PDE3- and PDE4- isoform selective inhibitors (Houslay et al., 2005) or crossing Epac1-PLN mice with specific PDE3A ${ }^{-1-}$ PDE4D ${ }^{-1-}$ mice would be of great benefit.

\subsection{Localised FRET measurements reveal differentially regulated cAMP dynamics in diseased Epac1-camps and Epac1-PLN cardiomyocytes}

Cardiac hypertrophy may ultimately lead to heart failure. Therefore, it was interesting to understand the molecular mechanisms behind this disease to identify possible targets for intervention in disease progression. Changes in local cAMP signalling are supposed to be a molecular hallmark of cardiac disease such as hypertrophy. To address this hypothesis, Epac1-camps, Epac1-PLN and WT mice were subjected to TAC surgery to induce cardiac hypertrophy.

This $\mathrm{PhD}$ project provides the first direct insights into alterations of local cAMP dynamics at SERCA2a in cardiac hypertrophy. Despite the slightly increased heart function of TG Epac1PLN mice, animals developed a significant reduction of cardiac performance 8 weeks after TAC, confirming that Epac1-PLN mice are a valuable tool to investigate cAMP dynamics in cardiac hypertrophy. Also Epac1-camps mice showed significant reduction in heart function after TAC surgery. For instance, a pronounced hypertrophy was indicated by a significant increase in HW/BW ratio and AWThd and significantly diminished heart function as demonstrated by the changes in FS, FAS and EF. Besides, LV-EDD values suggest a slight 
ventricular dilatation which is typical for decompensated hypertrophy and heart failure (Diwan and Dorn, 2007) (Table 12). Therefore, the TAC model used in this PhD thesis provides an insight into cardiac hypertrophy with a tendency for transitioning to heart failure. However, Epac1-PLN sensor was still localised to SERCA2a in TAC treated animals allowing reliable cAMP measurements in the diseased cardiomyocytes (Figure 28).

\subsubsection{After $\beta$-AR stimulation, PDE4 and PDE2 contributions are altered in TAC treated cardiomyocytes from Epac1-camps and Epac1-PLN mice, respectively.}

In cardiomyocytes from TAC treated animals, PDE4 contribution after $\beta$-AR stimulation was shown to be significantly decreased in the cytosol (Epac1-camps sensor) (Figure 29 A,C,E). This is consistent with results gained in rats with compensated hypertrophy (Abi-Gerges et al., 2009) where the activity of both PDE4A and PDE4B was significantly reduced.

In sharp contrast to the results gained in Epac1-camps cardiomyocytes, PDE2 inhibition after $\beta$-AR stimulation in Epac1-PLN cardiomyocytes led to a significantly higher FRET change in the SERCA2a compartment in TAC vs. sham control cells (Figure $29 \mathrm{~B}, \mathrm{D}, \mathrm{F}$ ). This indicates an increased contribution of PDE2 to cAMP hydrolysis in the SERCA2a compartment during hypertrophy. Recently, it has been shown that PDE2 is markedly upregulated in end stage failing human hearts, thereby blunting catecholamine responsiveness (Mehel et al., 2013). Besides, a dramatic upregulation of PDE2 has been observed on the whole-cell level in various models of chronic cardiac disease. Additionally, diseased rat cardiomyocytes transduced with the Epac2-camps FRET sensor (Nikolaev et al., 2004) revealed a global increase in PDE2 contribution to cAMP hydrolysis (Mehel et al., 2013). However, in this PhD thesis, no difference in PDE2 contribution to CAMP degradation in the bulk cytosol of healthy vs. diseased Epac1-camps cardiomyocytes was observed (Figure 29 E). This aberration might be due to the different disease models used (rats subjected to chronic ISO infusion vs. mice after TAC surgery) and cultivation of rat cardiomyocytes during Epac2-camps virus transduction vs. freshly isolated TG cardiomyocytes. Mehel et al. also analysed PLN phosphorylation in PDE2 overexpressing cardiomyocytes, uncovering a significant decrease of PKA associated PLN phosphorylation after ISO stimulation but no effect under basal conditions. These results are consistent with the findings that PDE2 inhibition in hypertrophied Epac1-PLN cardiomyocytes induced a FRET change exclusively upon ISO stimulation and not under basal conditions (Figure $29 \mathrm{~F}$ and Figure $30 \mathrm{C}$ ). This might indicate a mechanism by which local PDE2 upregulation compensates for decreased PDE4 contribution to cAMP degradation, thereby protecting the heart and especially the SERCA2a compartment from cAMP overflow and excessive PLN phosphorylation in diseased cardiomyocytes. This PhD study shows that this mechanism does not only occur in end 
stage failing hearts but also in hypertrophy with decreased cardiac function after TAC surgery. However, in the TAC model used for this PhD thesis, whole-cell PDE activities measured by a classical in vitro activity assay as well as PDE4D and PDE2 protein levels were not yet decreased, suggesting that at the investigated time point of disease progression, local microdomain-specific rather than whole-cell changes in PDE activity and possibly also localisation (Lehnart et al., 2005) might take place (Figure 31). It is noteworthy that PDE2 hydrolytic activity is stimulated by cGMP (Francis et al., 2011) suggesting an important function of this negative crosstalk mechanism within the SERCA2a microdomain during cardiac disease.

Interestingly, differences in $\beta_{1}$-AR stimulation between the bulk cytosol (Epac1-camps sensor) and the SERCA2a microdomain (Epac1-PLN sensor) were abolished in hypertrophied cardiomyocytes (Figure $29 \mathrm{G}$ ), similar to cardiomyocytes pre-treated with PDE inhibitors (Figure 25). Together with the observed alterations in PDE contribution to CAMP degradation in hypertrophied cardiomyocytes, these results reinforce the hypothesis that the privileged communication between the $\beta_{1}-A R$ and the SERCA2a microdomain is mediated by PDE dependent channelling of cAMP from the receptors to this subcellular compartment. This argues for the fact that the remodelling of PDE in various microdomains affects CAMP compartmentation in cardiac hypertrophy.

\subsubsection{Under basal conditions, PDE4 contribution to cAMP degradation is decreased in the SERCA2a compartment after TAC surgery.}

Under basal conditions, no significant differences in PDE contribution to cAMP degradation were detected in the bulk cytosol (Epac1-camps sensor) of TAC cardiomyocytes (Figure 30 D). In sharp contrast to this, basal PDE4 inhibition in cardiomyocytes of Epac1-PLN TAC mice led to a significant decrease in FRET change. This indicates a decreased basal PDE4 contribution to CAMP hydrolysis in the SERCA2a compartment of hypertrophied cardiomyocytes. However, this does not result in a decreased PLN function, as shown by $\mathrm{Ca}^{2+}$ transient measurements. Instead, the increased time of $\mathrm{Ca}^{2+}$ decay in TAC cardiomyocytes indicates a decrease in SERCA2a function associated by a tendency of decreased SERCA2a expression (Figure 32 A). As decreased PDE4 expression would increase local cAMP concentration and PLN phosphorylation, these results point toward the possibility that the local PDE4 downregulation represents a protective mechanism to initially compensate for the loss of SERCA2a function. However, the hearts of the TAC model used in this $\mathrm{PhD}$ study seem to be already transitioning towards a functionally decompensated state where the decrease of PDE4 activity can no longer compensate for the loss of SERCA2a, thereby increasing time of $\mathrm{Ca}^{2+}$ decay. In addition, a possible loss of local PKA 
activity in the SERCA2a microdomain (Zakhary et al., 2000b) might derogate the regulatory feedback loops associated with PKA-dependent PDE4 phosphorylation. A previous study showed the association of PDE4D with the SERCA2a/ PLN complex (Beca et al., 2011) and studies in human failing heart tissues revealed decreased PDE4D activity resulting from reduced expression levels (Richter et al., 2011). In contrast to this, PDE4D activity in rats with compensated hypertrophy was shown to be not significantly altered compared to sham animals (Abi-Gerges et al., 2009). In the TAC model used during this PhD thesis, PDE4D protein levels were not significantly decreased in cardiomyocytes from TAC compared to sham animals (Figure $31 \mathrm{~B}, \mathrm{C}$ ). This might indicate that in hypertrophied mouse hearts PDE4D activity, rather than PDE4D expression, is decreased. However, PDE activity assay in sham vs. TAC treated WT mice did not show any significant differences in the activity of any PDE family (Figure $31 \mathrm{~A}$ ). It is important to mention that in this case, PDE activity has been performed using a global PDE4 inhibitor. Therefore, PDE4D isoform specific inhibition would be of great advantage for following studies regarding PDE activity assays and FRET dependent CAMP measurements under real time conditions in diseased cardiomyocytes. However, as overall PDE activity does not seem to be altered in the TAC model used during this PhD thesis, one might hypothesise that PDE4 and PDE2 could be relocated during progression of hypertrophy within the cardiomyocytes to different microdomains. This would lead to altered regulation of cAMP dynamics in different subcellular locations.

\subsection{Calcium fluctuations do not influence FRET measurements via Epac1-camps}

Previously, using a classical biochemical assay and a rapid freezing technique, it has been shown that cAMP levels can rapidly change during each contraction cycle of the rat heart (Wikman-Coffelt et al., 1983). In this PhD thesis, it was demonstrated that such rapid changes do not translate into sustained changes of cytosolic AC activities under CAMPstimulating conditions in isolated mouse ventricular cardiomyocytes detected by FRET (Figure 33 and 34). A possible reason for this might be that the $\mathrm{Ca}^{2+}$ changes in field stimulated cardiomyocytes are too small and too rapid to affect the AC activities and therefore, sustained changes in cytosolic cAMP levels. However, it cannot be excluded that the rapid $\mathrm{Ca}^{2+}$ fluctuations indeed influence $\mathrm{AC}$ activity and cAMP levels in the cytosol but that the Epac1-camps biosensor might be not sensitive enough to sense these rapid fluctuations. Anyway, when cardiomyocytes are treated with the direct AC activator forskolin, this leads to a significant increase of PDE1 contribution to total PDE inhibition in field stimulated, thapsigargin or calcium ionophor treated vs. resting cells (Figure $34 \mathrm{D}$ ). This might be due to stronger or less compartmentalized CAMP elevation upon direct AC activation compared to $\beta$-adrenergic stimulation, unmasking the elevated PDE1 activity due 
to increased $\mathrm{Ca}^{2+}$ flux (Conti and Beavo, 2007). However, direct AC activation does not represent physiological conditions and is therefore usually not used in FRET experiments investigating PDE contributions to cAMP hydrolysis. As discussed elaborately in this $\mathrm{PhD}$ thesis, cAMP signalling in cardiomyocytes is known to be compartmentalized (Fischmeister et al., 2006; Zaccolo, 2009). Therefore, in the future it would be exciting to investigate whether $\mathrm{Ca}^{2+}$-induced local changes in $\mathrm{AC} 5 / 6$ activity at the plasma membrane might also propagate into subcellular compartments deep within the cell. At least measurements with the novel Epac1-PLN sensor did not show such alterations between resting and paced cardiomyocytes (Figure 23). This sensor construct contains the cytosolic Epac1-camps sensor fused to PLN, supposing the same sensor associated limitations as for the measurements in the bulk cytosol. Therefore, to really understand the $\mathrm{Ca}^{2+}$-cAMP crosstalk, one might think about the generation of CAMP sensors more sensitive to rapid changes in $\mathrm{Ca}^{2+}$ concentrations. However, in this $\mathrm{PhD}$ thesis it was important to exclude possible tampering of crucial FRET results simply due to the fact of using resting instead of field stimulated cardiomyocytes. Indeed, it has been shown that, at least when using the Epac1camps based FRET sensors, resting cardiomyocytes reflect the same condition as paced cardiomyocytes.

\subsection{Summary and Conclusions}

A novel TG mouse model expressing the localised FRET based cAMP biosensor Epac1-PLN has been successfully developed during this PhD project. Biocompatibility of the Epac1-PLN sensor has been proved, making this mouse a valuable tool to directly analyse real-time cAMP dynamics around the SERCA2a compartment in adult TG mouse cardiomyocytes with high temporal and spatial resolution. One advantage of this TG mouse line is that freshly isolated cardiomyocytes can be directly used for experiments and therefore, experimental artefacts due to virus transduction of cardiomyocytes and subsequent cultivation can be excluded. Besides, it allows the analysis of altered cAMP dynamics in in vivo models of heart disease such as hypertrophy. Using this novel Epac1-PLN sensor mouse, it has been demonstrated that the SERCA2a compartment is differentially regulated and confined from the bulk cytosol via several PDE families and their different contributions to cAMP hydrolysis. This PDE pattern changed dramatically during progression of hypertrophy, thereby affecting the privileged $\beta_{1}$-receptor-microdomain communication which here has been identified as a mechanism of cAMP compartmentation (Figure 35).

Therefore, the Epac1-PLN mouse model offers exciting new possibilities to study microdomain-specific signalling in a physiologically interesting setting of healthy and diseased organism. In the future, crossing the Epac1-PLN mouse with different disease 
models or PDE deficient mice would be of great advantage to get a more specific insight into the molecular mechanisms behind cAMP compartmentation and its alterations during progression of cardiac disease.

A

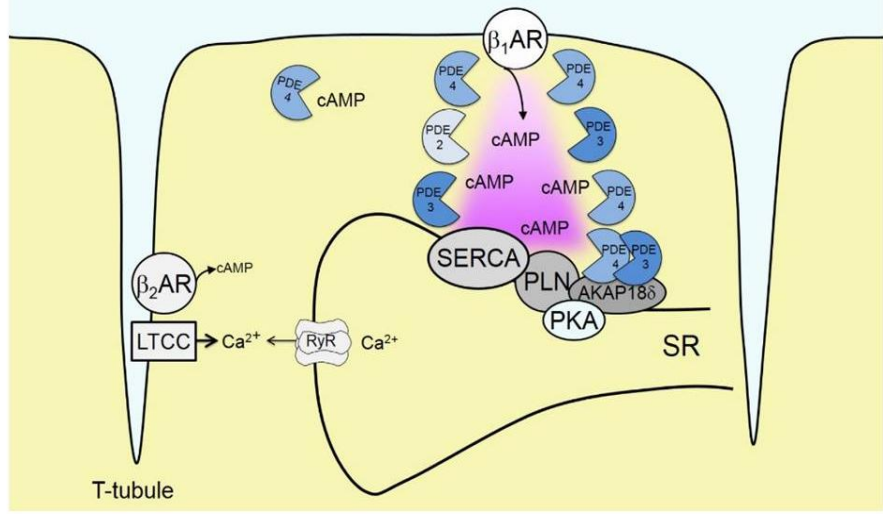

B

Hypertrophy

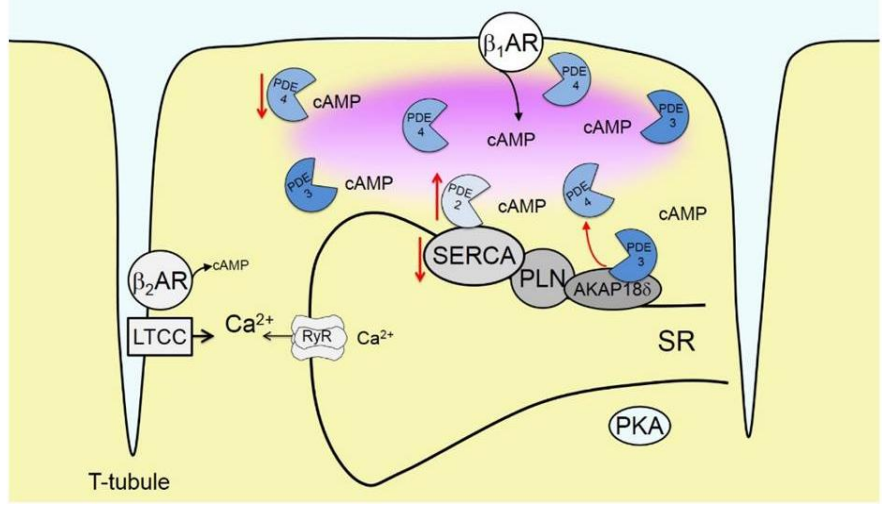

Figure $35 . \quad$ Schematics showing proposed changes in local CAMP signalling occurring in cardiac hypertrophy.

(A) In healthy cardiomyocytes, $\beta_{1}$ - but not $\beta_{2}-A R$-cAMP pools reach the SERCA2a microdomain, whereby there is a privileged communication between $\beta_{1}-A R$ and the microdomains through an imaginary "channel" formed by the orchestrated action of PDEs. The access of the cytosolic cAMP to SERCA2a in absence of receptor stimulation is controlled by the local pools of PDE3 and PDE4.

(B) In hypertrophy, the local PDE4 effect at SERCA2a is lost, while PDE2-dependent effects are increased, especially under $\beta$-AR stimulation. This remodelling of cellular PDEs leads to a loss of effective receptor-microdomain communication.

In a side project, a comparative analysis between resting and field stimulated Epac1-camps TG cardiomyocytes has been performed. It showed that rapid changes of cytosolic $\mathrm{Ca}^{2+}$ during contraction and $\beta-A R$ stimulation do not translate into sustained changes of intracellular cAMP, at least not detectable by the Epac1-camps FRET biosensor. Therefore, measurements of cAMP using Epac1-camps in resting cardiomyocytes truly represent the dynamics of this second messenger under physiologically relevant conditions. 


\section{Bibliography}

Abi-Gerges, A., Richter, W., Lefebvre, F., Mateo, P., Varin, A., Heymes, C., Samuel, J.L., Lugnier, C., Conti, M., Fischmeister, R., et al. (2009). Decreased expression and activity of cAMP phosphodiesterases in cardiac hypertrophy and its impact on beta-adrenergic cAMP signals. Circ Res 105, 784-792.

Adams, S.R., Harootunian, A.T., Buechler, Y.J., Taylor, S.S., and Tsien, R.Y. (1991). Fluorescence ratio imaging of cyclic AMP in single cells. Nature 349, 694-697.

Allen, M.D., and Zhang, J. (2006). Subcellular dynamics of protein kinase A activity visualized by FRETbased reporters. Biochem Biophys Res Commun 348, 716-721.

Altarejos, J.Y., and Montminy, M. (2011). CREB and the CRTC co-activators: sensors for hormonal and metabolic signals. Nature Reviews Molecular Cell Biology 12, 141-151.

Anger, M., Samuel, J.L., Marotte, F., Wuytack, F., Rappaport, L., and Lompre, A.M. (1994). In situ mRNA distribution of sarco(endo)plasmic reticulum $\mathrm{Ca}(2+)$-ATPase isoforms during ontogeny in the rat. J Mol Cell Cardiol 26, 539-550.

Arai, M. (2000). Function and regulation of sarcoplasmic reticulum Ca2+-ATPase: advances during the past decade and prospects for the coming decade. Jpn Heart J 41, 1-13.

Arai, M., Alpert, N.R., MacLennan, D.H., Barton, P., and Periasamy, M. (1993). Alterations in sarcoplasmic reticulum gene expression in human heart failure. A possible mechanism for alterations in systolic and diastolic properties of the failing myocardium. Circ Res 72, 463-469.

Arvanitis, D.A., Vafiadaki, E., Fan, G.C., Mitton, B.A., Gregory, K.N., Del Monte, F., KontrogianniKonstantopoulos, A., Sanoudou, D., and Kranias, E.G. (2007). Histidine-rich Ca-binding protein interacts with sarcoplasmic reticulum Ca-ATPase. Am J Physiol Heart Circ Physiol 293, H1581-1589.

Asahi, M., Nakayama, H., Tada, M., and Otsu, K. (2003). Regulation of sarco(endo)plasmic reticulum $\mathrm{Ca} 2+$ adenosine triphosphatase by phospholamban and sarcolipin: implication for cardiac hypertrophy and failure. Trends Cardiovasc Med 13, 152-157.

Babu, G.J., Bhupathy, P., Carnes, C.A., Billman, G.E., and Periasamy, M. (2007). Differential expression of sarcolipin protein during muscle development and cardiac pathophysiology. J Mol Cell Cardiol 43, 215-222.

Baillie, G.S., Sood, A., McPhee, I., Gall, I., Perry, S.J., Lefkowitz, R.J., and Houslay, M.D. (2003). betaArrestin-mediated PDE4 cAMP phosphodiesterase recruitment regulates beta-adrenoceptor switching from Gs to Gi. Proc Natl Acad Sci U S A 100, 940-945.

Barki-Harrington, L., Perrino, C., and Rockman, H.A. (2004). Network integration of the adrenergic system in cardiac hypertrophy. Cardiovasc Res 63, 391-402.

Beca, S., Ahmad, F., Shen, W., Liu, J., Makary, S., Polidovitch, N., Sun, J., Hockman, S., Chung, Y.W., Movsesian, M., et al. (2013). Phosphodiesterase type 3A regulates basal myocardial contractility through interacting with sarcoplasmic reticulum calcium ATPase type 2a signaling complexes in mouse heart. Circ Res 112, 289-297.

Beca, S., Helli, P.B., Simpson, J.A., Zhao, D., Farman, G.P., Jones, P.P., Tian, X., Wilson, L.S., Ahmad, F., Chen, S.R., et al. (2011). Phosphodiesterase 4D regulates baseline sarcoplasmic reticulum Ca2+ release and cardiac contractility, independently of L-type Ca2+ current. Circ Res 109, 1024-1030.

Bernardo, B.C., Weeks, K.L., Pretorius, L., and McMullen, J.R. (2010). Molecular distinction between physiological and pathological cardiac hypertrophy: experimental findings and therapeutic strategies. Pharmacol Ther 128, 191-227.

Bernstein, D., Fajardo, G., Zhao, M., Urashima, T., Powers, J., Berry, G., and Kobilka, B.K. (2005). Differential cardioprotective/cardiotoxic effects mediated by beta-adrenergic receptor subtypes. Am J Physiol Heart Circ Physiol 289, H2441-2449.

Bers, D.M. (2002). Cardiac excitation-contraction coupling. Nature 415, 198-205.

Biel, M., Zong, X., Ludwig, A., Sautter, A., and Hofmann, F. (1999). Structure and function of cyclic nucleotide-gated channels. Rev Physiol Biochem Pharmacol 135, 151-171. 
Bodor, J., Bopp, T., Vaeth, M., Klein, M., Serfling, E., Hunig, T., Becker, C., Schild, H., and Schmitt, E. (2012). Cyclic AMP underpins suppression by regulatory T cells. Eur J Immunol 42, 1375-1384.

Boknik, P., Fockenbrock, M., Herzig, S., Knapp, J., Linck, B., Luss, H., Muller, F.U., Muller, T., Schmitz, W., Schroder, F., et al. (2000). Protein phosphatase activity is increased in a rat model of long-term beta-adrenergic stimulation. Naunyn Schmiedebergs Arch Pharmacol 362, 222-231.

Borner, S., Schwede, F., Schlipp, A., Berisha, F., Calebiro, D., Lohse, M.J., and Nikolaev, V.O. (2011). FRET measurements of intracellular CAMP concentrations and CAMP analog permeability in intact cells. Nat Protoc 6, 427-438.

Brilla, C.G., Pick, R., Tan, L.B., Janicki, J.S., and Weber, K.T. (1990). Remodeling of the rat right and left ventricles in experimental hypertension. Circ Res 67, 1355-1364.

Bristow, M.R., Ginsburg, R., Minobe, W., Cubicciotti, R.S., Sageman, W.S., Lurie, K., Billingham, M.E., Harrison, D.C., and Stinson, E.B. (1982). Decreased catecholamine sensitivity and beta-adrenergicreceptor density in failing human hearts. N Engl J Med 307, 205-211.

Brittsan, A.G., Ginsburg, K.S., Chu, G., Yatani, A., Wolska, B.M., Schmidt, A.G., Asahi, M., MacLennan, D.H., Bers, D.M., and Kranias, E.G. (2003). Chronic SR Ca2+-ATPase inhibition causes adaptive changes in cellular Ca2+ transport. Circ Res 92, 769-776.

Brooker, G., Harper, J.F., Terasaki, W.L., and Moylan, R.D. (1979). Radioimmunoassay of cyclic AMP and cyclic GMP. Adv Cyclic Nucleotide Res 10, 1-33.

Brown, K.M., Lee, L.C., Findlay, J.E., Day, J.P., and Baillie, G.S. (2012). Cyclic AMP-specific phosphodiesterase, PDE8A1, is activated by protein kinase A-mediated phosphorylation. FEBS Lett 586, 1631-1637.

Brudvik, K.W., and Tasken, K. (2012). Modulation of T cell immune functions by the prostaglandin E(2) - cAMP pathway in chronic inflammatory states. Br J Pharmacol 166, 411-419.

Brunton, L.L., Hayes, J.S., and Mayer, S.E. (1979). Hormonally specific phosphorylation of cardiac troponin I and activation of glycogen phosphorylase. Nature 280, 78-80.

Brunton, L.L., Hayes, J.S., and Mayer, S.E. (1981). Functional compartmentation of cyclic AMP and protein kinase in heart. Adv Cyclic Nucleotide Res 14, 391-397.

Buitrago, M., Lorenz, K., Maass, A.H., Oberdorf-Maass, S., Keller, U., Schmitteckert, E.M., Ivashchenko, Y., Lohse, M.J., and Engelhardt, S. (2005). The transcriptional repressor Nab1 is a specific regulator of pathological cardiac hypertrophy. Nat Med 11, 837-844.

Buxton, I.L., and Brunton, L.L. (1983). Compartments of cyclic AMP and protein kinase in mammalian cardiomyocytes. J Biol Chem 258, 10233-10239.

Calebiro, D., Nikolaev, V.O., Gagliani, M.C., de Filippis, T., Dees, C., Tacchetti, C., Persani, L., and Lohse, M.J. (2009). Persistent cAMP-signals triggered by internalized G-protein-coupled receptors. PLoS Biol 7, e1000172.

Chen, J., Levin, L.R., and Buck, J. (2012). Role of soluble adenylyl cyclase in the heart. Am J Physiol Heart Circ Physiol 302, H538-543.

Chen, Z., Akin, B.L., and Jones, L.R. (2010). Ca2+ binding to site I of the cardiac Ca2+ pump is sufficient to dissociate phospholamban. J Biol Chem 285, 3253-3260.

Chu, G., Egnaczyk, G.F., Zhao, W., Jo, S.H., Fan, G.C., Maggio, J.E., Xiao, R.P., and Kranias, E.G. (2004). Phosphoproteome analysis of cardiomyocytes subjected to beta-adrenergic stimulation: identification and characterization of a cardiac heat shock protein p20. Circ Res 94, 184-193.

Chu, G., Lester, J.W., Young, K.B., Luo, W., Zhai, J., and Kranias, E.G. (2000). A single site (Ser16) phosphorylation in phospholamban is sufficient in mediating its maximal cardiac responses to beta agonists. J Biol Chem 275, 38938-38943.

Colucci, W.S. (1991). Cardiovascular effects of milrinone. Am Heart J 121, 1945-1947.

Communal, C., Singh, K., Sawyer, D.B., and Colucci, W.S. (1999). Opposing effects of beta(1)- and beta(2)-adrenergic receptors on cardiac myocyte apoptosis : role of a pertussis toxin-sensitive $G$ protein. Circulation 100, 2210-2212.

Conti, M., and Beavo, J. (2007). Biochemistry and physiology of cyclic nucleotide phosphodiesterases: essential components in cyclic nucleotide signaling. Annu Rev Biochem 76, 481-511.

Cooper, P.D. (1961). The plaque assay of animal viruses. Adv Virus Res 8, 319-378. 
Corbin, J.D., Sugden, P.H., Lincoln, T.M., and Keely, S.L. (1977). Compartmentalization of adenosine $3^{\prime}: 5^{\prime}$-monophosphate and adenosine 3':5'-monophosphate-dependent protein kinase in heart tissue. J Biol Chem 252, 3854-3861.

Cornea, R.L., Jones, L.R., Autry, J.M., and Thomas, D.D. (1997). Mutation and phosphorylation change the oligomeric structure of phospholamban in lipid bilayers. Biochemistry 36, 2960-2967.

Craven, K.B., and Zagotta, W.N. (2006). CNG and HCN channels: two peas, one pod. Annu Rev Physiol 68, 375-401.

De Arcangelis, V., Liu, S., Zhang, D., Soto, D., and Xiang, Y.K. (2010). Equilibrium between adenylyl cyclase and phosphodiesterase patterns adrenergic agonist dose-dependent spatiotemporal cAMP/protein kinase A activities in cardiomyocytes. Mol Pharmacol 78, 340-349.

Depry, C., Allen, M.D., and Zhang, J. (2011). Visualization of PKA activity in plasma membrane microdomains. Mol Biosyst 7, 52-58.

Despa, S., Bossuyt, J., Han, F., Ginsburg, K.S., Jia, L.G., Kutchai, H., Tucker, A.L., and Bers, D.M. (2005). Phospholemman-phosphorylation mediates the beta-adrenergic effects on $\mathrm{Na} / \mathrm{K}$ pump function in cardiac myocytes. Circ Res 97, 252-259.

Devereux, R.B., Pickering, T.G., Alderman, M.H., Chien, S., Borer, J.S., and Laragh, J.H. (1987). Left ventricular hypertrophy in hypertension. Prevalence and relationship to pathophysiologic variables. Hypertension 9, II53-60.

Di Benedetto, G., Zoccarato, A., Lissandron, V., Terrin, A., Li, X., Houslay, M.D., Baillie, G.S., and Zaccolo, M. (2008). Protein kinase A type I and type II define distinct intracellular signaling compartments. Circ Res 103, 836-844.

Diller, T.C., Madhusudan, Xuong, N.H., and Taylor, S.S. (2001). Molecular basis for regulatory subunit diversity in CAMP-dependent protein kinase: crystal structure of the type II beta regulatory subunit. Structure 9, 73-82.

DiPilato, L.M., Cheng, X., and Zhang, J. (2004). Fluorescent indicators of cAMP and Epac activation reveal differential dynamics of cAMP signaling within discrete subcellular compartments. Proc Natl Acad Sci U S A 101, 16513-16518.

Diviani, D., Dodge-Kafka, K.L., Li, J., and Kapiloff, M.S. (2011). A-kinase anchoring proteins: scaffolding proteins in the heart. Am J Physiol Heart Circ Physiol 301, H1742-1753.

Diwan, A., and Dorn, G.W., 2nd (2007). Decompensation of cardiac hypertrophy: cellular mechanisms and novel therapeutic targets. Physiology (Bethesda) 22, 56-64.

Dodge, K.L., Khouangsathiene, S., Kapiloff, M.S., Mouton, R., Hill, E.V., Houslay, M.D., Langeberg, L.K., and Scott, J.D. (2001). mAKAP assembles a protein kinase A/PDE4 phosphodiesterase cAMP signaling module. Embo J 20, 1921-1930.

Dong, X., and Thomas, D.D. (2014). Time-resolved FRET Reveals the Structural Mechanism of SERCAPLB Regulation. Biochem Biophys Res Commun.

El-Armouche, A., Gocht, F., Jaeckel, E., Wittkopper, K., Peeck, M., and Eschenhagen, T. (2007). Longterm beta-adrenergic stimulation leads to downregulation of protein phosphatase inhibitor-1 in the heart. Eur J Heart Fail 9, 1077-1080.

El-Armouche, A., Pamminger, T., Ditz, D., Zolk, O., and Eschenhagen, T. (2004). Decreased protein and phosphorylation level of the protein phosphatase inhibitor-1 in failing human hearts. Cardiovasc Res 61, 87-93.

El-Armouche, A., Rau, T., Zolk, O., Ditz, D., Pamminger, T., Zimmermann, W.H., Jackel, E., Harding, S.E., Boknik, P., Neumann, J., et al. (2003). Evidence for protein phosphatase inhibitor-1 playing an amplifier role in beta-adrenergic signaling in cardiac myocytes. FASEB J 17, 437-439.

Fabiato, A., and Fabiato, F. (1977). Calcium release from the sarcoplasmic reticulum. Circ Res 40, 119129.

Fan, G.C., Chu, G., and Kranias, E.G. (2005). Hsp20 and its cardioprotection. Trends Cardiovasc Med $15,138-141$.

Fan, G.C., Yuan, Q., Song, G., Wang, Y., Chen, G., Qian, J., Zhou, X., Lee, Y.J., Ashraf, M., and Kranias, E.G. (2006). Small heat-shock protein Hsp2O attenuates beta-agonist-mediated cardiac remodeling through apoptosis signal-regulating kinase 1 . Circ Res 99, 1233-1242. 
Ferguson, S.S. (2001). Evolving concepts in G protein-coupled receptor endocytosis: the role in receptor desensitization and signaling. Pharmacol Rev 53, 1-24.

Fischmeister, R., Castro, L.R., Abi-Gerges, A., Rochais, F., Jurevicius, J., Leroy, J., and Vandecasteele, G. (2006). Compartmentation of cyclic nucleotide signaling in the heart: the role of cyclic nucleotide phosphodiesterases. Circ Res 99, 816-828.

Förster, T. (1948). Zwischenmolekulare energiewanderung und fluoreszenz. Ann Physik 437, 55-75.

Francis, S.H., Blount, M.A., and Corbin, J.D. (2011). Mammalian cyclic nucleotide phosphodiesterases: molecular mechanisms and physiological functions. Physiol Rev 91, 651-690.

Frantz, S., Klaiber, M., Baba, H.A., Oberwinkler, H., Volker, K., Gabetaner, B., Bayer, B., Abebetaer, M., Schuh, K., Feil, R., et al. (2013). Stress-dependent dilated cardiomyopathy in mice with cardiomyocyte-restricted inactivation of cyclic GMP-dependent protein kinase I. Eur Heart J 34, 12331244.

Frings, S., Seifert, R., Godde, M., and Kaupp, U.B. (1995). Profoundly different calcium permeation and blockage determine the specific function of distinct cyclic nucleotide-gated channels. Neuron 15, 169-179.

Fuller, W., Eaton, P., Bell, J.R., and Shattock, M.J. (2004). Ischemia-induced phosphorylation of phospholemman directly activates rat cardiac Na/K-ATPase. FASEB J 18, 197-199.

Ganau, A., Devereux, R.B., Roman, M.J., de Simone, G., Pickering, T.G., Saba, P.S., Vargiu, P., Simongini, I., and Laragh, J.H. (1992). Patterns of left ventricular hypertrophy and geometric remodeling in essential hypertension. J Am Coll Cardiol 19, 1550-1558.

Gao, T., Yatani, A., Dell'Acqua, M.L., Sako, H., Green, S.A., Dascal, N., Scott, J.D., and Hosey, M.M. (1997). cAMP-dependent regulation of cardiac L-type Ca2+ channels requires membrane targeting of PKA and phosphorylation of channel subunits. Neuron 19, 185-196.

Ghigo, A., Perino, A., Mehel, H., Zahradnikova, A., Jr., Morello, F., Leroy, J., Nikolaev, V.O., Damilano, F., Cimino, J., De Luca, E., et al. (2012). Phosphoinositide 3-kinase gamma protects against catecholamine-induced ventricular arrhythmia through protein kinase A-mediated regulation of distinct phosphodiesterases. Circulation 126, 2073-2083.

Gloerich, M., and Bos, J.L. (2010). Epac: defining a new mechanism for cAMP action. Annu Rev Pharmacol Toxicol 50, 355-375.

Gregory, K.N., Ginsburg, K.S., Bodi, I., Hahn, H., Marreez, Y.M., Song, Q., Padmanabhan, P.A., Mitton, B.A., Waggoner, J.R., Del Monte, F., et al. (2006). Histidine-rich Ca binding protein: a regulator of sarcoplasmic reticulum calcium sequestration and cardiac function. J Mol Cell Cardiol 40, 653-665.

Gupta, R.C., Mishra, S., Rastogi, S., Imai, M., Habib, O., and Sabbah, H.N. (2003). Cardiac SR-coupled PP1 activity and expression are increased and inhibitor 1 protein expression is decreased in failing hearts. Am J Physiol Heart Circ Physiol 285, H2373-2381.

Gupta, R.C., Mishra, S., Yang, X.P., and Sabbah, H.N. (2005). Reduced inhibitor 1 and 2 activity is associated with increased protein phosphatase type 1 activity in left ventricular myocardium of onekidney, one-clip hypertensive rats. Mol Cell Biochem 269, 49-57.

Gustavsson, M., Verardi, R., Mullen, D.G., Mote, K.R., Traaseth, N.J., Gopinath, T., and Veglia, G. (2013). Allosteric regulation of SERCA by phosphorylation-mediated conformational shift of phospholamban. Proc Natl Acad Sci U S A 110, 17338-17343.

Han, Y., Chen, Y.S., Liu, Z., Bodyak, N., Rigor, D., Bisping, E., Pu, W.T., and Kang, P.M. (2006). Overexpression of HAX-1 protects cardiac myocytes from apoptosis through caspase-9 inhibition. Circ Res 99, 415-423.

Harper, J.F., and Brooker, G. (1975). Femtomole sensitive radioimmunoassay for cyclic AMP and cyclic GMP after 2'0 acetylation by acetic anhydride in aqueous solution. J Cyclic Nucleotide Res 1, 207-218.

Hasenfuss, G., Reinecke, H., Studer, R., Meyer, M., Pieske, B., Holtz, J., Holubarsch, C., Posival, H., Just, H., and Drexler, H. (1994). Relation between myocardial function and expression of sarcoplasmic reticulum $\mathrm{Ca}(2+)$-ATPase in failing and nonfailing human myocardium. Circ Res 75, 434-442.

Hayes, J.S., Brunton, L.L., Brown, J.H., Reese, J.B., and Mayer, S.E. (1979). Hormonally specific expression of cardiac protein kinase activity. Proc Natl Acad Sci U S A 76, 1570-1574. 
Hein, P., Rochais, F., Hoffmann, C., Dorsch, S., Nikolaev, V.O., Engelhardt, S., Berlot, C.H., Lohse, M.J., and Bunemann, M. (2006). Gs activation is time-limiting in initiating receptor-mediated signaling. J Biol Chem 281, 33345-33351.

Hellstern, S., Pegoraro, S., Karim, C.B., Lustig, A., Thomas, D.D., Moroder, L., and Engel, J. (2001). Sarcolipin, the shorter homologue of phospholamban, forms oligomeric structures in detergent micelles and in liposomes. J Biol Chem 276, 30845-30852.

Herget, S., Lohse, M.J., and Nikolaev, V.O. (2008). Real-time monitoring of phosphodiesterase inhibition in intact cells. Cell Signal 20,1423-1431.

Holz, G.G. (2004). Epac: A new cAMP-binding protein in support of glucagon-like peptide-1 receptormediated signal transduction in the pancreatic beta-cell. Diabetes 53, 5-13.

Horner, A., Goetz, F., Tampe, R., Klussmann, E., and Pohl, P. (2012). Mechanism for targeting the Akinase anchoring protein AKAP18delta to the membrane. J Biol Chem 287, 42495-42501.

Houser, S.R., Piacentino, V., 3rd, and Weisser, J. (2000). Abnormalities of calcium cycling in the hypertrophied and failing heart. J Mol Cell Cardiol 32, 1595-1607.

Houslay, M.D., and Adams, D.R. (2003). PDE4 cAMP phosphodiesterases: modular enzymes that orchestrate signalling cross-talk, desensitization and compartmentalization. Biochem J 370, 1-18.

Houslay, M.D., Baillie, G.S., and Maurice, D.H. (2007). cAMP-Specific phosphodiesterase-4 enzymes in the cardiovascular system: a molecular toolbox for generating compartmentalized cAMP signaling. Circ Res 100, 950-966.

Houslay, M.D., Schafer, P., and Zhang, K.Y. (2005). Keynote review: phosphodiesterase-4 as a therapeutic target. Drug Discov Today 10, 1503-1519.

Hu, P., Zhang, D., Swenson, L., Chakrabarti, G., Abel, E.D., and Litwin, S.E. (2003). Minimally invasive aortic banding in mice: effects of altered cardiomyocyte insulin signaling during pressure overload. Am J Physiol Heart Circ Physiol 285, H1261-1269.

Huang, B., Wang, S., Qin, D., Boutjdir, M., and El-Sherif, N. (1999). Diminished basal phosphorylation level of phospholamban in the postinfarction remodeled rat ventricle: role of beta-adrenergic pathway, G(i) protein, phosphodiesterase, and phosphatases. Circ Res 85, 848-855.

Huang, F., Shan, J., Reiken, S., Wehrens, X.H., and Marks, A.R. (2006). Analysis of calstabin2 (FKBP12.6)-ryanodine receptor interactions: rescue of heart failure by calstabin2 in mice. Proc Natl Acad Sci U S A 103, 3456-3461.

Hulme, J.T., Lin, T.W., Westenbroek, R.E., Scheuer, T., and Catterall, W.A. (2003). Beta-adrenergic regulation requires direct anchoring of PKA to cardiac CaV1.2 channels via a leucine zipper interaction with A kinase-anchoring protein 15. Proc Natl Acad Sci U S A 100, 13093-13098.

lancu, R.V., Ramamurthy, G., Warrier, S., Nikolaev, V.O., Lohse, M.J., Jones, S.W., and Harvey, R.D. (2008). Cytoplasmic cAMP concentrations in intact cardiac myocytes. Am J Physiol Cell Physiol 295, C414-422.

Inui, M., Chamberlain, B.K., Saito, A., and Fleischer, S. (1986). The nature of the modulation of Ca2+ transport as studied by reconstitution of cardiac sarcoplasmic reticulum. J Biol Chem 261, 1794-1800. James, P., Inui, M., Tada, M., Chiesi, M., and Carafoli, E. (1989). Nature and site of phospholamban regulation of the Ca2+ pump of sarcoplasmic reticulum. Nature 342, 90-92.

Jaski, B.E., Jessup, M.L., Mancini, D.M., Cappola, T.P., Pauly, D.F., Greenberg, B., Borow, K., Dittrich, H., Zsebo, K.M., and Hajjar, R.J. (2009). Calcium upregulation by percutaneous administration of gene therapy in cardiac disease (CUPID Trial), a first-in-human phase $1 / 2$ clinical trial. J Card Fail 15, 171181.

Jessup, M., Greenberg, B., Mancini, D., Cappola, T., Pauly, D.F., Jaski, B., Yaroshinsky, A., Zsebo, K.M., Dittrich, H., and Hajjar, R.J. (2011). Calcium Upregulation by Percutaneous Administration of Gene Therapy in Cardiac Disease (CUPID): a phase 2 trial of intracoronary gene therapy of sarcoplasmic reticulum Ca2+-ATPase in patients with advanced heart failure. Circulation 124, 304-313.

Jurevicius, J., and Fischmeister, R. (1996). cAMP compartmentation is responsible for a local activation of cardiac Ca2+ channels by beta-adrenergic agonists. Proc Natl Acad Sci U S A 93, 295-299. 
Kadambi, V.J., Ponniah, S., Harrer, J.M., Hoit, B.D., Dorn, G.W., 2nd, Walsh, R.A., and Kranias, E.G. (1996). Cardiac-specific overexpression of phospholamban alters calcium kinetics and resultant cardiomyocyte mechanics in transgenic mice. J Clin Invest 97, 533-539.

Kandel, E.R. (2001). The molecular biology of memory storage: a dialogue between genes and synapses. Science 294, 1030-1038.

Kawasaki, H., Springett, G.M., Mochizuki, N., Toki, S., Nakaya, M., Matsuda, M., Housman, D.E., and Graybiel, A.M. (1998). A family of cAMP-binding proteins that directly activate Rap1. Science 282, 2275-2279.

Keef, K.D., Hume, J.R., and Zhong, J. (2001). Regulation of cardiac and smooth muscle $\mathrm{Ca}(2+)$ channels (Ca(V)1.2a,b) by protein kinases. Am J Physiol Cell Physiol 281, C1743-1756.

Kerfant, B.G., Zhao, D., Lorenzen-Schmidt, I., Wilson, L.S., Cai, S., Chen, S.R., Maurice, D.H., and Backx, P.H. (2007). PI3Kgamma is required for PDE4, not PDE3, activity in subcellular microdomains containing the sarcoplasmic reticular calcium ATPase in cardiomyocytes. Circ Res 101, 400-408.

Kho, C., Lee, A., Jeong, D., Oh, J.G., Chaanine, A.H., Kizana, E., Park, W.J., and Hajjar, R.J. (2011). SUMO1-dependent modulation of SERCA2a in heart failure. Nature 477, 601-605.

Kim, C., Xuong, N.H., and Taylor, S.S. (2005). Crystal structure of a complex between the catalytic and regulatory (Rlalpha) subunits of PKA. Science 307, 690-696.

Kim, H.W., Steenaart, N.A., Ferguson, D.G., and Kranias, E.G. (1990). Functional reconstitution of the cardiac sarcoplasmic reticulum Ca2(+)-ATPase with phospholamban in phospholipid vesicles. J Biol Chem 265, 1702-1709.

Kimura, Y., Kurzydlowski, K., Tada, M., and MacLennan, D.H. (1997). Phospholamban inhibitory function is activated by depolymerization. J Biol Chem 272, 15061-15064.

Kirchberger, M.A., Tada, M., Repke, D.I., and Katz, A.M. (1972). Cyclic adenosine 3',5'monophosphate-dependent protein kinase stimulation of calcium uptake by canine cardiac microsomes. J Mol Cell Cardiol 4, 673-680.

Kiriazis, H., and Kranias, E.G. (2000). Genetically engineered models with alterations in cardiac membrane calcium-handling proteins. Annu Rev Physiol 62, 321-351.

Kostic, M.M., Erdogan, S., Rena, G., Borchert, G., Hoch, B., Bartel, S., Scotland, G., Huston, E., Houslay, M.D., and Krause, E.G. (1997). Altered expression of PDE1 and PDE4 cyclic nucleotide phosphodiesterase isoforms in 7-oxo-prostacyclin-preconditioned rat heart. J Mol Cell Cardiol 29, 3135-3146.

Krebs, E.G., and Beavo, J.A. (1979). Phosphorylation-dephosphorylation of enzymes. Annu Rev Biochem 48, 923-959.

Kuschel, M., Karczewski, P., Hempel, P., Schlegel, W.P., Krause, E.G., and Bartel, S. (1999a). Ser16 prevails over Thr17 phospholamban phosphorylation in the beta-adrenergic regulation of cardiac relaxation. Am J Physiol 276, H1625-1633.

Kuschel, M., Zhou, Y.Y., Spurgeon, H.A., Bartel, S., Karczewski, P., Zhang, S.J., Krause, E.G., Lakatta, E.G., and Xiao, R.P. (1999b). beta2-adrenergic CAMP signaling is uncoupled from phosphorylation of cytoplasmic proteins in canine heart. Circulation 99, 2458-2465.

Laemmli, U.K. (1970). Cleavage of structural proteins during the assembly of the head of bacteriophage T4. Nature 227, 680-685.

Lamb, T.D., and Pugh, E.N., Jr. (1992). G-protein cascades: gain and kinetics. Trends Neurosci 15, 291298.

Leech, C.A., Chepurny, O.G., and Holz, G.G. (2010). Epac2-dependent rap1 activation and the control of islet insulin secretion by glucagon-like peptide-1. Vitam Horm 84, 279-302.

Lehnart, S.E., Wehrens, X.H., Reiken, S., Warrier, S., Belevych, A.E., Harvey, R.D., Richter, W., Jin, S.L., Conti, M., and Marks, A.R. (2005). Phosphodiesterase 4D deficiency in the ryanodine-receptor complex promotes heart failure and arrhythmias. Cell 123, 25-35.

Leroy, J., Abi-Gerges, A., Nikolaev, V.O., Richter, W., Lechene, P., Mazet, J.L., Conti, M., Fischmeister, R., and Vandecasteele, G. (2008). Spatiotemporal dynamics of beta-adrenergic cAMP signals and Ltype $\mathrm{Ca} 2+$ channel regulation in adult rat ventricular myocytes: role of phosphodiesterases. Circ Res $102,1091-1100$. 
Leroy, J., Richter, W., Mika, D., Castro, L.R., Abi-Gerges, A., Xie, M., Scheitrum, C., Lefebvre, F., Schittl, J., Mateo, P., et al. (2011). Phosphodiesterase 4B in the cardiac L-type $\mathrm{Ca}(2)(+)$ channel complex regulates $\mathrm{Ca}(2)(+)$ current and protects against ventricular arrhythmias in mice. J Clin Invest 121, 2651-2661.

Levy, D., Larson, M.G., Vasan, R.S., Kannel, W.B., and Ho, K.K. (1996). The progression from hypertension to congestive heart failure. JAMA 275, 1557-1562.

Limas, C.J., Olivari, M.T., Goldenberg, I.F., Levine, T.B., Benditt, D.G., and Simon, A. (1987). Calcium uptake by cardiac sarcoplasmic reticulum in human dilated cardiomyopathy. Cardiovasc Res 21, 601605.

Lindemann, J.P., Jones, L.R., Hathaway, D.R., Henry, B.G., and Watanabe, A.M. (1983). betaAdrenergic stimulation of phospholamban phosphorylation and Ca2+-ATPase activity in guinea pig ventricles. J Biol Chem 258, 464-471.

Lindemann, J.P., and Watanabe, A.M. (1985). Phosphorylation of phospholamban in intact myocardium. Role of Ca2+-calmodulin-dependent mechanisms. J Biol Chem 260, 4516-4525.

Liu, S., Li, Y., Kim, S., Fu, Q., Parikh, D., Sridhar, B., Shi, Q., Zhang, X., Guan, Y., Chen, X., et al. (2012). Phosphodiesterases coordinate CAMP propagation induced by two stimulatory $G$ protein-coupled receptors in hearts. Proc Natl Acad Sci U S A 109, 6578-6583.

Liu, S., Zhang, J., and Xiang, Y.K. (2011). FRET-based direct detection of dynamic protein kinase A activity on the sarcoplasmic reticulum in cardiomyocytes. Biochem Biophys Res Commun 404, 581586.

Lohse, M.J., Engelhardt, S., and Eschenhagen, T. (2003). What is the role of beta-adrenergic signaling in heart failure? Circ Res 93, 896-906.

Lompre, A.M., Anger, M., and Levitsky, D. (1994). Sarco(endo)plasmic reticulum calcium pumps in the cardiovascular system: function and gene expression. J Mol Cell Cardiol 26, 1109-1121.

Lompre, A.M., Hajjar, R.J., Harding, S.E., Kranias, E.G., Lohse, M.J., and Marks, A.R. (2010). Ca2+ cycling and new therapeutic approaches for heart failure. Circulation 121, 822-830.

Lompre, A.M., Lambert, F., Lakatta, E.G., and Schwartz, K. (1991). Expression of sarcoplasmic reticulum $\mathrm{Ca}(2+)$-ATPase and calsequestrin genes in rat heart during ontogenic development and aging. Circ Res 69, 1380-1388.

Lygren, B., Carlson, C.R., Santamaria, K., Lissandron, V., McSorley, T., Litzenberg, J., Lorenz, D., Wiesner, B., Rosenthal, W., Zaccolo, M., et al. (2007). AKAP complex regulates Ca2+ re-uptake into heart sarcoplasmic reticulum. EMBO Rep 8, 1061-1067.

Lyon, A.R., Bannister, M.L., Collins, T., Pearce, E., Sepehripour, A.H., Dubb, S.S., Garcia, E., O'Gara, P., Liang, L., Kohlbrenner, E., et al. (2011). SERCA2a gene transfer decreases sarcoplasmic reticulum calcium leak and reduces ventricular arrhythmias in a model of chronic heart failure. Circ Arrhythm Electrophysiol 4, 362-372.

MacDougall, L.K., Jones, L.R., and Cohen, P. (1991). Identification of the major protein phosphatases in mammalian cardiac muscle which dephosphorylate phospholamban. Eur J Biochem 196, 725-734.

MacKenzie, S.J., Baillie, G.S., McPhee, I., MacKenzie, C., Seamons, R., McSorley, T., Millen, J., Beard, M.B., van Heeke, G., and Houslay, M.D. (2002). Long PDE4 CAMP specific phosphodiesterases are activated by protein kinase A-mediated phosphorylation of a single serine residue in Upstream Conserved Region 1 (UCR1). Br J Pharmacol 136, 421-433.

MacLennan, D.H. (1970). Purification and properties of an adenosine triphosphatase from sarcoplasmic reticulum. J Biol Chem 245, 4508-4518.

MacLennan, D.H., and Kranias, E.G. (2003). Phospholamban: a crucial regulator of cardiac contractility. Nat Rev Mol Cell Biol 4, 566-577.

Maier, L.S., Bers, D.M., and Brown, J.H. (2007). Calmodulin and Ca2+/calmodulin kinases in the heart - physiology and pathophysiology. Cardiovasc Res 73, 629-630.

Martinez, S.E., Wu, A.Y., Glavas, N.A., Tang, X.B., Turley, S., Hol, W.G., and Beavo, J.A. (2002). The two GAF domains in phosphodiesterase $2 \mathrm{~A}$ have distinct roles in dimerization and in CGMP binding. Proc Natl Acad Sci U S A 99, 13260-13265. 
Marx, S.O., Reiken, S., Hisamatsu, Y., Jayaraman, T., Burkhoff, D., Rosemblit, N., and Marks, A.R. (2000). PKA phosphorylation dissociates FKBP12.6 from the calcium release channel (ryanodine receptor): defective regulation in failing hearts. Cell 101, 365-376.

Mattiazzi, A., Mundina-Weilenmann, C., Guoxiang, C., Vittone, L., and Kranias, E. (2005). Role of phospholamban phosphorylation on Thr17 in cardiac physiological and pathological conditions. Cardiovasc Res 68, 366-375.

Mauban, J.R., O'Donnell, M., Warrier, S., Manni, S., and Bond, M. (2009). AKAP-scaffolding proteins and regulation of cardiac physiology. Physiology (Bethesda) 24, 78-87.

Mehel, H., Emons, J., Vettel, C., Wittkopper, K., Seppelt, D., Dewenter, M., Lutz, S., Sossalla, S., Maier, L.S., Lechene, P., et al. (2013). Phosphodiesterase-2 is up-regulated in human failing hearts and blunts beta-adrenergic responses in cardiomyocytes. J Am Coll Cardiol 62, 1596-1606.

Meyer, M., Schillinger, W., Pieske, B., Holubarsch, C., Heilmann, C., Posival, H., Kuwajima, G., Mikoshiba, K., Just, H., Hasenfuss, G., et al. (1995). Alterations of sarcoplasmic reticulum proteins in failing human dilated cardiomyopathy. Circulation 92, 778-784.

Michel, M.C., Maisel, A.S., and Brodde, O.E. (1990). Mitigation of beta 1- and/or beta 2-adrenoceptor function in human heart failure. Br J Clin Pharmacol 30 Suppl 1, 37S-42S.

Miller, C.L., and Yan, C. (2010). Targeting cyclic nucleotide phosphodiesterase in the heart: therapeutic implications. J Cardiovasc Transl Res 3, 507-515.

Minamisawa, S., Wang, Y., Chen, J., Ishikawa, Y., Chien, K.R., and Matsuoka, R. (2003). Atrial chamber-specific expression of sarcolipin is regulated during development and hypertrophic remodeling. J Biol Chem 278, 9570-9575.

Mohamed, T.M., Zakeri, S.A., Baudoin, F., Wolf, M., Oceandy, D., Cartwright, E.J., Gul, S., and Neyses, L. (2013). Optimisation and validation of a high throughput screening compatible assay to identify inhibitors of the plasma membrane calcium ATPase pump--a novel therapeutic target for contraception and malaria. J Pharm Pharm Sci 16, 217-230.

Mokni, W., Keravis, T., Etienne-Selloum, N., Walter, A., Kane, M.O., Schini-Kerth, V.B., and Lugnier, C. (2010). Concerted regulation of cGMP and cAMP phosphodiesterases in early cardiac hypertrophy induced by angiotensin II. PLoS One 5, e14227.

Molina, C.E., Leroy, J., Richter, W., Xie, M., Scheitrum, C., Lee, I.O., Maack, C., Rucker-Martin, C., Donzeau-Gouge, P., Verde, I., et al. (2012). Cyclic adenosine monophosphate phosphodiesterase type 4 protects against atrial arrhythmias. J Am Coll Cardiol 59, 2182-2190.

Mongillo, M., McSorley, T., Evellin, S., Sood, A., Lissandron, V., Terrin, A., Huston, E., Hannawacker, A., Lohse, M.J., Pozzan, T., et al. (2004). Fluorescence resonance energy transfer-based analysis of CAMP dynamics in live neonatal rat cardiac myocytes reveals distinct functions of compartmentalized phosphodiesterases. Circ Res 95, 67-75.

Morozov, A., Muzzio, I.A., Bourtchouladze, R., Van-Strien, N., Lapidus, K., Yin, D., Winder, D.G., Adams, J.P., Sweatt, J.D., and Kandel, E.R. (2003). Rap1 couples CAMP signaling to a distinct pool of p42/44MAPK regulating excitability, synaptic plasticity, learning, and memory. Neuron 39, 309-325.

Movsesian, M., Stehlik, J., Vandeput, F., and Bristow, M.R. (2009). Phosphodiesterase inhibition in heart failure. Heart Fail Rev 14, 255-263.

Movsesian, M.A., Nishikawa, M., and Adelstein, R.S. (1984). Phosphorylation of phospholamban by calcium-activated, phospholipid-dependent protein kinase. Stimulation of cardiac sarcoplasmic reticulum calcium uptake. J Biol Chem 259, 8029-8032.

Mueller, B., Karim, C.B., Negrashov, I.V., Kutchai, H., and Thomas, D.D. (2004). Direct detection of phospholamban and sarcoplasmic reticulum Ca-ATPase interaction in membranes using fluorescence resonance energy transfer. Biochemistry 43, 8754-8765.

Muller, F.U., Boknik, P., Knapp, J., Linck, B., Luss, H., Neumann, J., and Schmitz, W. (2001). Activation and inactivation of cAMP-response element-mediated gene transcription in cardiac myocytes. Cardiovasc Res 52, 95-102.

Mundina-Weilenmann, C., Vittone, L., Ortale, M., de Cingolani, G.C., and Mattiazzi, A. (1996). Immunodetection of phosphorylation sites gives new insights into the mechanisms underlying phospholamban phosphorylation in the intact heart. J Biol Chem 271, 33561-33567. 
Nagai, R., Zarain-Herzberg, A., Brandl, C.J., Fujii, J., Tada, M., MacLennan, D.H., Alpert, N.R., and Periasamy, M. (1989). Regulation of myocardial Ca2+-ATPase and phospholamban mRNA expression in response to pressure overload and thyroid hormone. Proc Natl Acad Sci U S A 86, 2966-2970.

Neef, S., Dybkova, N., Sossalla, S., Ort, K.R., Fluschnik, N., Neumann, K., Seipelt, R., Schondube, F.A., Hasenfuss, G., and Maier, L.S. (2010). CaMKII-dependent diastolic SR Ca2+ leak and elevated diastolic $\mathrm{Ca} 2+$ levels in right atrial myocardium of patients with atrial fibrillation. Circ Res 106, 1134-1144.

Neumann, J., Eschenhagen, T., Jones, L.R., Linck, B., Schmitz, W., Scholz, H., and Zimmermann, N. (1997). Increased expression of cardiac phosphatases in patients with end-stage heart failure. J Mol Cell Cardiol 29, 265-272.

Nichols, C.B., Rossow, C.F., Navedo, M.F., Westenbroek, R.E., Catterall, W.A., Santana, L.F., and McKnight, G.S. (2010). Sympathetic stimulation of adult cardiomyocytes requires association of AKAP5 with a subpopulation of L-type calcium channels. Circ Res 107, 747-756.

Nikolaev, V.O., Bunemann, M., Hein, L., Hannawacker, A., and Lohse, M.J. (2004). Novel single chain cAMP sensors for receptor-induced signal propagation. J Biol Chem 279, 37215-37218.

Nikolaev, V.O., Bunemann, M., Schmitteckert, E., Lohse, M.J., and Engelhardt, S. (2006). Cyclic AMP imaging in adult cardiac myocytes reveals far-reaching beta1-adrenergic but locally confined beta2adrenergic receptor-mediated signaling. Circ Res 99, 1084-1091.

Nikolaev, V.O., Moshkov, A., Lyon, A.R., Miragoli, M., Novak, P., Paur, H., Lohse, M.J., Korchev, Y.E., Harding, S.E., and Gorelik, J. (2010). Beta2-adrenergic receptor redistribution in heart failure changes cAMP compartmentation. Science 327, 1653-1657.

Nony, P., Boissel, J.P., Lievre, M., Leizorovicz, A., Haugh, M.C., Fareh, S., and de Breyne, B. (1994). Evaluation of the effect of phosphodiesterase inhibitors on mortality in chronic heart failure patients. A meta-analysis. Eur J Clin Pharmacol 46, 191-196.

Odermatt, A., Becker, S., Khanna, V.K., Kurzydlowski, K., Leisner, E., Pette, D., and MacLennan, D.H. (1998). Sarcolipin regulates the activity of SERCA1, the fast-twitch skeletal muscle sarcoplasmic reticulum Ca2+-ATPase. J Biol Chem 273, 12360-12369.

Okumura, S., Fujita, T., Cai, W., Jin, M., Namekata, I., Mototani, Y., Jin, H., Ohnuki, Y., Tsuneoka, Y., Kurotani, R., et al. (2014). Epac1-dependent phospholamban phosphorylation mediates the cardiac response to stresses. J Clin Invest 124, 2785-2801.

Osadchii, O.E. (2007). Myocardial phosphodiesterases and regulation of cardiac contractility in health and cardiac disease. Cardiovasc Drugs Ther 21, 171-194.

Packer, M., Carver, J.R., Rodeheffer, R.J., Ivanhoe, R.J., DiBianco, R., Zeldis, S.M., Hendrix, G.H., Bommer, W.J., Elkayam, U., Kukin, M.L., et al. (1991). Effect of oral milrinone on mortality in severe chronic heart failure. The PROMISE Study Research Group. N EngI J Med 325, 1468-1475.

Patrucco, E., Albergine, M.S., Santana, L.F., and Beavo, J.A. (2010). Phosphodiesterase 8A (PDE8A) regulates excitation-contraction coupling in ventricular myocytes. J Mol Cell Cardiol 49, 330-333.

Patterson, A.J., Zhu, W., Chow, A., Agrawal, R., Kosek, J., Xiao, R.P., and Kobilka, B. (2004). Protecting the myocardium: a role for the beta2 adrenergic receptor in the heart. Crit Care Med 32, 1041-1048. Pavlovic, D., Fuller, W., and Shattock, M.J. (2007). The intracellular region of FXYD1 is sufficient to regulate cardiac Na/K ATPase. FASEB J 21, 1539-1546.

Perera, R.K., and Nikolaev, V.O. (2013). Compartmentation of cAMP signalling in cardiomyocytes in health and disease. Acta Physiol (Oxf) 207, 650-662.

Perry, S.J., Baillie, G.S., Kohout, T.A., McPhee, I., Magiera, M.M., Ang, K.L., Miller, W.E., McLean, A.J., Conti, M., Houslay, M.D., et al. (2002). Targeting of cyclic AMP degradation to beta 2-adrenergic receptors by beta-arrestins. Science 298, 834-836.

Ponsioen, B., Zhao, J., Riedl, J., Zwartkruis, F., van der Krogt, G., Zaccolo, M., Moolenaar, W.H., Bos, J.L., and Jalink, K. (2004). Detecting CAMP-induced Epac activation by fluorescence resonance energy transfer: Epac as a novel cAMP indicator. EMBO Rep 5, 1176-1180.

Qian, J., Ren, X., Wang, X., Zhang, P., Jones, W.K., Molkentin, J.D., Fan, G.C., and Kranias, E.G. (2009). Blockade of Hsp20 phosphorylation exacerbates cardiac ischemia/reperfusion injury by suppressed autophagy and increased cell death. Circ Res 105, 1223-1231. 
Qian, J., Vafiadaki, E., Florea, S.M., Singh, V.P., Song, W., Lam, C.K., Wang, Y., Yuan, Q., Pritchard, T.J., Cai, W., et al. (2011). Small heat shock protein 20 interacts with protein phosphatase-1 and enhances sarcoplasmic reticulum calcium cycling. Circ Res 108, 1429-1438.

Reddy, L.G., Jones, L.R., and Thomas, D.D. (1999). Depolymerization of phospholamban in the presence of calcium pump: a fluorescence energy transfer study. Biochemistry 38, 3954-3962.

Richter, W., Day, P., Agrawal, R., Bruss, M.D., Granier, S., Wang, Y.L., Rasmussen, S.G., Horner, K., Wang, P., Lei, T., et al. (2008). Signaling from beta1- and beta2-adrenergic receptors is defined by differential interactions with PDE4. Embo J 27, 384-393.

Richter, W., Xie, M., Scheitrum, C., Krall, J., Movsesian, M.A., and Conti, M. (2011). Conserved expression and functions of PDE4 in rodent and human heart. Basic Res Cardiol 106, 249-262.

Rochais, F., Vandecasteele, G., Lefebvre, F., Lugnier, C., Lum, H., Mazet, J.L., Cooper, D.M., and Fischmeister, R. (2004). Negative feedback exerted by cAMP-dependent protein kinase and cAMP phosphodiesterase on subsarcolemmal cAMP signals in intact cardiac myocytes: an in vivo study using adenovirus-mediated expression of CNG channels. J Biol Chem 279, 52095-52105.

Rosca, M.G., Tandler, B., and Hoppel, C.L. (2013). Mitochondria in cardiac hypertrophy and heart failure. J Mol Cell Cardiol 55, 31-41.

Sample, V., DiPilato, L.M., Yang, J.H., Ni, Q., Saucerman, J.J., and Zhang, J. (2012). Regulation of nuclear PKA revealed by spatiotemporal manipulation of cyclic AMP. Nat Chem Biol 8, 375-382.

Sande, J.B., Sjaastad, I., Hoen, I.B., Bokenes, J., Tonnessen, T., Holt, E., Lunde, P.K., and Christensen, G. (2002). Reduced level of serine(16) phosphorylated phospholamban in the failing rat myocardium: a major contributor to reduced SERCA2 activity. Cardiovasc Res 53, 382-391.

Schlotthauer, K., and Bers, D.M. (2000). Sarcoplasmic reticulum $\mathrm{Ca}(2+)$ release causes myocyte depolarization. Underlying mechanism and threshold for triggered action potentials. Circ Res 87, 774780 .

Scholten, A., van Veen, T.A., Vos, M.A., and Heck, A.J. (2007). Diversity of cAMP-dependent protein kinase isoforms and their anchoring proteins in mouse ventricular tissue. J Proteome Res 6, 17051717.

Schwinger, R.H., Munch, G., Bolck, B., Karczewski, P., Krause, E.G., and Erdmann, E. (1999). Reduced $\mathrm{Ca}(2+)$-sensitivity of SERCA $2 \mathrm{a}$ in failing human myocardium due to reduced serin-16 phospholamban phosphorylation. J Mol Cell Cardiol 31, 479-491.

Scott, J.D., Dessauer, C.W., and Tasken, K. (2013). Creating order from chaos: cellular regulation by kinase anchoring. Annu Rev Pharmacol Toxicol 53, 187-210.

Scott, J.D., and Santana, L.F. (2010). A-kinase anchoring proteins: getting to the heart of the matter. Circulation 121, 1264-1271.

Shan, J., Betzenhauser, M.J., Kushnir, A., Reiken, S., Meli, A.C., Wronska, A., Dura, M., Chen, B.X., and Marks, A.R. (2010). Role of chronic ryanodine receptor phosphorylation in heart failure and betaadrenergic receptor blockade in mice. J Clin Invest 120, 4375-4387.

Silverman, B., Fuller, W., Eaton, P., Deng, J., Moorman, J.R., Cheung, J.Y., James, A.F., and Shattock, M.J. (2005). Serine 68 phosphorylation of phospholemman: acute isoform-specific activation of cardiac Na/K ATPase. Cardiovasc Res 65, 93-103.

Simmerman, H.K., Collins, J.H., Theibert, J.L., Wegener, A.D., and Jones, L.R. (1986). Sequence analysis of phospholamban. Identification of phosphorylation sites and two major structural domains. J Biol Chem 261, 13333-13341.

Sin, Y.Y., Edwards, H.V., Li, X., Day, J.P., Christian, F., Dunlop, A.J., Adams, D.R., Zaccolo, M., Houslay, M.D., and Baillie, G.S. (2011). Disruption of the cyclic AMP phosphodiesterase-4 (PDE4)-HSP20 complex attenuates the beta-agonist induced hypertrophic response in cardiac myocytes. J Mol Cell Cardiol 50, 872-883.

Singh, A., Redden, J.M., Kapiloff, M.S., and Dodge-Kafka, K.L. (2011). The large isoforms of A-kinase anchoring protein 18 mediate the phosphorylation of inhibitor-1 by protein kinase $A$ and the inhibition of protein phosphatase 1 activity. Mol Pharmacol 79, 533-540.

Soderling, S.H., Bayuga, S.J., and Beavo, J.A. (1998). Cloning and characterization of a cAMP-specific cyclic nucleotide phosphodiesterase. Proc Natl Acad Sci U S A 95, 8991-8996. 
Sonnenburg, W.K., Seger, D., and Beavo, J.A. (1993). Molecular cloning of a cDNA encoding the "61$\mathrm{kDa}$ " calmodulin-stimulated cyclic nucleotide phosphodiesterase. Tissue-specific expression of structurally related isoforms. J Biol Chem 268, 645-652.

Sprenger, J.U., and Nikolaev, V.O. (2013). Biophysical Techniques for Detection of cAMP and cGMP in Living Cells. Int J Mol Sci 14, 8025-8046.

Sprenger, J.U., Perera, R.K., Gotz, K.R., and Nikolaev, V.O. FRET microscopy for real-time monitoring of signaling events in live cells using unimolecular biosensors. J Vis Exp, e4081.

Sprenger, J.U., Perera, R.K., Gotz, K.R., and Nikolaev, V.O. (2012). FRET microscopy for real-time monitoring of signaling events in live cells using unimolecular biosensors. J Vis Exp, e4081.

Stangherlin, A., Gesellchen, F., Zoccarato, A., Terrin, A., Fields, L.A., Berrera, M., Surdo, N.C., Craig, M.A., Smith, G., Hamilton, G., et al. (2011). cGMP signals modulate cAMP levels in a compartmentspecific manner to regulate catecholamine-dependent signaling in cardiac myocytes. Circ Res 108, 929-939.

Steenaart, N.A., Ganim, J.R., Di Salvo, J., and Kranias, E.G. (1992). The phospholamban phosphatase associated with cardiac sarcoplasmic reticulum is a type 1 enzyme. Arch Biochem Biophys 293, 17-24. Strang, K.T., Sweitzer, N.K., Greaser, M.L., and Moss, R.L. (1994). Beta-adrenergic receptor stimulation increases unloaded shortening velocity of skinned single ventricular myocytes from rats. Circ Res 74, 542-549.

Tada, M., and Katz, A.M. (1982). Phosphorylation of the sarcoplasmic reticulum and sarcolemma. Annu Rev Physiol 44, 401-423.

Takasago, T., Imagawa, T., and Shigekawa, M. (1989). Phosphorylation of the cardiac ryanodine receptor by cAMP-dependent protein kinase. J Biochem 106, 872-877.

Tasken, K., and Aandahl, E.M. (2004). Localized effects of cAMP mediated by distinct routes of protein kinase A. Physiol Rev 84, 137-167.

Taylor, S.S., Buechler, J.A., and Yonemoto, W. (1990). cAMP-dependent protein kinase: framework for a diverse family of regulatory enzymes. Annu Rev Biochem 59, 971-1005.

Taylor, S.S., Kim, C., Cheng, C.Y., Brown, S.H., Wu, J., and Kannan, N. (2008). Signaling through cAMP and cAMP-dependent protein kinase: diverse strategies for drug design. Biochim Biophys Acta 1784, 16-26.

Tengholm, A., and Gylfe, E. (2009). Oscillatory control of insulin secretion. Mol Cell Endocrinol 297, 58-72.

Terrin, A., Di Benedetto, G., Pertegato, V., Cheung, Y.F., Baillie, G., Lynch, M.J., Elvassore, N., Prinz, A., Herberg, F.W., Houslay, M.D., et al. (2006). PGE(1) stimulation of HEK293 cells generates multiple contiguous domains with different [cAMP]: role of compartmentalized phosphodiesterases. J Cell Biol 175, 441-451.

Thompson, W.J., and Appleman, M.M. (1971). Multiple cyclic nucleotide phosphodiesterase activities from rat brain. Biochemistry 10, 311-316.

Torgersen, K.M., Vang, T., Abrahamsen, H., Yaqub, S., and Tasken, K. (2002). Molecular mechanisms for protein kinase A-mediated modulation of immune function. Cell Signal 14, 1-9.

Towbin, H., Staehelin, T., and Gordon, J. (1979). Electrophoretic transfer of proteins from polyacrylamide gels to nitrocellulose sheets: procedure and some applications. Proc Natl Acad Sci U S A 76, 4350-4354.

Troger, J., Moutty, M.C., Skroblin, P., and Klussmann, E. (2012). A-kinase anchoring proteins as potential drug targets. Br J Pharmacol 166, 420-433.

Tsien, R.Y. (1998). The green fluorescent protein. Annu Rev Biochem 67, 509-544.

Ungerer, M., Bohm, M., Elce, J.S., Erdmann, E., and Lohse, M.J. (1993). Altered expression of betaadrenergic receptor kinase and beta 1-adrenergic receptors in the failing human heart. Circulation 87, 454-463.

Vafiadaki, E., Arvanitis, D.A., Pagakis, S.N., Papalouka, V., Sanoudou, D., KontrogianniKonstantopoulos, A., and Kranias, E.G. (2009). The anti-apoptotic protein HAX-1 interacts with SERCA2 and regulates its protein levels to promote cell survival. Mol Biol Cell 20, 306-318. 
Vafiadaki, E., Sanoudou, D., Arvanitis, D.A., Catino, D.H., Kranias, E.G., and KontrogianniKonstantopoulos, A. (2007). Phospholamban interacts with HAX-1, a mitochondrial protein with antiapoptotic function. J Mol Biol 367, 65-79.

Vila Petroff, M.G., Egan, J.M., Wang, X., and Sollott, S.J. (2001). Glucagon-like peptide-1 increases cAMP but fails to augment contraction in adult rat cardiac myocytes. Circ Res 89, 445-452.

Violin, J.D., DiPilato, L.M., Yildirim, N., Elston, T.C., Zhang, J., and Lefkowitz, R.J. (2008). beta2adrenergic receptor signaling and desensitization elucidated by quantitative modeling of real time cAMP dynamics. J Biol Chem 283, 2949-2961.

Vittone, L., Mundina, C., Chiappe de Cingolani, G., and Mattiazzi, A. (1990). cAMP and calciumdependent mechanisms of phospholamban phosphorylation in intact hearts. Am J Physiol 258, H318325.

Warrier, S., Belevych, A.E., Ruse, M., Eckert, R.L., Zaccolo, M., Pozzan, T., and Harvey, R.D. (2005). Beta-adrenergic- and muscarinic receptor-induced changes in CAMP activity in adult cardiac myocytes detected with FRET-based biosensor. Am J Physiol Cell Physiol 289, C455-461.

Wehrens, X.H., Lehnart, S.E., Huang, F., Vest, J.A., Reiken, S.R., Mohler, P.J., Sun, J., Guatimosim, S., Song, L.S., Rosemblit, N., et al. (2003). FKBP12.6 deficiency and defective calcium release channel (ryanodine receptor) function linked to exercise-induced sudden cardiac death. Cell 113, 829-840.

Weishaar, R.E., Kobylarz-Singer, D.C., Steffen, R.P., and Kaplan, H.R. (1987). Subclasses of cyclic AMPspecific phosphodiesterase in left ventricular muscle and their involvement in regulating myocardial contractility. Circ Res 61, 539-547.

Wikman-Coffelt, J., Sievers, R., Coffelt, R.J., and Parmley, W.W. (1983). Oscillations of cAMP with the cardiac cycle. Biochem Biophys Res Commun 111, 450-455.

Williams, C. (2004). cAMP detection methods in HTS: selecting the best from the rest. Nat Rev Drug Discov 3, 125-135.

Willoughby, D., and Cooper, D.M. (2007). Organization and Ca2+ regulation of adenylyl cyclases in cAMP microdomains. Physiol Rev 87, 965-1010.

Wong, W., and Scott, J.D. (2004). AKAP signalling complexes: focal points in space and time. Nat Rev Mol Cell Biol 5, 959-970.

Wu, P., and Brand, L. (1994). Resonance energy transfer: methods and applications. Anal Biochem 218, 1-13.

Xiao, R.P. (2001). Beta-adrenergic signaling in the heart: dual coupling of the beta2-adrenergic receptor to G(s) and G(i) proteins. Sci STKE 2001, re15.

Zaccolo, M. (2009). cAMP signal transduction in the heart: understanding spatial control for the development of novel therapeutic strategies. Br J Pharmacol 158, 50-60.

Zaccolo, M., De Giorgi, F., Cho, C.Y., Feng, L., Knapp, T., Negulescu, P.A., Taylor, S.S., Tsien, R.Y., and Pozzan, T. (2000). A genetically encoded, fluorescent indicator for cyclic AMP in living cells. Nat Cell Biol 2, 25-29.

Zaccolo, M., and Movsesian, M.A. (2007). cAMP and cGMP signaling cross-talk: role of phosphodiesterases and implications for cardiac pathophysiology. Circ Res 100, 1569-1578.

Zaccolo, M., and Pozzan, T. (2002). Discrete microdomains with high concentration of cAMP in stimulated rat neonatal cardiac myocytes. Science 295, 1711-1715.

Zagotta, W.N., Olivier, N.B., Black, K.D., Young, E.C., Olson, R., and Gouaux, E. (2003). Structural basis for modulation and agonist specificity of HCN pacemaker channels. Nature 425, 200-205.

Zakhary, D.R., Fink, M.A., Ruehr, M.L., and Bond, M. (2000a). Selectivity and regulation of A-kinase anchoring proteins in the heart. The role of autophosphorylation of the type II regulatory subunit of cAMP-dependent protein kinase. J Biol Chem 275, 41389-41395.

Zakhary, D.R., Moravec, C.S., and Bond, M. (2000b). Regulation of PKA binding to AKAPs in the heart: alterations in human heart failure. Circulation 101, 1459-1464.

Zamoon, J., Mascioni, A., Thomas, D.D., and Veglia, G. (2003). NMR solution structure and topological orientation of monomeric phospholamban in dodecylphosphocholine micelles. Biophys J 85, 25892598. 
Zhai, J., Schmidt, A.G., Hoit, B.D., Kimura, Y., MacLennan, D.H., and Kranias, E.G. (2000). Cardiacspecific overexpression of a superinhibitory pentameric phospholamban mutant enhances inhibition of cardiac function in vivo. J Biol Chem 275, 10538-10544.

Zhang, J., Hupfeld, C.J., Taylor, S.S., Olefsky, J.M., and Tsien, R.Y. (2005). Insulin disrupts betaadrenergic signalling to protein kinase A in adipocytes. Nature 437, 569-573.

Zhang, J., Ma, Y., Taylor, S.S., and Tsien, R.Y. (2001). Genetically encoded reporters of protein kinase A activity reveal impact of substrate tethering. Proc Natl Acad Sci U S A 98, 14997-15002.

Zhao, W., Waggoner, J.R., Zhang, Z.G., Lam, C.K., Han, P., Qian, J., Schroder, P.M., Mitton, B., Kontrogianni-Konstantopoulos, A., Robia, S.L., et al. (2009). The anti-apoptotic protein HAX-1 is a regulator of cardiac function. Proc Natl Acad Sci U S A 106, 20776-20781.

Zvaritch, E., Backx, P.H., Jirik, F., Kimura, Y., de Leon, S., Schmidt, A.G., Hoit, B.D., Lester, J.W., Kranias, E.G., and MacLennan, D.H. (2000). The transgenic expression of highly inhibitory monomeric forms of phospholamban in mouse heart impairs cardiac contractility. J Biol Chem 275, 14985-14991. 


\section{Acknowledgements}

I would like to thank my primary supervisor Dr. Viacheslav Nikolaev for giving me the opportunity to work on this interesting and exciting research topic. He provided me with great support and advice throughout my project.

Besides, I would like to thank the other members of my thesis committee, Dr. Sebastian Kügler and Prof. Dr. Peter Schu, for their challenging ideas and fruitful discussions during the progress report meetings.

A special thank goes to Tobias Goldak and Karina Zimmermann for excellent technical assistance, especially Karina Zimmermann, who always supported me in the lab with her expertise regarding immunoblot and immunohistochemistry procedures. Besides, I would like to thank all members of the "Nikolaev lab" who were always helpful when I had to deal with unexpected problems and kept my spirits up.

My sincere thanks go to Julia Steinbrecher, who performed the TAC and sham surgeries so conscientiously and accurately, that she provided an essential basis for my PhD project.

I would like to thank the SFB service unit including Roland Blume, Marcel Zorembra and Beate Knocke as well as Kirsten Koschel and Sabrina Wollborn for their support regarding echocardiography performance and data analysis.

Thanks to Mei Ling Chang Liao for her technical advice on the lonOptix setup which often challenged us with new adjustment problems.

I would also like to thank the members from the group around Prof. Dr. med. Lars Meier, particularly Felicia Steuer and Thomas Sowa, for calcium measurements and advice on immunoblot optimizing, respectively.

Besides, I thank Prof. Stephan Lehnart for his thoughtful comments on my manuscript, which is now under revision.

Sincere thanks to my family for the financial and especially mental support during the four years of my Phd thesis.

My special appreciation goes to Ruwan Perera for his love and support during the highs and lows that were linked to the last four years. Thank you. 